\title{
Internal kinematics of spiral galaxies in distant clusters
}

\section{Velocity fields from FORS2/MXU spectroscopy $\star, \star \star$}

\author{
E. Kutdemir ${ }^{1,2}$, B. L. Ziegler ${ }^{3,1}$, R. F. Peletier ${ }^{2}$, C. Da Rocha ${ }^{3,1}$, T. Kronberger ${ }^{4}$, W. Kapferer ${ }^{4}$, \\ S. Schindler ${ }^{4}$, A. Böhm ${ }^{5}$, K. Jäger ${ }^{6}$, H. Kuntschner ${ }^{7}$, and M. Verdugo ${ }^{1}$ \\ ${ }^{1}$ Institut für Astrophysik, Georg-August-Universität, Friedrich-Hund-Platz 1, 37077 Göttingen, Germany \\ e-mail: kutdemir@astro.rug.nl \\ 2 Kapteyn Astronomical Institute, PO BOX 800, 9700 AV Groningen, The Netherlands \\ 3 European-Southern Observatory, Karl-Schwarzschild Str. 2, 85748 Garching, Germany \\ ${ }^{4}$ Institut für Astro- und Teilchenphysik, Universität Innsbruck, Technikerstrasse 25, 6020 Innsbruck, Austria \\ 5 Astrophysikalisches Institut Potsdam, An der Sternwarte 16, 14482 Potsdam, Germany \\ ${ }^{6}$ Max-Planck-Institut für Astronomie, 69117 Heidelberg, Germany \\ 7 ST-ECF, Karl-Schwarzschild Str. 2, 85748 Garching, Germany
}

Received 14 March 2008 / Accepted 20 June 2008

\begin{abstract}
Context. We continue our investigation on how the cluster environment affects the evolution of galaxies.

Aims. By examining both galaxy structure and internal kinematics of cluster galaxies at lookback times of $\sim 5$ Gyr we study the nature and impact of possible interactions at the peak epoch of cluster assembly.

Methods. Going beyond our previous measurements of two-dimensional rotation curves, we here observe the whole velocity field of the galaxies of the sample. We achieve a complete coverage and optimal spatial sampling of galaxy sizes by placing three adjacent and parallel FORS2 MXU (Mask eXchange Unit) slits onto each object yielding simultaneously several emission and absorption lines. We reconstruct the gas velocity field and decompose it into circular rotation and irregular motions using a harmonic decomposition method called kinemetry. To measure the irregularity in the gas kinematics, we define 3 parameters: $\sigma_{\mathrm{PA}}$ (the standard deviation of the kinematic position angle within a galaxy), $\Delta \phi$ (the average misalignment between kinematic and photometric position angles) and $k_{3,5}$ (squared sum of the higher order Fourier terms).

Results. We present the analysis of the velocity fields and morphology of 22 distant galaxies in the MS 0451.6-0305 field with 11 members at $z=0.54$ and a local sample from SINGS. Using local, undistorted galaxies the three parameters $\sigma_{\mathrm{PA}}, \Delta \phi$ and $k_{3,5}$ can be used to establish the regularity of the gas velocity fields. Among the galaxies for which we could measure these parameters, we find both field ones ( 4 of 8 ) and cluster members ( 3 of 4 ), which have a velocity field that we consider both irregular and asymmetric. We show that these fractions are underestimates of the total number of objects with irregular velocity fields. The values of the irregularity parameters for cluster galaxies are not very different from those of the field galaxies, implying that there are isolated field galaxies that are as distorted as the cluster members. None of the deviations in our small sample correlate with photometric/structural properties like luminosity or disk scale length in a significant way.

Conclusions. We have demonstrated that our 3D-spectroscopic method successfully maps the velocity field of distant galaxies. Together with a structural analysis the importance and efficiency of cluster specific interactions can be assessed quantitatively.
\end{abstract}

Key words. galaxies: evolution - galaxies: kinematics and dynamics - galaxies: clusters: individual: MS 0451.6-0305 galaxies: spiral

\section{Introduction}

The motion of stars and gas clouds within a galaxy are important measurable characteristics representative of the whole system. Since the internal kinematics are subject to the overall gravitational potential they provide a proxy for the total mass. In addition to the baryonic mass of gas and stars, that may be inferred from photometric observations, velocities trace the dark

\footnotetext{
* Based on observations collected at the European Southern Observatory (ESO), Cerro Paranal, Chile (ESO Nos. 66.A-0546, 66.A-0547 \& 74.B-0592).

$\star \star$ Appendices $\mathrm{A}-\mathrm{C}$ are only available in electronic form at http://www . aanda.org
}

matter distribution (e.g. Sofue \& Rubin 2001). The (ir)regularity of its 3-dimensional velocity field can provide clues about possible distortions of a galaxy, such as warps. Peculiar velocity fields may also be indicators of recent or ongoing interaction processes. This is particularly important in the environment of galaxy clusters, where specific interactions occur in addition to merging and accretion events that are observed in the field population and that are elements of the hierarchical growth of structure in the Universe (e.g. van Gorkom 2004; Poggianti 2004). Cluster specific processes are presumably rather frequent at redshifts $z \approx 0.4-1$, because the assembly of galaxy clusters is expected to peak at these epochs under the conditions of the concordance cosmology (e.g. Bower 1991; Kay et al. 2007). That is 
also the reason why fundamental properties of galaxies at these redshifts are measured and compared to those of local objects in order to explore galaxy formation and evolution.

There are considerable advantages in using 3-dimensional spectroscopy over conventional long-slit data. Apart from a better assessment of the interaction origin of galaxies it also improves the accuracy of the establishment of scaling relations. For example, an important tool to measure the cosmological evolution of disk galaxies is the Tully-Fisher relation (TFR, Tully \& Fisher 1977) where the parameter next to the intrinsic luminosity is the maximum velocity $V_{\max }$ of the flat part of the rotation curve. Only for very regular Rotation Curves (RC's), where the turn-over and the flat part are clearly visible, $V_{\max }$ can be derived with sufficient accuracy to include the galaxy under scrutiny into a TF analysis. This is particularly important in the case of distant, small and faint galaxies (e.g. Ziegler et al. 2002; Böhm et al. 2004; Conselice et al. 2005; Bamford et al. 2006; Kassin et al. 2007; Böhm \& Ziegler 2007). The assessment of RC quality may also be the main reason why recent studies of the cluster TFR evolution differ from each other with respect to sign and amount of offset between distant galaxies in the cluster and the field environment (Ziegler et al. 2003; Bamford et al. 2005; Nakamura et al. 2005; Metevier et al. 2006).

Apparently smooth RCs can nevertheless result in wrong estimates of $V_{\max }$, if for example the position angle of the major axis, as measured from the photometry, is not the same as the kinematic position angle. The same holds if the photometric center does not agree with the center measured from the kinematics. A way to resolve the situation is to obtain 3-dimensional kinematical information. For example, Mendes de Oliveira et al. (2003) use velocity fields of galaxies in compact groups obtained using Fabry-Pérot spectroscopy to show that smooth RCs can be derived for most galaxies that were previously judged to be distorted on the basis of the limited information given by 2D-spectra (Rubin et al. 1991). Alternatively, in some cases the rotation curve along the photometric axis may look regular even though the velocity field is distorted. Because of this, 3-dimensional information is clearly preferable to long-slit data. It remains a requirement that the observed velocity fields should cover the flat part of the rotation curve.

Several comprehensive studies of galaxies in the Local Universe exist that explore velocity fields using optical observations. Fabry-Pérot interferometry of the $\mathrm{H} \alpha$ emission line is the basis of the GHASP (Garrido et al. 2005), SINGS (Daigle et al. 2006) and Virgo (Chemin et al. 2006) surveys, for example. 3D-spectroscopy is regularly performed with the SAURON integral field unit, delivering spectra within a limited wavelength range, with which stellar absorption lines can be investigated in addition to gaseous emission lines (e.g. Ganda et al. 2006; Falcón-Barroso et al. 2006; Sarzi et al. 2006). Integral-field spectroscopy of HII regions in nearby disk galaxies was established, as another example, with DensePak on the WIYN $3.5 \mathrm{~m}$ telescope (Andersen et al. 2006). While spatially resolved H I measurements with radio telescopes are quite common locally, (e.g. Boomsma et al. 2005; Noordermeer et al. 2007) such observations at higher redshift are just becoming feasible with new instrumentation (e.g. EVLA, APEX).

At $z \gtrsim 0.2$, studies of the global velocity field of galaxies in the optical and near-infrared regimes are also quite challenging. While in the NIR a high spatial resolution can be achieved thanks to the combination of 3D-spectroscopy with adaptiveoptics techniques (Förster Schreiber et al. 2006), one is hampered by seeing effects in the optical (Kronberger et al. 2007). One of the more comprehensive optical studies has made use of the 15 deployable small IFUs (integral field units) of the FLAMES instrument at the VLT. That way, 35 field galaxies at $0.4<z<0.7$ were examined and analyzed using the TFR (Flores et al. 2006). Eliminating the galaxies that have perturbed or complex kinematics, they found no evolution in the Tully Fisher Relation since $z=0.6$. Other investigations are restricted to a small number of objects. Using the GMOS integral field spectrograph and exploiting the light magnification of a foreground cluster, Swinbank et al. (2006) for example, probed the emission-line properties of six $z=1$ field galaxies in the background. Investigating where these galaxies lie on the $B$ and $I$ band TFR compared to local galaxies, they reported that in the $B$-band the galaxies are brightened by $0.5 \pm 0.3 \mathrm{mag}$, while in the $I$ band they are in agreement with the local relation.

To determine the nature and efficiency of interaction processes in the cluster environment we continue our study of galaxies at $z \approx 0.5$ with new spectroscopy using FORS2 at the VLT and HST/ACS imaging. Our project involves observations of four different cluster fields (MS 0451.6-0305 at $z=0.54$, MS 1008.1-1224 at $z=0.301, \mathrm{~F} 1557.19 \mathrm{TC}$ at $z=0.510$, MS 2137.3-2353 at $z=0.313$ ) with about 20 galaxies in each of them. While in our previous campaigns we derived the internal kinematics along the photometric major axis of the galaxies (Ziegler et al. 2003; Jäger et al. 2004) this time we measure the three-dimensional velocity fields of the galaxies. We achieve this by placing three adjacent, parallel FORS2 MXU (Mask eXchange Unit) slits onto the same galaxy. In HST Cycle 14 the clusters were observed using the ACS camera (PID 10635), covering the full field-of-view of the spectroscopy by a $2 \times 2$ mosaic allowing a detailed morphological and structural analysis of all target galaxies. In this paper, we present the results of our kinematic and photometric analysis of the cluster MS 0451 at $z=0.54$ (Donahue 1996), classify the galaxies according to the regularity of their gas kinematics and investigate whether this is related to the environment (cluster/field) and some other properties like galaxy type, luminosity, disk scale length, etc. In followup papers, similar data for the other three clusters will be investigated. In addition, we will compare the kinematic and structural analysis with hydrodynamic $N$-body simulations of both isolated (Kapferer et al. 2006) and interacting galaxies (Kronberger et al. 2006). Using these simulations we already examined systematically what is the effect of observational constraints like a low spatial sampling in the case of distant small galaxies on the measured velocity fields (Kronberger et al. 2007). In a final paper we will combine all our results to address the question of the interaction history of cluster galaxies.

In Sect. 2, we outline our unique approach of "matched IFU simulations" using MXU masks with the FORS2 spectrograph at the VLT and give an account of our spectral reduction, explain how the velocity fields are constructed and describe the observed sample in the field of MS 0451. Our kinematic analysis of the gas velocity fields and how we measure the stellar kinematics are also explained in this section. In Sect. 3, we describe how we derive structural parameters from HST images and luminosities in different filters from ground-based photometry. In Sect. 4, we explain how we quantify the deviations from regularity in the gas kinematics, present the results of that analysis, make a comparison between the field and cluster galaxies and investigate the existence of a relation between the kinematic regularity and some photometric properties. In Sect. 5, we discuss our results. In Sect. 6 we summarize the paper and draw our conclusions. In Appendix A, we discuss the effects of the spatial resolution on our kinematical analysis. Kinematic and photometric information on individual galaxies in the MS 0451 cluster 
field are given in Appendix B. Throughout this paper we use a standard cosmology with: $H_{0}=70 \mathrm{~km} \mathrm{~s}^{-1} \mathrm{Mpc}^{-1}, \Omega_{\mathrm{m}}=0.3$, $\Omega_{\lambda}=0.7$ (Tonry et al. 2003).

\section{3D-spectroscopy with FORS2/MXU}

\subsection{Observing strategy}

Our aim was to obtain spatially resolved emission line rotation curves for galaxies with typical surface brightnesses of $V \approx 22.5 \mathrm{mag} / /^{\prime \prime 2}$. This is feasible in a reasonable time only with large ground based telescopes. The natural approach to observe 3D-velocity fields would be to use IFUs to achieve some kind of 3D-spectroscopy. Inspecting the performance of all such instruments available at the ESO-VLT, it turned out that none of them are optimally suited and efficient for our purpose. One of the main goals of our study, although not of the current paper, is to unambiguously determine the maximum rotation velocity $V_{\max }$ of galaxies with regular velocity fields. From our previous campaigns, we knew that many spirals at $z=0.3-0.6$ have rather large sizes with the turn-over points of their RCs at radii around $1^{\prime \prime} .5-2^{\prime \prime}$, corresponding to disk scale lengths of $\approx 3 R_{\mathrm{d}}$ on either side of the center of the galaxies (see Ziegler et al. 2003, Fig. 1). Thus, the FLAMES/ARGUS field-of-view in high-resolution mode $\left(4^{\prime \prime} .2 \times 66^{\prime \prime} 6\right)$ would just have been adequate, but given the low throughput of the high-resolution spectrograph GIRAFFE, the necessary exposure times would have been so high that even a full night would not have been sufficient to observe just one galaxy. With the VIMOS IFU, still only 2 or 3 objects could have been observed per night due to the small field of view. Suitable to some extent are only the 15 deployable IFUs of FLAMES, whose octagonal lens array has a spatial coverage of $\approx 2^{\prime \prime} \times 3^{\prime \prime}$. This does not probe the outer regions of rotation curves, which is vital for an accurate determination of $V_{\max }$. Only extrapolation of the measurements from the inner parts of the VF gives an estimate of $V_{\max }$ (see, e.g., Fig. 7 in Flores et al. 2006). No real way out is the possibility to use a mosaic of four pointings per field, because then the necessary observing time would be too large.

For this reason and for other reasons stated below, we conceived a method to "simulate IFUs" that are exactly matched to our purpose using the FORS2 focal reducer spectrograph. This instrument offers a mode (MXU) using custom-made slit masks, in which slitlets can be rotated with respect to the y-axis of the CCD (Schink et al. 2000). We designed three masks for each cluster field, so that each object was subsequently covered by three different, but parallel slits of $1^{\prime \prime}$ width. One slit was placed along the photometric major axis of a galaxy, the other two were shifted by $1^{\prime \prime}$ along the minor axis on both sides of the central slit, respectively (Fig. 1). This resulted in an appropriate aperture of $\sim 7^{\prime \prime} \times 3^{\prime \prime}$ for each target (the actual dimensions of the slits along the major axes are much longer to retrieve the velocities at larger distances from the center than $33^{\prime \prime} 5$, in case there is still signal, and to allow accurate sky subtraction). Although each cluster field must be observed three times (with three different masks), this method is still very efficient due to two reasons. First, the high-throughput VPH grisms of FORS2 provide the necessary $S / N$ in the spectra with an integration time of $2.5 \mathrm{~h}$ only, i.e. for all three setups together $7.5 \mathrm{~h}$ would be needed (comparable/lower than shutter-open times needed with FLAMES). Second, the multiplex capability of designing many slits across the full field of FORS2 $\left(6^{\prime} \times 6^{\prime}\right)$ allows to observe simultaneously a rather high number of objects (in case of MS 0451 there were 20 slits for 22 galaxies).
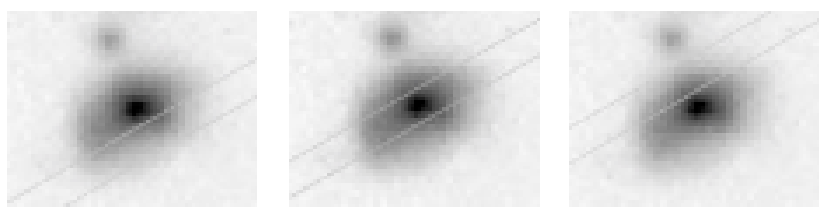

Fig. 1. Example of the positioning of three slits onto one galaxy (galaxy C8).

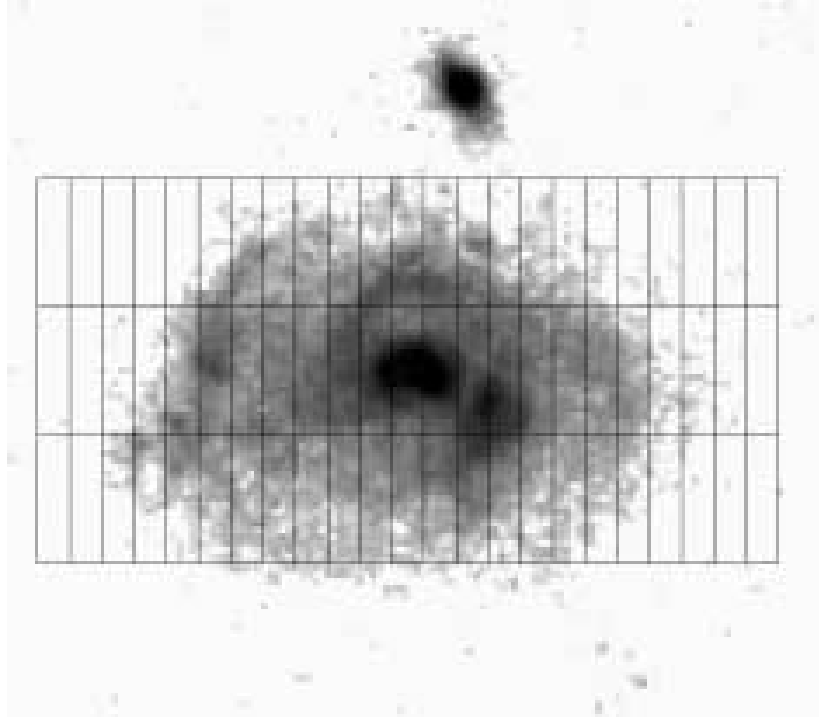

Fig. 2. HST/ACS image of a member galaxy (galaxy C $8, z=0.5326$ ) of cluster MS 0451 . The overlaid grid indicates the 3 slit positions with each slit ( $1^{\prime \prime}$ width) subdivided into FORS pixels $(0$ '.25) that correspond to spectral rows.

Our approach has several additional advantages: since the pixel scale of the two FORS2 MIT-CCD chips is $0.25 /$ pixel, our spatial resolution of the resulting velocity fields is very adequate for the investigated redshifts (Fig. 2) and is in practise only limited by the seeing. An adequate spectral resolution of $R \approx 1000$ is achieved by using the holographic grism grism_600RI. The instrumental resolution we measure from the data is $5.6 \AA(F W H M)$ which corresponds to $\sigma_{\text {inst }} \sim 120 \mathrm{~km} \mathrm{~s}^{-1}$ at $6000 \AA$. Here we are able to measure the velocities down to $\sim \sigma / 10 \mathrm{~km} \mathrm{~s}^{-1}$, i.e. the typical accuracy of our velocities is $\sim 12 \mathrm{~km} \mathrm{~s}^{-1}$. With this grism a rather large wavelength coverage was obtained, making it possible to simultaneously observe several emission lines, allowing independent determinations of the velocity field (VF), as well as many absorption lines, that can be used to derive, for example, stellar rotation curves (see Sect. 2.5). For the cluster members of MS 0451, we probe the emission lines of $[\mathrm{O}$ II $] 3727, \mathrm{H} \gamma, \mathrm{H} \beta,[\mathrm{O} \mathrm{III}] 4959$ and [O III]5007. Line ratios can then be used, for example, to derive the gas-phase metallicities of the galaxies or to detect possible contaminations from AGN. To summarize, our observational strategy provides a matched aperture size, large target coverage and a wide range of wavelength for efficiently low exposure times. Spatial sampling is sufficient $(0.25)$ along the spatial axis while it is $1^{\prime \prime}$ along the spectral axis (Fig. 2).

\subsection{Data reduction}

Each cluster field was observed with three separate masks to be able to place three parallel slits onto each galaxy; the central one along the photometric major axis and the other two $1^{\prime \prime}$ offset 
along the minor axis in opposite directions. For cosmic ray removal, the integration time for each mask was split into three exposures. The observations for MS 0451 were spread across 6 nights in January and February 2005. In the case of one mask, all exposures were taken during the same night. For that mask, all science frames were combined right after the overscan and bias correction process, since the alignment of the frames was excellent. For the rest, the order of the reduction steps was the following:

- overscan and bias correction of all science and calibration frames;

- extracting 2D spectra of all objects, corresponding arc spectra and flat fields from the main frames;

- normalization of each flat field frame;

- flat field correction of each science and arc spectrum;

- wavelength calibration using the arc lamp spectra first and then using around 10 strong sky lines to correct for the shift caused by the movement of the telescope during the observation;

- sky subtraction;

- rebinning the spectra to ensure that all the spectra start at the same wavelength and have the same wavelength interval per pixel;

- checking the alignment of the spectra taken with the same mask (the difference between the photometric center at a certain wavelength in 3 different exposures for each galaxy spectrum was on the subpixel level, so no shift had to be applied before combining them);

- median combining the three exposures for each mask;

- correcting for the curvature of the spectrum caused by the optics of the instrument. After summing up each column with around 100 surrounding columns, a parabola was fitted to the positions of maxima at each column to trace the curvature.

\subsection{Galaxies in the MS 0451 sample}

In this paper, we present the analysis of the cluster field of MS 0451 at $z=0.54$. Twenty-six galaxies in this cluster had already been spectroscopic targets in our previous campaigns, similar to the MOS observations described by Ziegler et al. (2003) and Jäger et al. (2004). In these previous runs, only one MXU slit was placed onto each galaxy, mostly along the photometric major axis as determined from (ground-based) FORS images (ESO PID 66.A-0547). Due to geometric constraints (mask setup) or when two objects were forced into the same slit other slit orientations were chosen. The results from this campaign will be presented in another paper of this series. Examples of four galaxies have already been given in Kronberger et al. (2006).

Since for these galaxies accurate redshifts and equivalent widths of visible emission lines were available, they formed the primary candidate list for target selection for our new study presented here. First priority was given to cluster members with significant emission in [OII]3727, second priority to field galaxies with a clear detection of any emission line that would fall onto our chosen observed wavelength range. Note that no morphological information was used for the sample selection. Further objects were drawn from a catalog provided by the CNOC survey (Ellingson et al. 1998) with either redshift information or measured $(g-r)$ color that matches expectations for spiral templates at $z \approx 0.5$. If there was space in the MXU setup, and no suitable candidate at all was available, a galaxy was picked at random. In
Table 1. Basic galaxy information.

\begin{tabular}{|c|c|c|c|c|c|}
\hline $\begin{array}{l}\text { ID } \\
\text { (1) }\end{array}$ & $\begin{array}{c}z \\
(2)\end{array}$ & $\begin{array}{l}d \\
\text { (3) }\end{array}$ & $\begin{array}{l}\text { NED name } \\
\text { (4) }\end{array}$ & $\begin{array}{l}\text { Type, Ref. } \\
\text { (5) }\end{array}$ & $\begin{array}{l}z, \text { Ref. } \\
\text { (6) }\end{array}$ \\
\hline \multirow[t]{2}{*}{$\mathrm{C} 1$} & 0.5421 & 1.7 & PPP 002414 & Sb-Sc (1) & $0.54160(1)$ \\
\hline & & & & $\mathrm{Sab}(2)$ & $0.54240(2)$ \\
\hline $\mathrm{C} 2$ & 0.5486 & 0.7 & PPP 000802 & E (1) & $0.54956(1)$ \\
\hline C3 & 0.5465 & 0.7 & PPP 001123 & $S(2)$ & $0.54747(2)$ \\
\hline $\mathrm{C} 4$ & 0.5324 & 0.9 & PPP 001795 & S (2) & $0.53345(2)$ \\
\hline C5 & 0.5312 & 1.1 & PPP 001787 & $\begin{array}{c}\text { Sc-Irr (1) } \\
\text { S0 (2) }\end{array}$ & $0.53156(1)$ \\
\hline \multirow[t]{2}{*}{ C6 } & 0.5305 & 1.1 & PPP 001802 & Sb-Sc (1) & $0.53094(1)$ \\
\hline & & & & $S(2)$ & 0.53007 (2) \\
\hline C7 & 0.5277 & 0.8 & PPP 001696 & S (2) & $0.52700(3)$ \\
\hline $\mathrm{C} 8$ & 0.5325 & 0.7 & PPP 001482 & $\begin{array}{c}\text { Sc-Irr (1) } \\
\text { S(2) }\end{array}$ & $\begin{array}{l}0.53257(1) \\
0.53215(2)\end{array}$ \\
\hline C9 & 0.5246 & 0.5 & [SED2002] 111 & $\begin{array}{c}\text { E/SO (4) } \\
\operatorname{Irr}(2)\end{array}$ & $0.52420(2)$ \\
\hline $\mathrm{C} 10$ & 0.5312 & 0.8 & - & - & - \\
\hline C11 & - & 0.1 & PPP 001264 & E (1) & $0.53070(1)$ \\
\hline F1 & 0.9009 & - & - & - & $0.90011(2)$ \\
\hline $\mathrm{F} 2$ & 0.5795 & - & PPP 001818 & $\mathrm{Sb}-\mathrm{Sc}(1)$ & $\begin{array}{l}0.57924(1) \\
0.57918(2)\end{array}$ \\
\hline F3 & 0.5667 & - & PPP 002130 & $\begin{array}{c}\text { Sc-Irr (1) } \\
\text { S (2) }\end{array}$ & $0.56686(1)$ \\
\hline F4 & 0.1867 & - & PPP 001358 & $\begin{array}{c}\text { Sc-Irr (1) } \\
\text { S (2) }\end{array}$ & $0.18727(1)$ \\
\hline F5 & 0.1573 & - & PPP 001403 & $\begin{array}{l}\operatorname{Irr}(4) \\
\text { S (2) }\end{array}$ & $0.15679(2)$ \\
\hline F6 & 0.0982 & - & PPP 001542 & S (2) & 0.09809 (2) \\
\hline F7 & - & - & - & Merger (2) & $0.91251(2)$ \\
\hline F8 & 0.4443 & - & PPP 001259 & Sc-Irr (1) & $0.44392(1)$ \\
\hline F9 & 0.3259 & - & PPP 000798 & - & $0.32561(2)$ \\
\hline F10 & 0.4947 & - & - & $\operatorname{Irr}(2)$ & 0.648 (2)(ph.) \\
\hline F11 & - & - & PPP 001478 & - & 0.319 (2)(ph.) \\
\hline
\end{tabular}

Column (1): object ID; Col. (2): redshift; Col. (3): the projected distance from the cluster center in Mpc; Col. (4): the name of the galaxy in Nasa Extragalactic Database (NED); Col. (5): the morphological type of the galaxy and its reference; Col. (6): redshift of the galaxy and its reference. Photometric redshifts are indicated with "(ph.)".

References: (1): (Ellingson et al. 1998); (2): (Moran 2008); (3): (Moran et al. 2007b); (4): (Stanford et al. 2002). All galaxy names given in Col. 4 begin with "MS 0451.6-0305:". [SED2002] $=$ Stanford+Eisenhardt+Dickinson. For C11, F7 and F11, we couldn't measure the redshifts using our data as explained in the text. The distance of $\mathrm{C} 11$ to the cluster center is calculated using its redshift from the literature.

the end, we had defined 20 slits for 22 targets, of which ten had spectroscopic information, six appropriate colors and six were just fillers. After data reduction, the sample turned out to have eleven cluster members. Five galaxies four of which are cluster members have no or very weak emission lines and are, therefore, not suitable for a kinematic analysis of their gas velocity fields.

To each galaxy we assign a number and put a "C" or an "F" in front of it, indicating whether it is a cluster member or a field galaxy. In Table 1, we give their redshifts and for the cluster members we give the projected distance from the center, together with some information taken from the literature (morphological type and redshift). For C11, F7 and F11 we could not determine the redshift from our data. F8 and C11 were observed within the same slit. They are so close together that their spectra can not be distinguished. The redshift that is calculated using the emission lines in the composite spectrum is $z=0.4443$. Ellingson et al. (1998) shows that C11 is an elliptical cluster member and that the emission we measure comes from F8. In the spectra of F7 


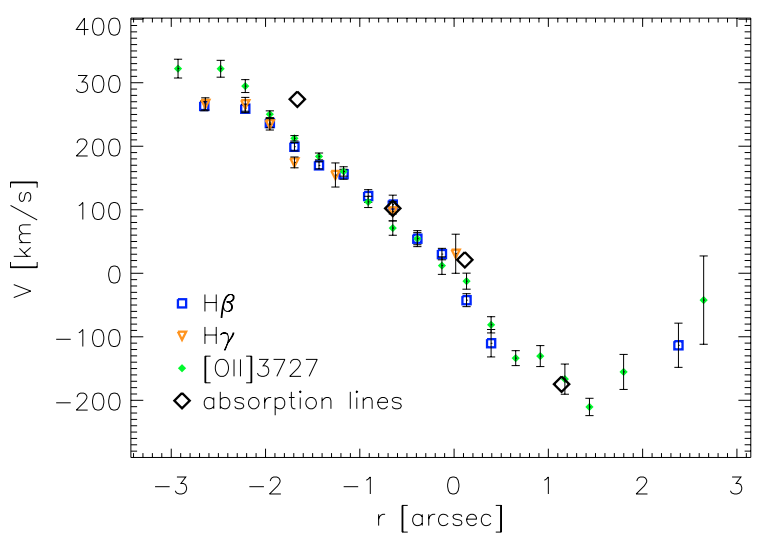

Fig. 3. The observed gas and stellar rotation curves of a cluster member (galaxy C8) extracted along the central slit with no correction for inclination and seeing. Both components have their principal motions along the same direction and with similar amplitude.

and F11, one strong emission line is visible and no other feature can be identified. In both cases, different possibilities for identification of the line rule out that these two galaxies are cluster members. Therefore they are flagged with an " $F$ " showing that they are field galaxies. In Table 1 we give a redshift of galaxy F7 and F11 from Moran (2008). The redshift of galaxy F11 is photometric. Since photometric redshifts have big uncertainties, we do not use it in our analysis. All cluster members are within $2 \mathrm{Mpc}$ from the center and most of them are closer than $1 \mathrm{Mpc}$, i.e. they are well inside one virial radius (or $R_{200}$ ) of the cluster.

Our spectra cover the wavelength range $5000 \AA \leq \lambda_{\text {obs }} \leq$ $8000 \AA$. Several lines can be observed within this interval depending on the redshift. In case of visible emission lines excited by different mechanisms we can use them to investigate the physical state of different gas clouds. We checked whether the kinematics derived from different lines are consistent with each other or whether there are prominent discrepancies that may indicate ongoing or recent interaction processes. If strong absorption lines are present, we also compare the stellar rotation with the gas kinematics (see Sect. 2.5 and, e.g. Fig. 3).

In case of cluster spirals, the visible emission lines are [OII]3727, $\mathrm{H} \gamma, \mathrm{H} \beta,[\mathrm{OIII}] 4959$ and [OIII]5007, of which [OII] 3727 is usually the strongest. The rotation curves extracted along the central slit using prominent emission lines of a cluster member $(\mathrm{C} 8)$ are shown together with its stellar rotation curve in Fig. 3. The redshift range of field galaxies is $0.1 \leq z \leq 0.9$. For the highest redshift in this range, the only visible line in the spectrum is [OII] 3727 doublet while for small redshifts several lines are visible. For example the emission lines covered in the spectrum of $\mathrm{F} 5$ at $z=0.1573$ are $\mathrm{H} \gamma, \mathrm{H} \beta$, [OIII]4959, [OIII]5007, OI, $\mathrm{H} \alpha$, [NII]6583, [SII]6716 and [SII]6730. There are three foreground galaxies for which the $\mathrm{H} \alpha$ line is visible (F4, F5 and F6) and it is the strongest line in all cases. In Fig. 4 we show the rotation curves extracted along the central slit using the prominent lines of F5.

In the Appendix, we give some information about each galaxy. In case the galaxy has emission, we present:

$a-$ the HST-ACS image of the galaxy in the $I$ band;

$b$ - rotation curves of different emission lines (and for some cases based on the absorption lines) extracted along the central slit without correction for inclination and seeing;

$c$ - position angles of kinematic and photometric axes as a function of radius;

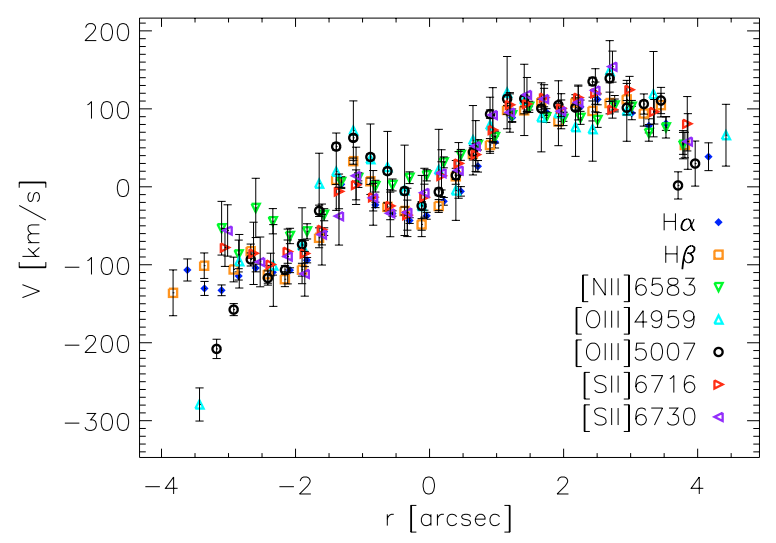

Fig. 4. Rotation curves of a foreground galaxy at $z=0.1573$ (galaxy F5) extracted along the central slit using the prominent emission lines in its spectrum (no correction for inclination and seeing is applied).

$d-$ rotation curves extracted along the central slit and the kinematic major axis;

$e$ - velocity field obtained using the strongest line in the spectrum;

$f-$ velocity map reconstructed using 6 harmonic terms;

$g$ - residual of the velocity map and the reconstructed map;

$h$ - normalized flux map of the line used for constructing the velocity field;

$i-$ simple rotation map constructed for position angle and ellipticity fixed to their global values;

$j-$ residual of the velocity map and the simple rotation model;

$k$ - position angle and flattening as a function of radius;

$l-k_{3} / k_{1}$ and $k_{5} / k_{1}$ (from the analysis where position angle and ellipticity are fixed to their global values) as a function of radius.

There are some emission-line galaxies for which we did not analyze the velocity fields, and therefore no figures are given related to their velocity fields: galaxies F1, F8, C5 and C6. Galaxy F8 lies very close to galaxy $\mathrm{C} 11$, and their FORS spectra could not be separated. Galaxy F1 was observed with only 1 slit position. Galaxies C5 and C6 are interacting, and have extremely irregular velocity fields.

\subsection{Kinematic analysis methods}

The redshifts were calculated using all visible emission lines and a few prominent absorption lines in the integrated 1D-spectra of the central slit. Then, for each galaxy and for each emission line, the photometric center of the galaxy was determined by fitting a Gaussian to the continuum light distribution in the spatial direction averaged over $160 \AA$ excluding the emission line itself. For each emission line separately, line centers and widths were measured along the spatial axis by Gaussian fits row by row, after the underlying continuum had been subtracted. The measurements were done by starting at the photometric center and continuing outwards on both sides. In regions of low signal, rows were first binned until a minimum flux in the emission line was reached. This means that in the outer regions of a galaxy, not every pixel of the grid shown in Fig. 2 corresponds to an independent data point for the velocity field (Fig. 5, top left). Finally, rotation curves were constructed. This was done for the spectra at all three positions (Fig. 5, bottom row).

To construct the $3 \mathrm{D}$-velocity fields from the three rotation curves, all measurements were transformed to a common 

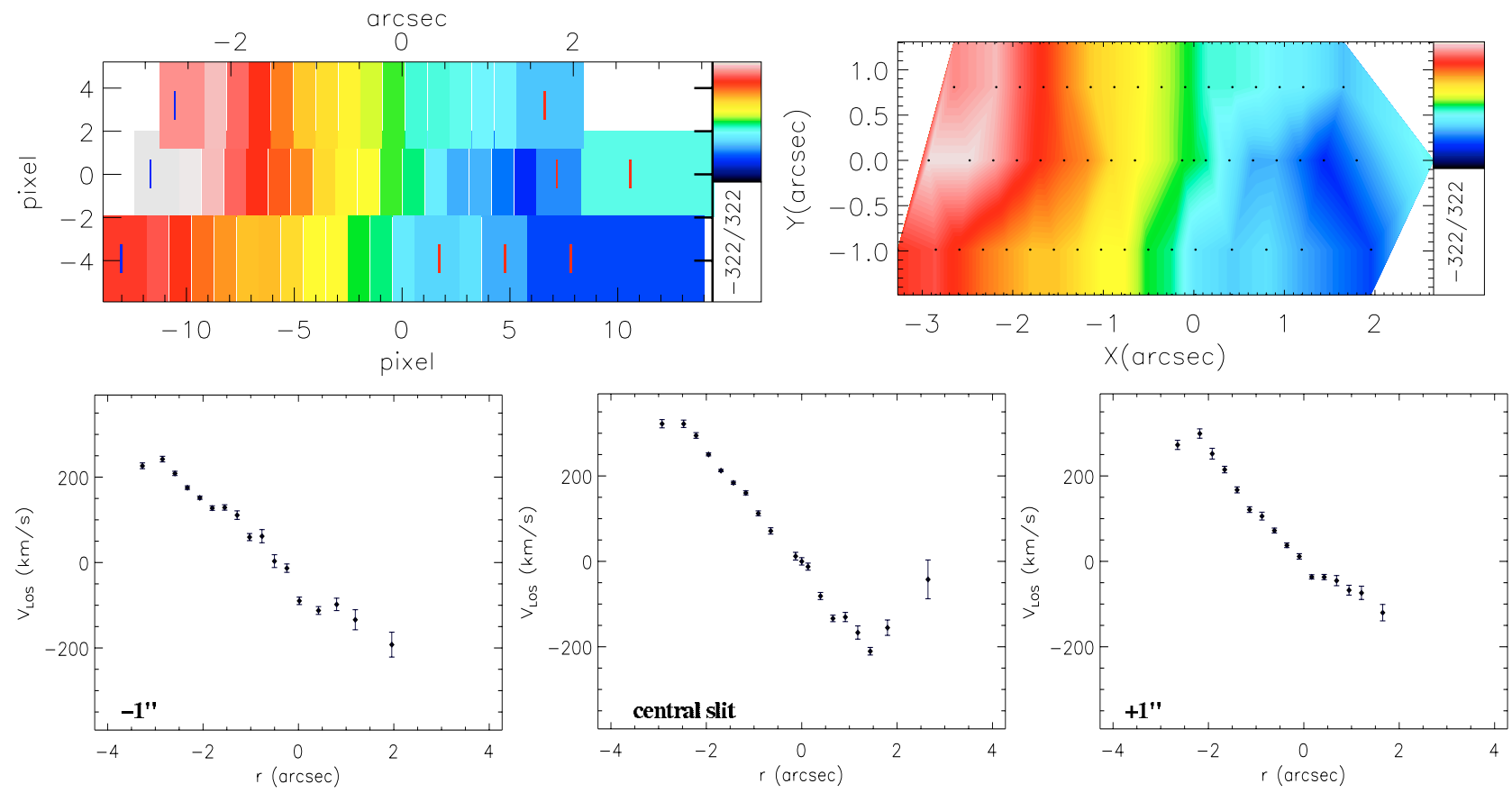

Fig. 5. Top left: pixel map of the galaxy in Fig. 2 (galaxy C8), based on [O II] 3727 emission line measurements. In case that the pixels are binned, the flux weighted position is indicated with a red/blue line on the map. The intersection between the pixel rows is caused by the spatial offsets between the masks mentioned in Sect. 2.3. Velocities are given in $\mathrm{km} \mathrm{s}^{-1}$. Top right: the velocity map visualizing the data on the left. Velocities are interpolated linearly. Bottom left: rotation curve extracted along the slit $1^{\prime \prime}$ below the central one. Bottom middle: rotation curve extracted along the central slit. Bottom right: rotation curve extracted along the slit 1" above the central one.

coordinate system defined by (the continuum center of) the central slit. The spatial offsets of the three masks relative to each other were determined using spectra of stars observed with 2 slits oriented perpendicular to each other so that the shift along both $x$ and $y$ axes could be determined. Because of the shift, the slit offsets along the minor axis of the galaxies between the three setups were not exactly $\pm 1^{\prime \prime}$ as intended, leading to a slightly different coverage of each galaxy from the ideal version of the grid shown in Fig. 2. The maximum absolute difference that we measured between the position of each mask is $\sim 1.5$ pixels. Taking this into account, the observed velocity map was constructed using linear interpolation (Fig. 5, top right). We also made emission line strength maps (see the Appendix). Here we will explain how we analyzed the velocity maps, and we derived parameters such as kinematic position angle $(\Gamma)$ and kinematic inclination $(i)$ (Fathi et al. 2005).

To analyze the velocity fields, we used the kinemetry package of Krajnović et al. (2006) that was extensively tested and used for SAURON IFU observations of local galaxies. For a rotating disk the line of sight velocity is given by:

$V_{\mathrm{los}}(R)=V_{\mathrm{sys}}+V_{\mathrm{rot}}(R) \cos \psi \sin i$

where $V_{\text {los }}$ is the observed line of sight velocity, $V_{\text {sys }}$ is the systemic velocity, $V_{\text {rot }}$ is the circular velocity and $(R, \psi)$ are polar coordinates in the plane of the galaxy with $\psi$ measured from the apparent major axis. According to Eq. (1), the observed velocity along a certain orbit can be expressed as a simple cosine form when plotted versus azimuthal angle. In general, since the motion of gas in galaxies can often be explained by rotation plus a smaller perturbation, one can generalise Eq. (1) to

$V(R, \psi)=A_{0}(R)+\sum_{n=1}^{N} A_{n}(R) \sin (n \psi)+B_{n}(R) \cos (n \psi)$.
A grid of position angle - flattening combinations covering the whole parameter space is constructed for each radius, where flattening is defined as $q=b / a=1$-ellipticity. Fourier analysis is performed on the velocity profiles extracted along each ellipse. The best sampling ellipse is determined by minimizing (see Krajnović et al. 2006):

$\chi^{2}=A_{1}^{2}+A_{3}^{2}+B_{3}^{2}$.

For an infinitesimally thin disk the flattening of the isovelocity contours is related to the inclination by $q=b / a=\cos i$. For every galaxy we determined a global position angle and inclination by taking the median of the values at several radii outside half the FWHM (full width at half maximum) of the seeing. In Fig. 7 we show, for a field galaxy in our sample, the comparison between the rotation curves extracted along the central slit and along the kinematic major axis. This reveals a very important advantage of measuring the full velocity field instead of just one rotation curve. The rotation curve along the kinematic major axis clearly reaches a higher value of $V_{\max }$ than the photometric major axis rotation curve, which is mostly used for the Tully-Fisher analysis.

To determine the deviations from simple rotation, we repeated the Fourier analysis of the velocity maps fixing the position angle and the inclination of the orbits to these global values. By subtracting the model of a regular rotation disk $B_{1} \cos \psi$ from the observed velocity field, non-circular motions and perturbations can be seen in the residual image (Fig. 6). We can quantify them using the Fourier terms $A_{n}$ and $B_{n}$. We use the amplitude of the $n$th order Fourier term, defined in Krajnović et al. (2006):

$k_{n}=\sqrt{A_{n}^{2}+B_{n}^{2}}$.

The coefficients $k_{3}$ and $k_{5}$ (Eq. (4)) represent deviations from simple rotation. We do not fit the even Fourier terms, since they 

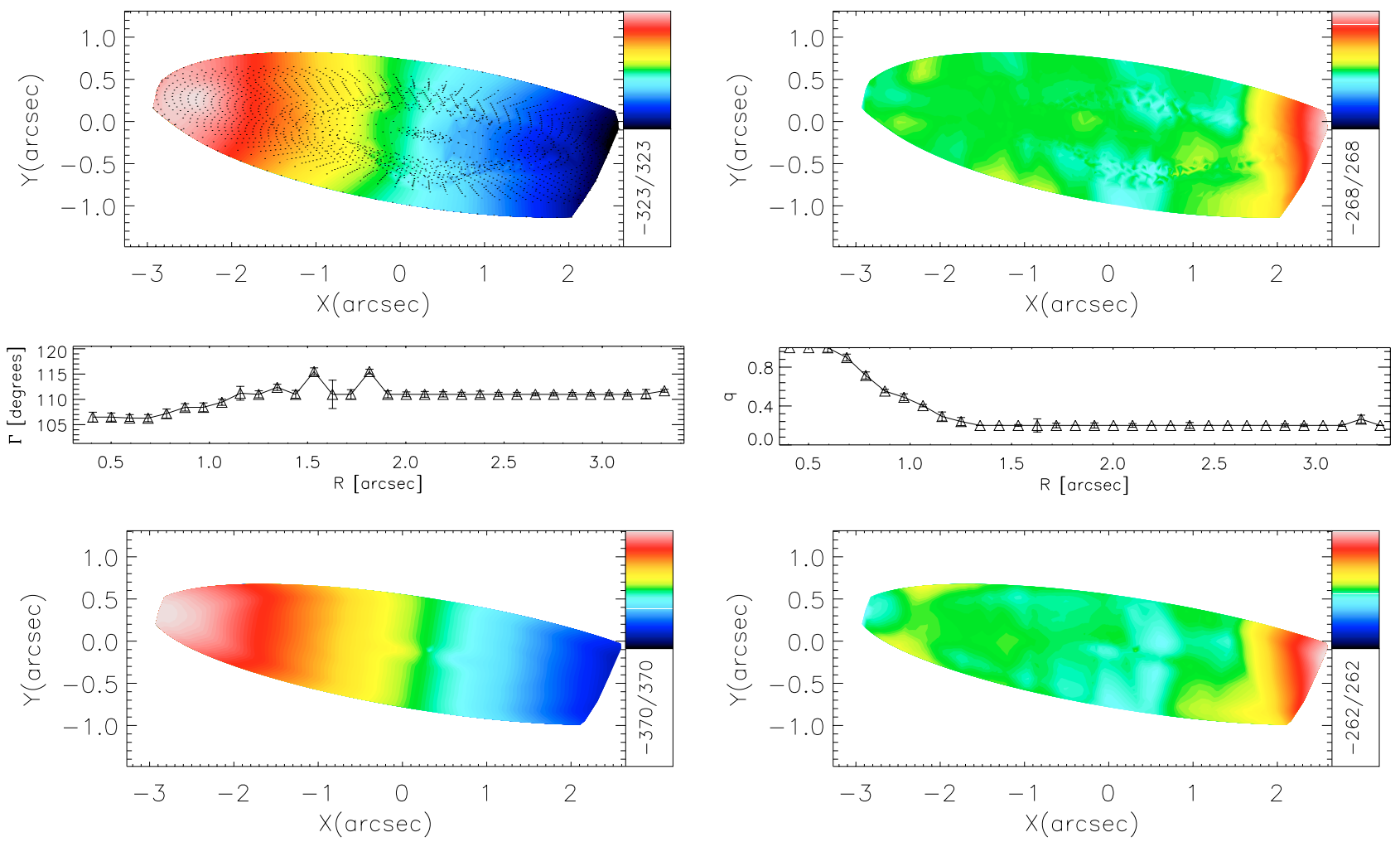

Fig. 6. Top left: reconstructed velocity field of the galaxy in Fig. 2 (galaxy C8) constructed using 6 harmonic terms of the Fourier analysis performed by kinemetry. Top right: residual of the observed velocity map and the reconstructed map given on the left. Middle: kinematic position angle $\Gamma$ and flattening $q$ as a function of distance to the kinematic center. Bottom left: velocity field of the best fitting rotating disk $\left(B_{1} \cos \psi\right) \operatorname{constructed~fixing}$ the position angle $\Gamma$ and flattening $q$ to their global values. Bottom right: residual of the observed velocity map and the rotating disk velocity field given on the left.

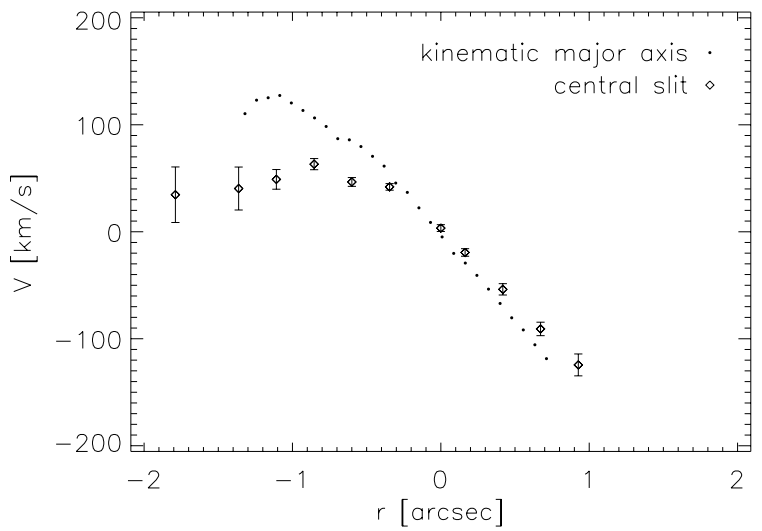

Fig. 7. Rotation curves of a field galaxy at $z=0.5667$ (galaxy F3) extracted along the central slit which is $3^{\circ}$ misaligned w.r.t. the photometric major axis, and the kinematic major axis.

should vanish if the velocity fields are point-anti-symmetric. To measure the total deviation, we also define an additional coefficient:

$k_{3,5}=\sqrt{A_{3}^{2}+B_{3}^{2}+A_{5}^{2}+B_{5}^{2}}$.

Before running kinemetry the kinematic center has to be known. To determine it we use the fact that when the velocity field is regular the velocity gradient is the highest at the kinematic center (Arribas et al. 1997). The peak of the squared sum of the velocity gradient in perpendicular directions is taken to be the kinematic center. In case that there are shocks in the gas, this method possibly does not work, but then the kinematic center can generally be found as a secondary peak in the velocity gradient map. For instance for galaxy F6 (Appendix, Fig. A13), a galaxy that has a kinematically decoupled core (KDC), we took the peak of the gradient in the KDC as the kinematic center although the gradient in the transition region was much higher.

\subsection{Stellar kinematics}

In addition to the analysis of the gas velocity fields, we can also investigate the kinematics of the stellar component, if absorption lines are present in the galaxy spectra with sufficient $S / N$. This prerequisite is mostly fulfilled only for the central slit spectrum, where the surface brightness is large enough. In these cases, we determine the stellar rotation and velocity dispersion from several strong absorption lines using the pPXF software (Cappellari \& Emsellem 2004). This program fits different linear combinations of stellar templates to a wavelength region in pixel space. As templates, we use SSP (Single age and metallicity Stellar Population) models created with the MILES library (Sánchez-Blázquez et al. 2006). In Fig. 3, we compare, as an example, the gas and stellar rotation curves of a cluster member, both extracted along the central slit.

In our sample, there are 6 galaxies for which we could extract the stellar rotation curve. For those, we have compared the rotation of stars and gas (see Appendix, Figs. B.1b, B.3b-B.6b, B.9b). In the case of galaxy C8 and F2 they show a similar behaviour. For galaxy C5 and galaxy C9, there is a discrepancy between the two curves. Galaxy $\mathrm{C} 5$ is interacting with its companion (Fig. B.3). Galaxy C9 has a big misalignment between 
its kinematic and photometric axes. It appears that the gas component in both galaxies is disturbed. In case of galaxy C7, which has an irregular gas velocity field, while the $\mathrm{H} \beta$ and $\mathrm{H} \gamma$ rotation curves do not show a big discrepancy with the stellar one, the $[\mathrm{O}$ II $] 3727$ curve does show a somewhat larger discrepancy. Galaxy $\mathrm{C} 1$ has very weak [O II]3727 emission, so a comparison in this case would not give reliable information.

In all cases, the stellar rotation curve is regular. The cross sections for gas clouds in spiral disks are large enough that disturbances in the gas velocities are triggered rather easily, whereas stellar orbits are collisionless (Sarzi et al. 2006). Therefore, gas velocity fields are expected to be more distorted and richly structured than their stellar counterparts (e.g. Dumas et al. 2007). However, comparing with local spiral galaxies (see Falcón-Barroso et al. 2006) one sees that in the inner regions of galaxies such discrepancies between stars and gas are rare in the local Universe. For the local cluster members, the situation might be different. From $\mathrm{H} \alpha$ morphologies of galaxies in the Virgo cluster Koopmann \& Kenney (2004) find that about half of their Virgo spiral galaxies have truncated $\mathrm{H} \alpha$ disks, while several spiral galaxies have asymmetric $\mathrm{H} \alpha$ enhancements at the outer edge of their disks, and at least two highly inclined spiral galaxies have extraplanar concentrations of HII regions. Chemin et al. (2006) investigated $\mathrm{H} \alpha$ velocity fields of local cluster spirals, and showed that they exhibit typical kinematical perturbations like streaming motions along spiral arms, twist of the major axis, $Z$ shape of velocities due to the presence of a bar, decoupled nuclear spiral or misalignment between photometric and kinematic major axes. In general, however, these perturbations are minor: for most galaxies the kinematics of the gas can be considered a perturbation to the stellar kinematics.

\section{Photometry}

\subsection{Groundbased photometry}

To study the photometry of our galaxies we used data from the HST and ground-based telescopes. To determine luminosities and colours of our target galaxies we make use of photometry obtained at ground-based telescopes. For the cluster MS 0451, we have taken direct images with FORS2 at the VLT (ESO PID 66.A-0547). Frames in the $V, R_{\mathrm{C}}$ and $I_{\mathrm{C}}$ filters were reduced in the standard manner (bias and flatfield correction, registering, astrometric calibration using IRAF $^{1}$ tasks) and combined to yield total exposure times of 3300, 1800 and $2400 \mathrm{~s}$, respectively. Photometric calibration was achieved via standard stars from Landolt (1992). For determination of colours, the final combined $V, R$ and $I$ images were convolved with a 2 D-Gaussian filter to a common seeing of 0 . $^{\prime} 9$. Photometry was performed using the SExtractor package (Bertin \& Arnouts 1996). While MAG_BEST magnitudes are taken as total magnitudes, apertures with diameter 2.'0 were used to compute colours. All magnitudes were corrected for Galactic extinction by $A_{V}=0.110$, $A_{R}=0.088$ and $A_{I}=0.064$ given for the cluster coordinates in NED (NASA/IPAC Extragalactic Database), which are based on $E(B-V)=0.033$ (Schlegel et al. 1998). The three filter magnitudes were transformed to rest-frame Johnson- $B$ using the $k$-correction algorithm by Blanton \& Roweis (2007). The absolute total luminosities of the galaxies are the second parameter entering the Tully-Fisher relation. All derived magnitudes of the

\footnotetext{
${ }_{1}$ IRAF is distributed by the National Optical Astronomy Observatories, which is operated by the Association of Universities for Research in Astronomy, Inc., under cooperative agreement with the National Science Foundation.
}

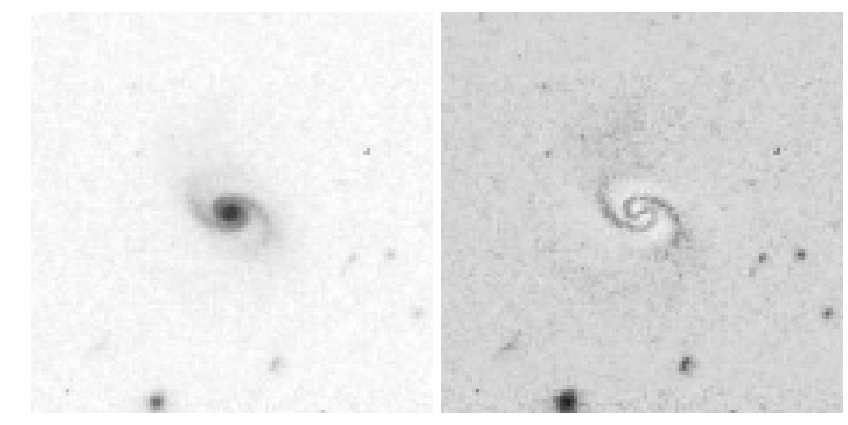

Fig. 8. Left: the HST-ACS I band image of a cluster member (Galaxy C2). Right: the residual of the exponential disk + Sérsic bulge fit.

objects for which we have spectra are listed in Table C.1. The tabulated $M_{B}$ magnitudes of the spiral galaxies are not corrected for internal dust (inclination).

\subsection{HST imaging and structural analysis}

For the cluster MS 0451 presented here, we exploit existing imaging in the ACS $/ F 814 W$ filter from the ST-ECF archive. We basically used the pipeline reduced images but with an additional cosmic ray rejection routine.

Structural parameters of the galaxy disks such as scale length, inclination, ellipticity and position angle were determined by decomposing the $3 \mathrm{D}$-surface brightness distribution of the target objects into an exponential disk and a Sérsic bulge using the GALFIT algorithm (Peng et al. 2002) (Table C.2).

The model-subtracted residual images reveal additional structural features like spiral arms (Fig. 8) and bars, or signatures of ongoing or recent interaction processes such as tidal tails that can be compared to the kinematic tracers.

To further investigate the structure of these galaxies we also determined the asymmetry and concentration index of the galaxies (e.g. Abraham et al. 1994). To calculate the concentration parameter, we use the ellipticity, position angle and central coordinates (derived with SExtractor on the second order moments of the images) to define an elliptical area that includes all the flux above $2 \sigma$ of the sky level and an elliptical area for which the semi-major axis is $30 \%$ of the outer one (Eq. (6)).

$C=\frac{\sum \sum_{i, j \in E(0.3)} I_{i j}}{\sum \sum_{i, j \in E(1)} I_{i j}}$

For the asymmetry index, we applied the definition and centering method of Conselice et al. (2000). The sky subtracted galaxy image and its counterpart rotated by 180 degrees are compared with each other in an elliptical area including all flux above the $1.5 \sigma$ sky level. A $3 \times 3$ grid was defined around the initial center to search for the coordinates that give the smallest asymmetry. The distance from the central point to the eight surrounding points was set to $0 . ' 1$. The grid was reconstructed around the coordinates that gave the smallest asymmetry in the previous grid. That was done until the smallest value was measured at the center. Similarly, the asymmetry of a blank region was calculated in the sky subtracted image using the same elliptical mask that was used for the galaxy. To correct for the contribution of the noise, this "blank asymmetry" was then subtracted from the asymmetry of the galaxy:

$A=\min \left(\frac{\sum\left|I_{0}-I_{180}\right|}{\sum\left|I_{0}\right|}\right)-\left(\frac{\sum\left|B_{0}-B_{180}\right|}{\sum\left|I_{0}\right|}\right)$ 


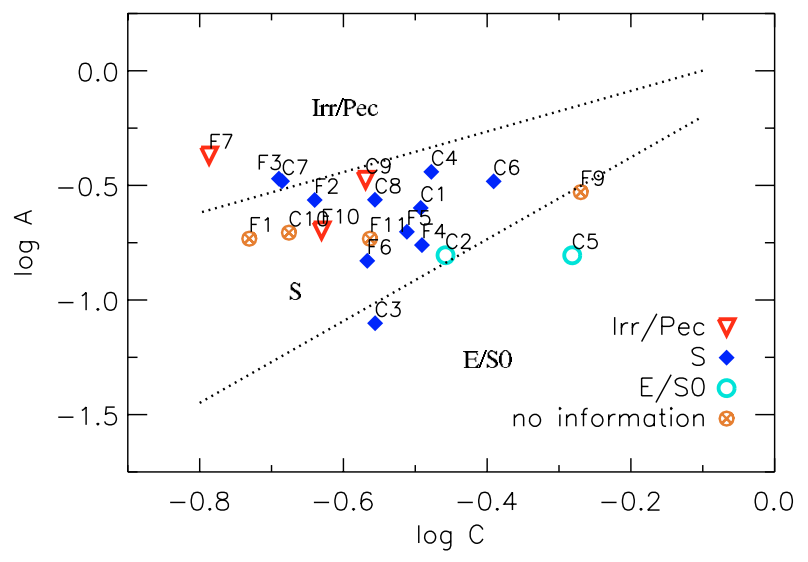

Fig. 9. Distribution of our galaxies in the $A-C$ plane. Symbols are used to indicate morphologies of these galaxies. The references for this information are given in Table 1 . In case inconsistent morphology information was given in different references, we used the morphologies from Moran (2008). The dashed lines are the selection limits taken from Menanteau et al. (2006).

where $I$ represents the image pixel values and $B$ represents the blank region pixel values. The asymmetry and concentration parameters of our galaxies are given in Table C.1 in the Appendix. This information can be used for morphological classification. We present our galaxies on the $A-C$ plane together with their morphologies from the literature (Table 1) and the selection limits from Menanteau et al. (2006) in Fig. 9.

\section{Analysis}

Here we want to classify our galaxies using a number of parameters that are expected to be good indicators of the irregularity of velocity fields: $k_{3,5} / k_{1}, \sigma_{\mathrm{PA}}$ and $\Delta \phi$. The deviation from simple rotation was measured using the $k_{3,5} / k_{1}$ parameter, described in Sect. 2.4. It is the squared sum of the higher order Fourier terms (normalized by the rotation velocity) obtained for a fit with a fixed position angle and ellipticity. For each galaxy we obtained an average $k_{3,5} / k_{1}$ value. $\sigma_{\mathrm{PA}}$ is the standard deviation of the kinematic position angle within a galaxy and $\Delta \phi$ is the absolute value of the average difference between the kinematic and photometric position angle at several radii. The radial extent over which these parameters have been calculated depends on the distance of the galaxy, its surface brightness and its ionized gas contents. Since not at every radius enough gas is present we could not fit the velocity fields at all radii for all galaxies. The maximum radius for which we could measure these parameters for each galaxy $\left(R_{\max }\right)$ is given in Table 2. $\sigma_{\mathrm{PA}}, \Delta \phi$ and $k_{3,5} / k_{1}$ are calculated between $r=0.39$ and the $R_{\max }$ (Table 2). The errors in these parameters are the variance of the parameters in the range of the observations.

In Fig. 10, we show how the galaxies are distributed in the parameter space of $k_{3,5} / k_{1}, \Delta \phi$ and $\sigma_{\text {PAA }}$. To be able to decide whether these velocity fields are regular we should determine a regularity treshold for each parameter. To do that, we measured the parameters for the $\mathrm{H} \alpha$ velocity fields of the SINGS local sample (Daigle et al. 2006) in which most galaxies have regular kinematics. Among the velocity fields we were provided with (all but NGC 925, NGC 2403, UGC 5423, NGC 3198, NGC 4321, NGC 6946) we excluded from the analysis the galaxies that have magnitudes which are very different from the ones in our sample (m81dwb, NGC 2915) and the velocity fields that are very noisy (IC 4710 and NGC 5398). For every galaxy we ran
Table 2. Parameters quantifying the irregularity of the gas kinematics measured for our sample.

\begin{tabular}{ccccc}
\hline \hline ID & $R_{\max }$ & $\sigma_{\mathrm{PA}}$ & $\Delta \phi$ & $k_{3,5} / k_{1}$ \\
$(1)$ & $(2)$ & $(3)$ & $(4)$ & $(5)$ \\
\hline C7 & 13.8 & 23 & $68 \pm 26$ & $0.32 \pm 0.20$ \\
C8 & 20.9 & 2 & $9 \pm 6$ & $0.06 \pm 0.05$ \\
C9 & 9.2 & 19 & $66 \pm 20$ & $0.10 \pm 0.03$ \\
C10 & 11.7 & 9 & $14 \pm 9$ & $0.26 \pm 0.08$ \\
F2 & 10.5 & 2 & $35 \pm 37$ & $0.08 \pm 0.02$ \\
F3 & 10.0 & 7 & $39 \pm 9$ & $0.07 \pm 0.05$ \\
F4 & 5.5 & 21 & $18 \pm 20$ & $0.30 \pm 0.19$ \\
F5 & 11.3 & 5 & $46 \pm 9$ & $0.08 \pm 0.03$ \\
F6 & 4.4 & 29 & $57 \pm 44$ & $0.27 \pm 0.18$ \\
F7 & 14.1 & 3 & $0 \pm 11$ & $0.05 \pm 0.02$ \\
F10 & 8.4 & 8 & $1 \pm 7$ & $0.25 \pm 0.05$ \\
F11 & 1.7 & 5 & $18 \pm 5$ & $0.05 \pm 0.02$ \\
\hline
\end{tabular}

Column (1): object ID; Col. (2): maximum radius for which kinematic parameters could be calculated. The conversion from arcsecond into kpc was done as explained in Wright (2006); Col. (3): standard deviation of the kinematic position angle $\left(\sigma_{\mathrm{PA}}\right)$; Col. (4): mean misalignment between the kinematic and photometric position angles $(\Delta \phi)$; Col. (5): mean $k_{3,5} / k_{1}$ of the analysis done while fixing the position angle and the ellipticity to their global values.

For galaxy F11, only the photometric redshift is available. Since photometric redshifts have big uncertainties, we give $R_{\max }$ of this galaxy in arcseconds. $k_{3,5} / k_{1}$ of galaxy F5, $\Delta \phi$ of the galaxies that have $\epsilon \leq 0.1$ (galaxy F2) and all parameters for galaxy F10 are rather meaningless as explained in Sect. 4.1. Therefore they are excluded from the analysis.

kinemetry to determine the parameters $k_{3,5} / k_{1}, \Delta \phi$ and $\sigma_{\text {PA }}$. To be able to calculate $\Delta \phi$, the photometric position angle should be known as a function of radius. To measure that, we used the red Palomar DSS images (6450 ̊), apart from NGC 3521, NGC 3621 and NGC 5713, for which we used blue UK-Schmidt images (4680 ̊), also from the DSS. All parameters are given in Table 3. It is pointed out in Daigle et al. (2006), Table 1 that some of these galaxies are peculiar. These galaxies are indicated next to their ID in Table 3. We plotted the parameters against each other for both the local sample and our sample together in Fig. 11. Using the location of the local galaxies that are not peculiar and do not belong to the Virgo cluster, we defined a limit for each parameter below which we call the gas kinematics "regular". These regularity criteria are: $\Delta \phi \leq 25, \sigma_{\mathrm{PA}} \leq 20$ and $k_{3,5} / k_{1} \leq 0.15$.

To check whether the 3 parameters $k_{3,5} / k_{1}, \Delta \phi$ and $\sigma_{\mathrm{PA}}$ are indicators of the same phenomena, and correlate with each other, we calculated the uncertainty weighted linear Pearson correlations separately for the two samples. The correlation coefficients for each relation are given in the first two rows of Table 4. $\sigma_{\mathrm{PA}}$ and $k_{3,5} / k_{1}$ seem to correlate with each other while the correlation between $\Delta \phi$ and $\sigma_{\mathrm{PA}}$ holds only for the higher redshift sample and the correlation between $\Delta \phi$ and $k_{3,5} / k_{1}$ holds only for the local sample. We will discuss this result in more detail in Sect. 5.

We compared the distributions of $k_{3,5} / k_{1}, \Delta \phi$ and $\sigma_{\mathrm{PA}}$ for the local and higher redshift galaxies (Fig. 12). While some galaxies in our sample have $k_{3,5} / k_{1}$ values that are far above the region where the local galaxies are distributed, this is not the case for the $\sigma_{\mathrm{PA}}$ and $\Delta \phi$ parameters. Galaxies in both samples are distributed within similar ranges. $\sigma_{\mathrm{PA}}$ of galaxy $\mathrm{F} 6$ in our sample is $9^{\circ}$ above the highest value among the local galaxies. This galaxy has a kinematically decoupled core and that causes the $\sigma_{\mathrm{PA}}$ to be large. All local galaxies that have a large $\Delta \phi$ value are either peculiar or a member of the Virgo Cluster. 

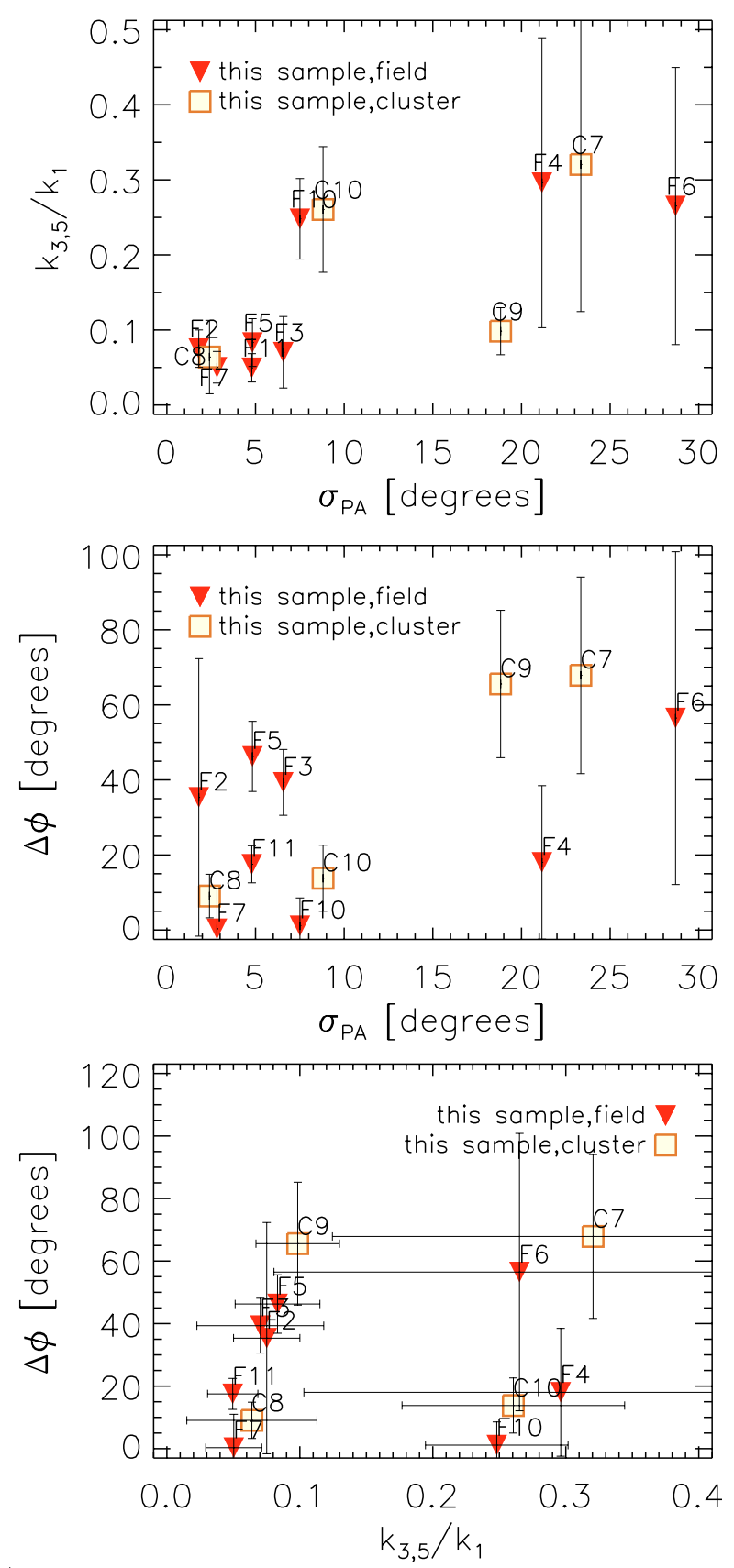

Fig. 10. Top: standard deviation of the kinematic position angle $\left(\sigma_{\mathrm{PA}}\right)$ versus mean $k_{3,5} / k_{1}$. Middle: mean misalignment between kinematic and photometric axes $(\Delta \phi)$ versus standard deviation of kinematic position angle $\left(\sigma_{\mathrm{PA}}\right)$. Bottom: mean $k_{3,5} / k_{1}$ versus mean misalignment between kinematic and photometric axes $(\Delta \phi)$.

Using the distributions for the cluster and field galaxies in our sample (Fig. 12), we investigate the effect of the environment on the gas kinematics. All 4 cluster galaxies are distributed in a similar range as the field galaxies. The fraction of galaxies that have irregular gas kinematics in the whole sample, among the field and cluster galaxies separately, is given in Table 5. Without distinguishing between different criteria we find that $75 \pm 22 \%$ of the cluster members, $50 \pm 18 \%$ of the field galaxies and $58 \pm$ $14 \%$ of the whole sample have irregular gas kinematics. These numbers are underestimates because the spatial resolution of the $z \approx 0.5$ observations is low. In Appendix A we show that the
Table 3. Parameters quantifying the irregularity of the gas kinematics measured for the local sample.

\begin{tabular}{cccc}
\hline \hline ID & $\sigma_{\mathrm{PA}}$ & $\Delta \phi$ & $k_{3,5} / k_{1}$ \\
$(1)$ & $(2)$ & $(3)$ & $(4)$ \\
\hline NGC 0628 & 18 & $9 \pm 35$ & $0.12 \pm 0.04$ \\
NGC 2976 (pec) & 6 & $3 \pm 17$ & $0.08 \pm 0.03$ \\
NGC 3031 & 19 & $2 \pm 19$ & $0.14 \pm 0.08$ \\
NGC 3049 & 20 & $18 \pm 20$ & $0.11 \pm 0.07$ \\
NGC 3184 & 7 & $21 \pm 57$ & $0.12 \pm 0.06$ \\
NGC 3521 & 2 & $3 \pm 3$ & $0.05 \pm 0.03$ \\
NGC 3621 & 2 & $5 \pm 20$ & $0.05 \pm 0.02$ \\
NGC 3938 & 4 & $11 \pm 25$ & $0.09 \pm 0.04$ \\
NGC 4236 & 5 & $11 \pm 5$ & $0.07 \pm 0.02$ \\
NGC 4536 & 5 & $4 \pm 13$ & $0.07 \pm 0.03$ \\
NGC 4569(Virgo) & 10 & $19 \pm 11$ & $0.06 \pm 0.02$ \\
NGC 4579(Virgo) & 10 & $36 \pm 16$ & $0.17 \pm 0.10$ \\
NGC 4625 (pec) & 6 & $57 \pm 52$ & $0.12 \pm 0.06$ \\
NGC 4725 (pec) & 12 & $30 \pm 28$ & $0.11 \pm 0.06$ \\
NGC 5055 & 3 & $4 \pm 4$ & $0.03 \pm 0.02$ \\
NGC 5194 (pec) & 5 & $49 \pm 43$ & $0.05 \pm 0.02$ \\
NGC 5713(pec) & 8 & $54 \pm 54$ & $0.06 \pm 0.02$ \\
NGC 7331 & 6 & $9 \pm 11$ & $0.10 \pm 0.04$ \\
\hline
\end{tabular}

Column (1): object ID; Col. (2): standard deviation of the kinematic position angle $\left(\sigma_{\mathrm{PA}}\right)$; Col. (3): mean misalignment between the kinematic and photometric position angles $(\Delta \phi)$; Col. (4): mean $k_{3,5} / k_{1}$ of the analysis done while fixing the position angle and the ellipticity to their global values.

Peculiar galaxies and members of Virgo Cluster are indicated on the first column with "(pec)/(Virgo)" next to their name. $\Delta \phi$ of the galaxies that have $\epsilon \leq 0.1$ (NGC 628, NGC 3184, NGC 3938, NGC 5713) have large errors as explained in Sect. 4.1, and are therefore excluded from the analysis.

fractions given above are lower limits to the real number of irregular galaxies.

We now checked whether there is a correlation between the irregularity in the gas kinematics and some other parameters derived from the photometry: the absolute $B$ magnitude, disk scale length, asymmetry and concentration index (see Sect. 3.2). We did this for our sample and the local sample separately. For the local sample, we do not have all these parameters, so we give the correlations only for the absolute magnitude in the $B$ band $\left(M_{B}\right)$ and the disk scale length. We calculated their disk scale length assuming $R_{\mathrm{d}} \sim 0.25 R_{25}$ ( $R_{25}$ values are taken from Daigle et al. 2006). We find a correlation between $M_{B}$ and $k_{3,5} / k_{1}$ only for our sample (Fig. 13). The distribution of the local sample galaxies in the plot is showing that galaxies with very different $M_{B}$ values can have very similar $k_{3,5} / k_{1}$ parameters. This correlation is therefore most probably spurious.

We do not find a correlation between the irregularity in the gas kinematics and either the concentration index $\mathrm{C}$, which is an indicator of the morphological type of the galaxy, or the photometric asymmetry (Table 4).

We have investigated whether the irregularity in the gas kinematics of the galaxy correlates with its ionized gas contents. This is calculated by measuring the integrated emission line flux and correcting it for the distance of the galaxy (multiplying it by $d^{2}$ ). Since the redshift interval of the sample is large $(0.1 \leq z \leq 0.9)$ we do not have the same lines for every galaxy. The strongest emission line that we have for all cluster galaxies is [OII $] 3727$. Using the mean emission line flux ratios derived from our spectra $(\mathrm{H} \alpha /[\mathrm{OII}] 3727=6.60, \mathrm{H} \beta /[\mathrm{OII}] 3727=0.82$, $[\mathrm{OIII}] 5007 /[\mathrm{OII}] 3727=1.77)$, we calibrated the flux from other lines to the [OII]3727 flux. The emission line that was used for 

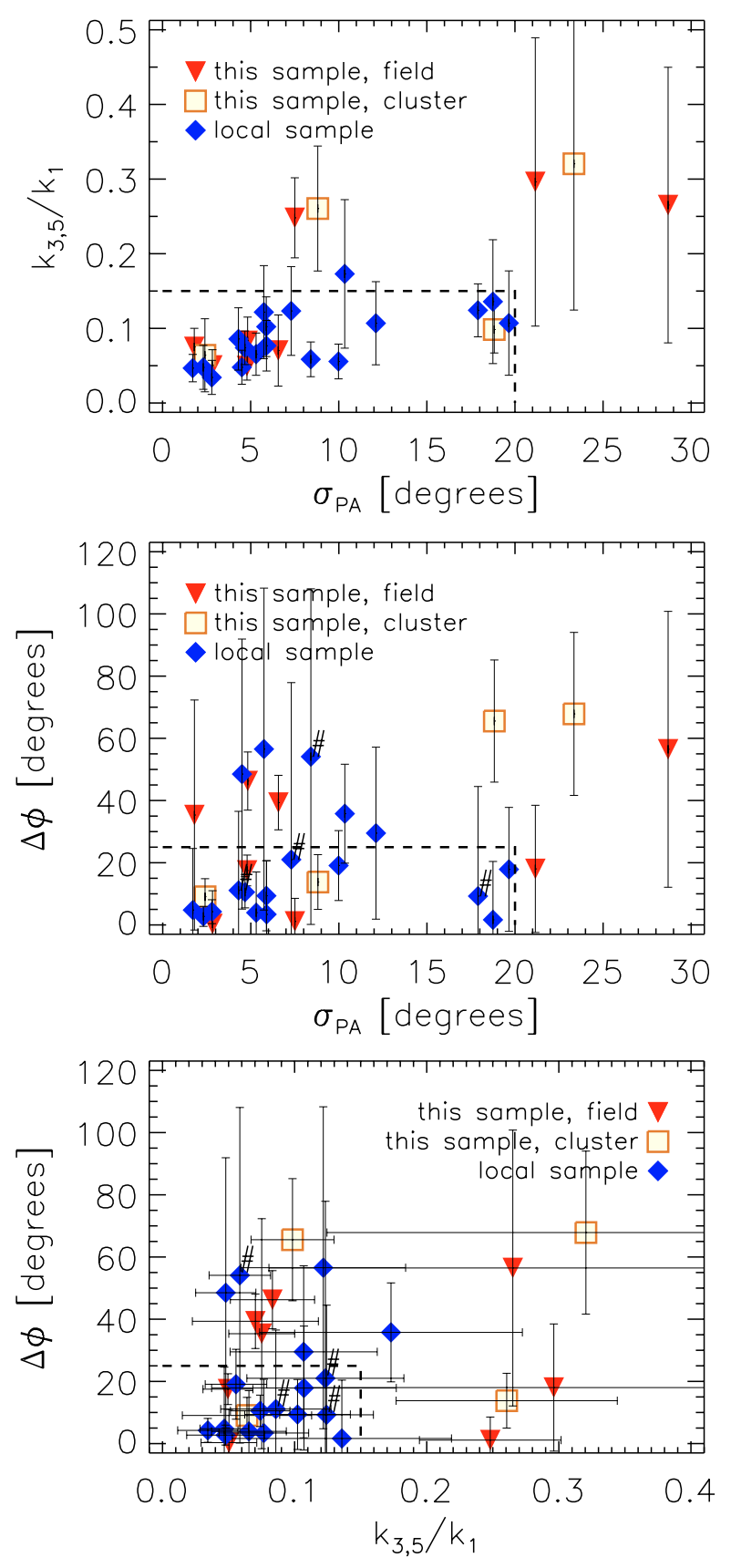

Fig. 11. The same plots as in Fig. 10, now together with the local sample. The boundaries inside which we call the gas kinematics regular are indicated with dashed lines for each parameter. The galaxies that are indicated with \# symbol are the galaxies for which the $\Delta \phi$ is doubtful as explained in Sect. 4.1. Their $\Delta \phi$ measurements are excluded form the analysis.

each galaxy is given in Table 6. We then normalized the ionized gas fluxes to the value of galaxy F7, which has the highest flux (Table 6). The correlation coefficients of the relations between the amount of gas $\left(F_{\mathrm{em}}\right)$ and the irregularity in the gas kinematics are given in Table $4 . \Delta \phi$ and $k_{3,5} / k_{1}$ have an anti-correlation with $F_{\text {em. }}$. These parameters are plotted against each other in Fig. 13.

Using the concentration index, we find no correlation between the galaxy type and the irregularity in the gas kinematics. The amount of gas per unit luminosity is also an indicator of the galaxy type such that later type spirals have more gas and they are less luminous. We find an anti-correlation between this
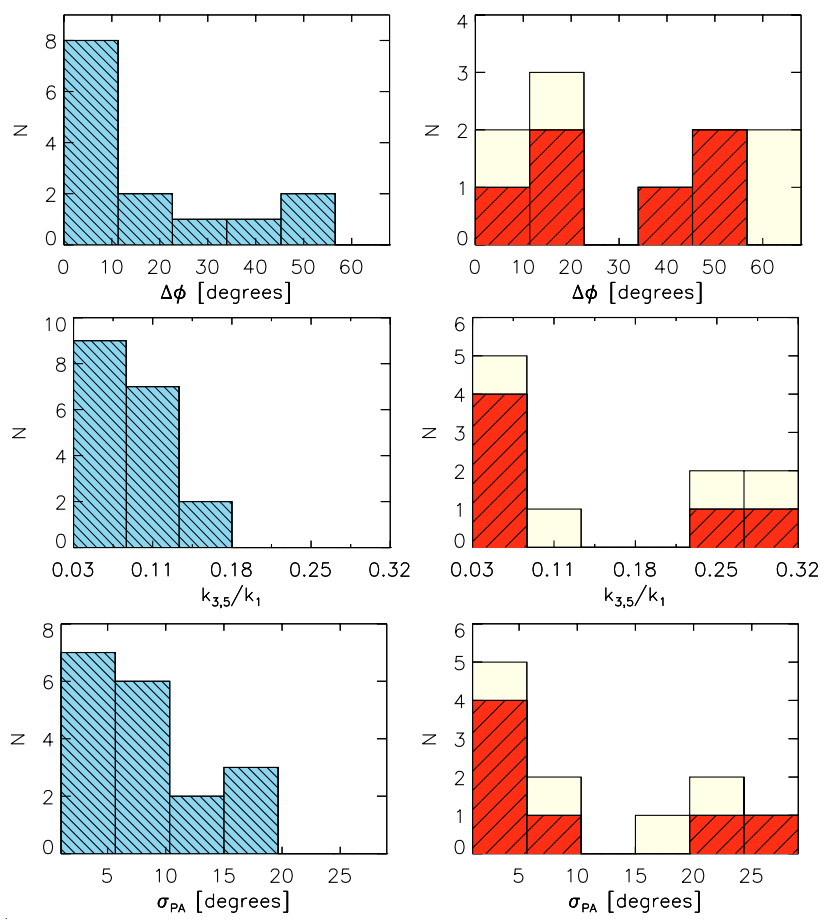

Fig. 12. Histograms of the mean misalignment between kinematic and photometric major axes $(\Delta \phi)$, mean $k_{3,5} / k_{1}$ and standard deviation of kinematic position angle $\left(\sigma_{\mathrm{PA}}\right)$. The local sample is given in blue (the column on the left, fine-shaded at $-45^{\circ}$ ), our sample (the column on the right): field galaxies in red (coarse-shaded at $45^{\circ}$ ) and cluster galaxies in light yellow (not shaded). $\Delta \phi$ of the galaxies that have $\epsilon \leq 0.1$ (galaxy F2 in "this sample", NGC 628, NGC 3184, NGC 3938 and NGC 5713 in the local sample), $k_{3,5}$ of galaxy F5 (this sample) and all parameters for galaxy F10 (this sample) are doubtful as explained in Sect. 4.1. Therefore they are excluded from the histograms. The cluster galaxies are distributed within similar ranges as the field galaxies.

parameter and both $\Delta \phi$ and $\sigma_{\mathrm{PA}}$ (Table 4 , Fig. 13). In all correlations with $F_{\mathrm{em}}$ and $F_{\mathrm{em}} / L_{B}$, one sees that the galaxies with large emission line fluxes generally have very regular velocity fields (Fig. 13). Since the number of objects that have large $F_{\text {em }}$ and $F_{\text {em }} / L_{B}$ values is very small, the correlations we find for these two parameters are most probably spurious.

Galaxies in our sample have redshifts between 0.1 and 0.9. Using both our sample and the local sample together, we checked whether the regularity of the gas kinematics is a function of redshift. In order not to have the bias of the environment on this analysis, we used only the field galaxies. No correlation was found. Including the cluster galaxies in the correlation does not change the result. Since the number of galaxies in our sample is small, this issue should be revisited in the future.

\subsection{Special cases}

In our sample, some galaxies need some special mention: galaxies F2, F5 and F10. Galaxy F2 has a large $\Delta \phi$, even though it has regular kinematics according to the other criteria. It is nearly face-on $(\epsilon=0.1)$ (Fig. B.9a), which causes the photometric position angle measurements to be unreliable. Therefore, $\Delta \phi$ is not meaningful in this case.

For galaxy F5, only the inner part of the galaxy is included (Appendix, Fig. A.12i) for the parameter $k_{3,5} / k_{1}$ due to the large misalignment of the kinematic and photometric axis, so that the range for which it is determined is much smaller than for $\Delta \phi$ and 

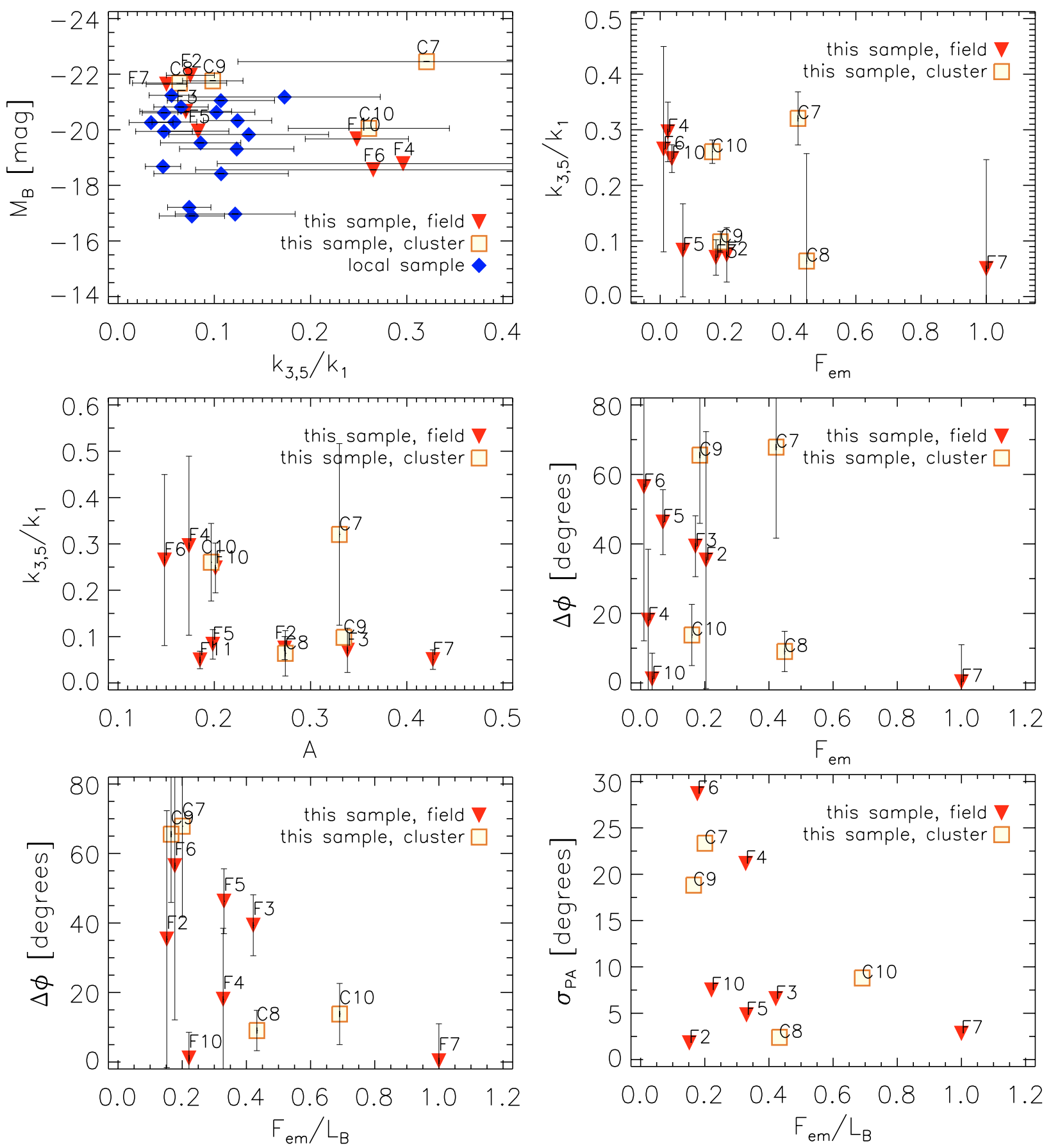

Fig. 13. Top left: $k_{3,5} / k_{1}$ versus absolute magnitude in the $B$ band $\left(M_{B}\right)$. Middle left: asymmetry index (A) versus $k_{3,5} / k_{1}$. Bottom left: relative [OII] 3727 flux per unit $B$ band luminosity $\left(F_{\mathrm{em}} / L_{B}\right)$ versus the mean misalignment between the photometric and the kinematic position angle $(\Delta \phi)$. Top right: $k_{3,5} / k_{1}$ versus the relative [OII] 3727 flux $\left(F_{\mathrm{em}}\right)$. Middle right: mean misalignment between the the photometric and the kinematic position angle $(\Delta \phi)$ versus the relative [OII]3727 flux $\left(F_{\mathrm{em}}\right)$. Bottom right: standard deviation of the kinematic position angle $\left(\sigma_{\mathrm{PA}}\right)$ versus relative [OII] 3727 flux per unit $B$ band luminosity $\left(F_{\text {em }} / L_{B}\right)$.

$\sigma_{\mathrm{PA}}$. There are a few more galaxies that have large misalignments (e.g. galaxy F3 and galaxy C9). In those cases, however, the redshifts are much higher and the radial extent of the observed velocity fields is rather small, so that the measurements of all parameters is limited to the same region.

The data of galaxy F10 are very noisy, so the high order Fourier terms are strongly affected (Fig. B.17e). In our sample we exclude $k_{3,5} / k_{1}$ of galaxy F5, $\Delta \phi$ of the galaxies that have $\epsilon \leq 0.1$ (which is the case only for galaxy F2) and all parameters for galaxy F10 from the analysis.
In the local sample, we excluded the $\Delta \phi$ of the galaxies that have $\epsilon \leq 0.1$ (NGC 628, NGC 3184, NGC 3938, NGC 5713).

\section{Discussion}

Field spirals that have an unbarred morphology and no nearby neighbors are expected to have regular velocity fields (Rubin et al. 1999). However, about 70 percent of the spiral galaxies are expected to have bars (Eskridge et al. 2000; Knapen et al. 2000). A bar and spiral arms may cause large scale deviations 
Table 4. Linear Pearson correlation coefficients.

\begin{tabular}{lccc}
\hline \hline & $\sigma_{\mathrm{PA}}-k_{3,5} / k_{1}$ & $\Delta \phi-\sigma_{\mathrm{PA}}$ & $k_{3,5} / k_{1}-\Delta \phi$ \\
\hline this sample & 0.5 & 0.7 & 0.2 \\
local sample & 0.7 & 0.1 & 0.7 \\
\hline & $R_{\mathrm{d}}-k_{3,5} / k_{1}$ & $R_{\mathrm{d}}-\sigma_{\mathrm{PA}}$ & $R_{\mathrm{d}}-\Delta \phi$ \\
this sample & -0.3 & -0.4 & -0.3 \\
local sample & 0.0 & 0.1 & 0.0 \\
\hline & $M_{B}-k_{3,5} / k_{1}$ & $M_{B}-\sigma_{\mathrm{PA}}$ & $M_{B}-\Delta \phi$ \\
this sample & 0.5 & 0.4 & 0.4 \\
local sample & 0.1 & -0.2 & 0.1 \\
\hline & $A-k_{3,5} / k_{1}$ & $A-\sigma_{\mathrm{PA}}$ & $A-\Delta \phi$ \\
this sample & 0.0 & -0.3 & 0.0 \\
\hline & $C-k_{3,5} / k_{1}$ & $C-\sigma_{\mathrm{PA}}$ & $C-\Delta \phi$ \\
this sample & 0.1 & 0.2 & 0.1 \\
\hline & $F_{\mathrm{em}}-k_{3,5} / k_{1}$ & $F_{\mathrm{em}}-\sigma_{\mathrm{PA}}$ & $F_{\mathrm{em}}-\Delta \phi$ \\
this sample & -0.5 & -0.4 & -0.7 \\
\hline & $F_{\mathrm{em}} / L_{B}-k_{3,5} / k_{1}$ & $F_{\mathrm{em}} / L_{B}-\sigma_{\mathrm{PA}}$ & $F_{\mathrm{em}} / L_{B}-\Delta \phi$ \\
this sample & -0.4 & -0.5 & -0.7 \\
\hline & $z-k_{3,5} / k_{1}$ & $z-\sigma_{\mathrm{PA}}$ & $z-\Delta \phi$ \\
both samples & -0.1 & -0.2 & 0.4 \\
\hline
\end{tabular}

$\Delta \phi$ of the galaxies that have $\epsilon \leq 0.1$ (galaxy F2 in "this sample", NGC 628, NGC 3184, NGC 3938 and NGC 5713 in the local sample), $k_{3,5} / k_{1}$ of galaxy F5 (this sample) and all parameters for galaxy F10 (this sample) are doubtful as explained in Sect. 4.1. Therefore they are excluded while calculating the correlation coefficients. For the calculation of the correlations with the redshift, only field galaxies were used. So the results do not have the bias of the environment. Including the cluster galaxies does not change the result.

Table 5. Irregularity fraction.

\begin{tabular}{lccc}
\hline \hline & frac $_{\sigma_{\mathrm{PA}}}$ & $\mathrm{frac}_{\Delta \phi}$ & frac $_{k_{3,5} / k_{1}}$ \\
& $(1)$ & $(2)$ & $(3)$ \\
\hline field \& cluster & $27 \pm 13 \%$ & $50 \pm 16 \%$ & $40 \pm 15 \%$ \\
only field & $29 \pm 17 \%$ & $50 \pm 20 \%$ & $33 \pm 19 \%$ \\
only cluster & $25 \pm 22 \%$ & $50 \pm 25 \%$ & $50 \pm 25 \%$ \\
\hline
\end{tabular}

Column (1): fraction of irregular velocity fields according to $\sigma_{\mathrm{PA}}$ criterion and its Poisson error; Col. (2): fraction of irregular velocity fields according to $\Delta \phi$ criterion and its Poisson error; Col. (3): fraction of irregular velocity fields according to $k_{3,5} / k_{1}$ criterion and its Poisson error.

Note: $k_{3,5} / k_{1}$ of galaxy F5, $\Delta \phi$ of the galaxies that have $\epsilon \leq 0.1$ (galaxy F2) and all parameters for galaxy F10 have been excluded from the calculations as explained in Sect. 4.1.

from circular rotation. This is discussed in Fridman et al. (2005) together with different possible reasons for small scale anomalies (e.g. nuclear activity, gas outflow from a star formation burst region, a shock excitation mechanism, etc.). See also Wong et al. (2004) and Fathi et al. (2005) where noncircular motions such as bar streaming, inflow and a warp are distinguished from one another using a harmonic decomposition of the velocity fields. However, in the case of distant galaxies, such small scale perturbations would be smeared out in observations, mainly due to seeing (Kronberger et al. 2007).

Interactions between galaxies may cause big deviations from circular rotation (Rampazzo et al. 2005). In clusters of galaxies, several interaction mechanisms can take place in addition to the galaxy-galaxy interactions (Poggianti 2004) such as ram pressure stripping (Gunn \& Gott III 1972; Quilis et al. 2000; Abadi et al. 1999; Roediger \& Brüggen 2007; Kronberger et al. 2008), gas starvation (Bekki et al. 2002; Larson et al. 1980), harassment (Moore et al. 1996; Moore et al. 1998) and viscous stripping (Nulsen 1982). Moran et al. (2007a) give an estimate of where
Table 6. Relative emission line fluxes.

\begin{tabular}{lccc}
\hline \hline $\begin{array}{l}\text { Name } \\
(1)\end{array}$ & $\begin{array}{c}F_{\mathrm{em}} \\
(2)\end{array}$ & $\begin{array}{c}F_{\mathrm{em}} / L_{B} \\
(3)\end{array}$ & $\begin{array}{c}\text { Line } \\
(4)\end{array}$ \\
\hline C7 & 0.42 & 0.20 & {$[\mathrm{OII}] 3727$} \\
C8 & 0.45 & 0.43 & {$[\mathrm{OII}] 3727$} \\
C9 & 0.18 & 0.17 & {$[\mathrm{OII}] 3727$} \\
C10 & 0.16 & 0.69 & {$[\mathrm{OII}] 3727$} \\
F2 & 0.20 & 0.15 & {$[\mathrm{OII}] 3727$} \\
F3 & 0.17 & 0.42 & $\mathrm{H} \beta$ \\
F4 & 0.02 & 0.33 & $\mathrm{H} \beta$ \\
F5 & 0.07 & 0.33 & $\mathrm{H} \beta$ \\
F6 & 0.01 & 0.18 & $\mathrm{H} \alpha$ \\
F7 & 1.00 & 1.00 & {$[\mathrm{OII}] 3727$} \\
F10 & 0.04 & 0.22 & {$[\mathrm{OIII}] 5007$} \\
\hline
\end{tabular}

Column (1): galaxy ID; Col. (2): relative [OII]3727 flux; Col. (3): relative value of [OII] 3727 flux per unit $B$-band luminosity; Col. (4): emission line used to calculate the [OII] 3727 flux for each galaxy.

in MS 0451 different interaction processes are expected to be effective. These phenomena may produce large scale perturbations in the velocity field, similar to the effect of galaxy-galaxy interactions (Rubin et al. 1999). Therefore the fraction of irregular velocity fields is expected to be higher for cluster galaxies than for field galaxies.

We use three indicators of irregular gas kinematics: $\Delta \phi, \sigma_{\mathrm{PA}}$ and $k_{3,5} / k_{1}$ (see Sect. 4). $\sigma_{\mathrm{PA}}$ and $k_{3,5} / k_{1}$ seem to correlate with each other, while this is not the case for all parameters. It is also not expected that all phenomena that may cause kinematical irregularities would increase each parameter. In case of interaction processes, the disturbance in the velocity field might cause all parameters to increase. A secondary component in the velocity field would increase the $k_{3,5} / k_{1}$ term while the components do not have to be kinematically misaligned. The information from all parameters together could be used to interpret what kind of a phenomenon might be responsible from the irregularities in the gas kinematics. However, the resolution effects should be taken into account while doing that (see Appendix A).

We have measured these parameters for the galaxies in our sample and in the SINGS local sample. All local galaxies apart from one cluster member have regular gas kinematics according to the $k_{3,5} / k_{1}$ criterion while all galaxies are regular according to the $\sigma_{\mathrm{PA}}$ criterion. There are four galaxies that do not meet the $\Delta \phi$ criterion (excluding the face-on cases as mentioned in Sect. 4.1). Each galaxy in the local sample that has a large $\Delta \phi$ value is either peculiar or is a cluster member. While some galaxies in our high $z$ sample have $k_{3,5} / k_{1}$ values that are far above the region where the local galaxies are distributed, this is not the case for the $\Delta \phi$ and $\sigma_{\mathrm{PA}}$ parameters (the highest $\sigma_{\mathrm{PA}}$ value in our sample belongs to galaxy $\mathrm{F} 6$ which has a kinematically decoupled core). Galaxies in both samples are distributed within similar $\sigma_{\mathrm{PA}}$ and $\Delta \phi$ ranges. Garrido et al. (2005) reported for their local sample that, on average, the misalignment between the kinematic and the photometric position angle is $15^{\circ} \pm 19^{\circ}$ and the maximum value they measured is $75^{\circ}$. Also in our sample, the fraction of galaxies that are irregular according to the $\Delta \phi$ criterion is the highest. This kind of information might give clues to the dominant phenomena that are responsible for the irregularities or the disturbances that take longer to recover in the gas kinematics. That would only be reliable, however, in case of high resolution data. At low spatial resolution, some combinations of a large $k_{3,5} / k_{1}$ parameter and sampling can cause the measured values of $\sigma_{\mathrm{PA}}$ and $\Delta \phi$ to be boosted (see Appendix A). 
The percentage of galaxies in our sample that have irregular gas kinematics are: $27 \pm 13 \%$ (according to $\sigma_{\mathrm{PA}}$ criterion), $50 \pm 16 \%$ ( $\Delta \phi$ criterion), $40 \pm 15 \%$ ( $k_{3,5} / k_{1}$ criterion) and $58 \pm$ $14 \%$ (any criterion). Among these galaxies, there are 4 cluster members and they all are closer than $1 \mathrm{Mpc}$ to the center. Since this is well inside the virial radius of the cluster, we expect the ICM density to be rather high and, therefore, able to distort the ISM of the galaxy disks. On the other hand, relative velocities are quite high so that direct encounters would have rather negligible effects. When we analyze the distributions of each of the three parameters (Fig. 12), we see that cluster and field galaxies have similar behaviour.

Flores et al. (2006) made a kinematical classification based on the alignment of the photometric and kinematic axes and the agreement between the peak of their $\sigma$ map and the kinematic center. They found that $35 \%$ of the disks are rotating and as such regular, for a sample of 35 galaxies at $0.4<z<0.75$. For the galaxies in our sample that are in the same redshift interval as Flores et al.'s sample, (7 galaxies, but we excluded the special cases mentioned in Sect. 4.1 from the analysis) the fraction of objects that have regular gas kinematics is: $40 \pm 22 \%$ (according to $\Delta \phi$ criterion), $83 \pm 15 \%$ ( $\sigma_{\mathrm{PA}}$ criterion) and $67 \pm 19 \%$ ( $k_{3,5} / k_{1}$ criterion). So, using the $\Delta \phi$ criterion, our result is comparable with their result. Förster Schreiber et al. (2006) investigated galaxy kinematics at a much higher redshift. They obtained $\mathrm{H} \alpha$ maps of 14 massive star forming galaxies at $z \approx 2-3$. Four of the six best spatially resolved cases match the expectations for a simple rotating disk. Shapiro et al. (2008) introduce a method to distinguish merging and non-merging systems. Using kinemetry, they measure the deviation from the ideal case for both the gas velocity field and the stellar continuum intensity map of the galaxies. They use these parameters of 29 merging and regular template galaxies to define a criterion that discerns between the two. They then apply this method to $11 z \approx 2$ galaxies and find that $>50 \%$ of these systems are consistent with a single rotating disk interpretation. Considering this result together with high (up to $100 M_{\odot} \mathrm{yr}^{-1}$ ) star formation rates of these systems, they argue that the smooth accretion mechanism can play an important role in the early stages of the massive-galaxy evolution.

Using $\mathrm{H} \alpha$ velocity fields of local galaxies, Garrido et al. (2005) found that the asymmetry of the rotation curves (or lopsidedness) is correlated with the morphological type, the luminosity, the $(B-V)$ colour and the maximal rotational velocity of galaxies. They find that brighter, more massive and redder galaxies have smaller deviations from regular kinematics. Here we do not use a parameter that corresponds to kinematic lopsidedness. Although we know that lopsidedness would cause $k_{3,5} / k_{1}$ to increase, large $k_{3,5} / k_{1}$ values are not necessarily due to lopsidedness (e.g. secondary kinematical component can also do the job). Garrido et al. (2005) also measured the misalignment between the kinematic and photometric position angles of their galaxies and found no correlation with morphological type. We investigated this effect using two indicators of galaxy type: the concentration index and the amount of gas per unit luminosity. We do not find a correlation with the concentration index while we do find a negative correlation using $F_{\mathrm{em}} / L_{B}$ which means that later type galaxies have smaller misalignments. This correlation could be spurious as explained in Sect. 4.1.

We do not find a correlation between the photometric asymmetry and irregularity in the gas kinematics. The asymmetry index indicates the inhomogenity in the $I$-band light distribution. This parameter is a function of the galaxy type. For the $\mathrm{E} / \mathrm{S} 0 \mathrm{~s}$ it is the smallest, it becomes higher for spirals and it is the highest for irregular galaxies. Apart from the intrinsic asymmetry of a galaxy, interaction processes or mergers can also cause the asymmetry index to increase. In case of an interaction process that is strong enough to affect the distribution of the stars, it is expected that the gas component is also disturbed. On the other hand, the phenomena that cause irregularities in the gas kinematics do not necessarily affect the stellar component since it is gravitationally more stable. There is one case in our sample for which both the photometry and the kinematics are irregular (galaxy C9). It has $\Delta \phi=66 \pm 20$, and it is regular according to the other criteria $\left(\sigma_{\mathrm{PA}}\right.$ and $\left.k_{3,5} / k_{1}\right)$.

\section{Summary and conclusions}

In this paper we present the kinematic and photometric analysis of 22 galaxies, 11 of which are members of MS 0451 at $z=0.54$. All cluster members are within $2 \mathrm{Mpc}$ from the center and most of them are closer than $1 \mathrm{Mpc}$. Among the cluster objects, there are two in interaction with each other and 5 with weak or no emission. For 8 field and 6 cluster galaxies we were able to obtain the gas velocity fields. We used kinemetry to characterise these velocity fields using Fourier coefficients. The maximum radius for which we could measure the kinematic parameters (position angle, ellipticity, etc.) of each galaxy is given in Table 2.

We use 3 parameters to quantify the regularity of the velocity fields: $k_{3,5} / k_{1}, \sigma_{\mathrm{PA}}$ and $\Delta \phi$ (Sect. 4$)$ and introduce a criterion for each parameter to distinguish between regular and irregular gas kinematics. To do that, we also measured each parameter for nearby galaxies in the SINGS survey and investigated the distribution of these galaxies in the parameter space. The regularity criteria we define are: $\Delta \phi \leq 25, \sigma P A \leq 20$ and $k_{3,5} / k_{1} \leq 0.15$.

We calculated the fraction of galaxies in our sample that have irregular gas kinematics according to each criterion (without subdividing as field galaxies and cluster members). $27 \pm$ $13 \%$ (according to $\sigma_{\mathrm{PA}}$ criterion), $50 \pm 16 \%$ ( $\Delta \phi$ criterion), $40 \pm$ $15 \%\left(k_{3,5} / k_{1}\right.$ criterion) and $58 \pm 14 \%$ (any criterion). Among these objects, there are four cluster galaxies for which we were able to measure these parameters. We find that field and cluster galaxies span similar range of parameter values (Fig. 12). In particular, we find irregular cases in both environmental classes: $29 \pm 17 \% / 25 \pm 22 \%$ (according to $\sigma_{\mathrm{PA}}$ criterion), $50 \pm 20 \% / 50 \pm$ $25 \%$ ( $\Delta \phi$ criterion), $33 \pm 19 \% / 50 \pm 25 \%\left(k_{3,5} / k_{1}\right.$ criterion $)$ and $50 \pm 18 \% / 75 \pm 22 \%$ (any criterion). However, we note that the number of galaxies we have analyzed is not statistically sufficient to give robust results. In a future paper we will analyse a much larger sample in the same way, increasing the statistics considerably.

We investigated the spatial resolution effects on our kinematic analysis and found that the fraction of irregular velocity fields in a sample determined from low spatial resolution data is an underestimate of the real fraction. We also found out that for some combinations of a large $k_{3,5} / k_{1}$ and sampling, the measured values of $\Delta \phi$ and $\sigma_{\mathrm{PA}}$ will be boosted, so that the different types of irregularity become degenerate in the rebinned data. Hence, identifying irregular galaxies without distinguishing between the three types of irregularity gives more reliable results in case of low spatial resolution.

6 galaxies ( 5 of them are cluster members) have strong absorption lines (mainly CaII H, K and Balmer lines) which enabled us to derive their stellar rotation curves. For three of them (C5, C7 and C9), stellar and gas curves show different behaviour. These are galaxies that are classified to have irregular gas 
kinematics (C5 is interacting with its companion). C7 and C9 also have relatively large photometric asymmetries.

We have performed a surface photometry analysis of each galaxy (Table C.2), and calculated their rest-frame absolute $B$ band magnitude (Table C.1). We also measured some other photometric properties (e.g. asymmetry and concentration index) and investigated the existence of a correlation of these parameters with the kinematic regularity. Although we do not find any significant correlation, these should be re-investigated with a larger sample.

Acknowledgements. We are thankful to the authors of Daigle et al. (2006) for kindly providing us with the $\mathrm{H} \alpha$ velocity fields of the galaxies in the SINGS local sample, Laurent Chemin for the $\mathrm{H} \alpha$ velocity field of NGC 4254 and Davor Krajnović for the Kinemetry software. We thank the anonymous referee for helpful comments and Isabel Pérez Martin for fruitful discussion. We appreciate the efficient support of ESO and the Paranal staff. This work has been financially supported by the Volkswagen Foundation (I/76520), the Deutsche Forschungsgemeinschaft, DFG (project number ZI 663/6) within the Priority Program 1177, the Kapteyn Astronomical Institute of the University of Groningen and the German Space Agency DLR (project numbers 50 OR 0602, 50 OR 0404, and 50 OR 0301). This research has made use of the NASA/IPAC Extragalactic Database (NED) which is operated by the Jet Propulsion Laboratory, California Institute of Technology, under contract with the National Aeronautics and Space Administration. HST/ACS images were retrieved from the ST-ECF archive.

\section{References}

Abadi, M. G., Moore, B., \& Bower, R. G. 1999, MNRAS, 308, 947

Abraham, R. G., Valdes, F., Yee, H. K. C., \& van den Bergh, S. 1994, ApJ, 432, 75

Andersen, D. R., Bershady, M. A., Sparke, L. S., et al. 2006, ApJS, 166, 505

Arribas, S., Mediavilla, E., Garcia-Lorenzo, B., \& Del Burgo, C. 1997, AJ, 490, 227

Böhm, A., Ziegler, B. L., Saglia, R. P., et al. 2004, A\&A, 420, 97

Bamford, S. P., Milvang-Jensen, B., Aragón-Salamanca, A., \& Simard, L. 2005, MNRAS, 361, 109

Bamford, S. P., Aragón-Salamanca, A., \& Milvang-Jensen, B. 2006, MNRAS, 366, 308

Bedin, L. R., Cassisi, S., Castelli, F., et al. 2005, MNRAS, 357, 1038

Bekki, K., Couch, W. J., \& Shioya, Y. 2002, ApJ, 577, 651

Bertin, E., \& Arnouts, S. 1996, A\&AS, 117, 393

Blanton, M. R., \& Roweis, S. 2007, AJ, 133, 734

Böhm, A., \& Ziegler, B. L. 2007, ApJ, 668, 846

Boomsma, R., Oosterloo, T. A., Fraternali, F., van der Hulst, J. M., \& Sancisi, R. 2005, in Extra-Planar Gas, ed. R. Braun, ASP Conf. Ser., 331, 247

Bower, R. G. 1991, MNRAS, 248, 332

Cappellari, M., \& Emsellem, E. 2004, PASP, 116, 138

Chemin, L., Balkowski, C., Cayatte, V., et al. 2006, MNRAS, 366, 812

Conselice, C. J., Bershady, M. A., \& Jangren, A. 2000, ApJ, 529, 886

Conselice, C. J., Bundy, K., Ellis, R. S., et al. 2005, ApJ, 628, 160

Daigle, O., Carignan, C., Amram, P., et al. 2006, MNRAS, 367, 469

Donahue, M. 1996, ApJ, 468, 79

Dumas, G., Mundell, C. G., Emsellem, E., \& Nagar, N. M. 2007, MNRAS, 379, 1249

Ellingson, E., Yee, H. K. C., Abraham, R. G., Morris, S. L., \& Carlberg, R. G. 1998, ApJS, 116, 247

Eskridge, P. B., Frogel, J. A., Pogge, R. W., et al. 2000, AJ, 119, 536

Falcón-Barroso, J., Bacon, R., Bureau, M., et al. 2006, MNRAS, 2, 529

Fathi, K., van de Ven, G., Peletier, R. F., et al. 2005, MNRAS, 364, 773

Flores, H., Hammer, F., Puech, M., Amram, P., \& Balkowski, C. 2006, A\&A, 455, 107

Förster Schreiber, N. M., Genzel, R., Lehnert, M. D., et al. 2006, ApJ, 645, 1062
Fridman, A. M., Afanasiev, V. L., Dodonov, S. N., et al. 2005, A\&A, 430, 67 Ganda, K., Falcón-Barroso, J., Peletier, R. F., et al. 2006, MNRAS, 367, 46 Garrido, O., Marcelin, M., Amram, P., et al. 2005, MNRAS, 362, 166 Gunn, J. E., \& Gott III, J. R. 1972, ApJ, 176, 1

Jäger, K., Ziegler, B. L., Böhm, A., et al. 2004, A\&A, 422, 907

Kapferer, W., Kronberger, T., Schindler, S., Böhm, A., \& Ziegler, B. L. 2006, A\&A, 446, 847

Kassin, S. A., Weiner, B. J., Faber, S. M., et al. 2007, ApJ, 660, L35

Kay, S. T., da Silva, A. C., Aghanim, N., et al. 2007, MNRAS, 377, 317

Knapen, J. H., Shlosman, I., \& Peletier, R. F. 2000, ApJ, 529, 93

Koopmann, R. A., \& Kenney, J. D. P. 2004, ApJ, 613, 866

Krajnović, D., Cappellari, M., de Zeeuw, P. T., \& Copin, Y. 2006, MNRAS, 366, 787

Kronberger, T., Kapferer, W., Schindler, S., et al. 2006, A\&A, 458, 69

Kronberger, T., Kapferer, W., Schindler, S., \& Ziegler, B. L. 2007, A\&A, 473, 761

Kronberger, T., Kapferer, W., Ferrari, C., Unterguggenberger, S., \& Schindler, S. 2008, ArXiv e-prints, 801

Landolt, A. U. 1992, AJ, 104, 340

Larson, R. B., Tinsley, B. M., \& Caldwell, C. N. 1980, ApJ, 237, 692

Menanteau, F., Ford, H. C., Motta, V., et al. 2006, AJ, 131, 208

Mendes de Oliveira, C., Amram, P., Plana, H., \& Balkowski, C. 2003, AJ, 126, 2635

Metevier, A. J., Koo, D. C., Simard, L., \& Phillips, A. C. 2006, ApJ, 643, 764

Moore, B., Katz, N., Lake, G., Dressler, A., \& Oemler, A. 1996, Nature, 379, 613

Moore, B., Lake, G., \& Katz, N. 1998, ApJ, 495, 139

Moran, S. 2008, California Institute of Technology

Moran, S. M., Ellis, R. S., Treu, T., et al. 2007a, ApJ, 671, 1503

Moran, S. M., Miller, N., Treu, T., Ellis, R. S., \& Smith, G. P. 2007b, ApJ, 659, 1138

Nakamura, O., Aragón-Salamanca, A., Milvang-Jensen, B., et al. 2005, MNRAS, 366, 144

Noordermeer, E., van der Hulst, J. M., Sancisi, R., Swaters, R. S., \& van Albada, T. S. 2007, MNRAS, 376, 1513

Nulsen, P. E. J. 1982, MNRAS, 198, 1007

Peng, C. Y., Ho, L. C., Impey, C. D., \& Rix, H. 2002, AJ, 124, 266

Poggianti, B. M. 2004, in Baryons in Dark Matter Halos, ed. R. Dettmar, U. Klein, \& P. Salucci

Quilis, V., Moore, B., \& Bower, R. 2000, Science, 288, 1617

Rampazzo, R., Plana, H., Amram, P., et al. 2005, MNRAS, 356, 1177

Roediger, E., \& Brüggen, M. 2007, MNRAS, 380, 1399

Rubin, V. C., Hunter, D. A., \& Ford, W. K. J. 1991, ApJS, 76, 153

Rubin, V. C., Waterman, A. H., \& Kenney, J. D. P. 1999, AJ, 118, 236

Sánchez-Blázquez, P., Peletier, R. F., Jiménez-Vicente, J., et al. 2006, MNRAS, 371,703

Sarzi, M., Falcón-Barroso, J., Davies, R. L., et al. 2006, MNRAS, 366, 1151

Schink, H., Nicklas, H. E., Harke, R., et al. 2000, in Optical and IR Telescope Instrumentation and Detectors, ed. M. Iye, \& A. F. Moorwood, Proc. SPIE, 4008, 175

Schlegel, D. J., Finkbeiner, D. P., \& Davis, M. 1998, ApJ, 500, 525

Shapiro, K. L., Genzel, R., Forster Schreiber, N. M., et al. 2008 [arXiv:0802.0879]

Sofue, Y., \& Rubin, V. 2001, ARA\&A, 39, 137

Stanford, S. A., Eisenhardt, P. R., Dickinson, M., Holden, B. P., \& De Propris, R. 2002, ApJS, 142, 153

Swinbank, A. M., Bower, R. G., Smith, G. P., et al. 2006, MNRAS, 368, 1631

Tonry, J. L., Schmidt, B. P., Barris, B., et al. 2003, ApJ, 594, 1

Tully, R. B., \& Fisher, J. R. 1977, A\&A, 54, 661

van Gorkom, J. H. 2004, in Clusters of Galaxies: Probes of Cosmological Structure and Galaxy Evolution, ed. J. S. Mulchaey, A. Dressler, \& A. Oemler, 305

Wong, T., Blitz, L., \& Bosma, A. 2004, ApJ, 605, 183

Wright, E. L. 2006, PASP, 118, 1711

Ziegler, B. L., Böhm, A., Fricke, K. J., et al. 2002, ApJ, 564, L69

Ziegler, B. L., Böhm, A., Jäger, K., Heidt, J., \& Möllenhoff, C. 2003, ApJ, 598, L87 


\section{Appendix A: Resolution Effects on the Kinematic Analysis}

More than half of our galaxies are at redshifts above 0.5 and were observed with pixels of $1^{\prime \prime} \times 0.25$ and a seeing of 0.76 $(F W H M)$. Here we test how much our kinematical analysis depends on these observing conditions. For that, we simulate the combined effect of seeing and spatial resolution on the observed velocity field, as a function of distance to the observed galaxy. We start with the velocity field of a galaxy at distance $d$ with a pixel size of $m \times 4 m$. To represent the observed velocity field of this galaxy when it is $N$ times further away, we first convolve it with a Gaussian psf:

$$
F W H M_{\text {new }}=\sqrt{\left(N \times F W H M_{\text {current }}\right)^{2}-F W H M_{\text {current }}^{2}}
$$

where $F W H M_{\text {current }}$ is the $F W H M$ of the point spread function of the velocity field at the distance $d$. After the convolution process, we rebin the velocity field to have a pixel size of $\mathrm{Nm} \times 4 \mathrm{Nm}$. To calculate the velocity corresponding to the new pixel, we measure the intensity weighted velocity within its borders. We applied this method to several velocity fields that have different types and levels of irregularity. These include a regular $\mathrm{H} \alpha$ velocity field (Fig. A.1a), simulated velocity fields of interacting galaxies (Kronberger et al. 2007) (Figs. A.1b-d), warped models that have simple rotation along each orbit (Figs. A.1e-g) and some models made by adding 3rd and 5th order Fourier terms with different amplitudes to the velocity field of a simple rotating disk (Figs. A.1h-m). The warped velocity fields were made to measure the resolution effects on the $\sigma_{\mathrm{PA}}$ and $\Delta \phi$ parameters while the last group of models were made to evaluate the resolution effects on the $k_{3,5} / k_{1}$ term.

For the intensity weighting of galaxy 1 , we used its $\mathrm{H} \alpha$ image. For galaxies 2-4, we used their stellar mass density maps assuming that they have constant mass to light ratios. For the rest of the models, we used an intensity map that has the same orientation and ellipticity as the corresponding velocity field and is in the form of

$I(r)=\frac{A}{\left(1+\frac{r}{B}\right)^{2}}$,

where $A$ and $B$ are constants that are chosen to make an intensity profile that is similar to the $\mathrm{H} \alpha$ intensity profile of galaxy 1 . $r$ is the distance to the center of the map. The velocity field of galaxy 1 calculated at several distances is shown in Fig. A.2. For the other galaxies, we show only the velocity field at the largest simulated distance, in Fig. A.1. The velocity maps in these figures show the velocity corresponding to each pixel at its center. The values in between the pixel centers were visualized using linear interpolation.

The velocity fields that are used for this exercise do not all have the same initial distance and resolution. However, they all have similar grid sizes with our observations at their largest simulated distance. This better indicates how comparable the simulations are with our observations than the actual resolution. Because our sample is not homogenius in size and redshift, but all galaxies were observed with 3 pixels along their minor axis. For the redshift of our cluster galaxies $(z=0.54)$, the physical size of our pixels is $\approx 1.6 \times 6.4 \mathrm{kpc}$. For a magnification of 25.6 , the range of our simulations, the pixel size in the local universe would be $63 \times 250 \mathrm{pc}$. This would correspond to an angular resolution of $\approx 0$ ' $85 \times 3$ '. 4 at the distance of Virgo, somewhat larger than can be obtained with current ground-based telescopes in the optical. For the velocity fields of galaxies $2-4$, the intrinsic resolution was so low, that we could only degrade them by a factor 8 . For the rest the maximum factor was 25 .

For each velocity field simulated at several resolutions, we measured the (ir)regularity parameters $\left(\sigma_{\mathrm{PA}}, \Delta \phi\right.$ and $\left.k_{3,5} / k_{1}\right)$. In Fig. A.3 we plot $k_{3,5} / k_{1}$ as a function of distance for galaxies that have $k_{3,5} / k_{1}$ greater and less than 0.15 , to avoid overcrowding of the plots. For galaxies that have a large intrinsic value of $k_{3,5} / k_{1}$, the value that is recovered at low resolution is always smaller except in a few cases. For some galaxies, a decrease in the resolution causes an increase in this parameter as a result of a different gradient pattern introduced by intensity weighted average velocities within larger pixels. This is the case for galaxies 8 and 13. For a few galaxies, $k_{3,5} / k_{1}$ decreases substantially with decreasing resolution, e.g. galaxy 4 . Regular velocity fields show a regular, slow decrease, which is accompanied by a small deviation in some cases. Overall, small values of $k_{3,5} / k_{1}$ measured for galaxies at large distances do not provide information about their intrinsic values, while large measured values indicate large intrinsic values. There are three cases where the measured $k_{3,5} / k_{1}$ at the largest distance of simulations is above the irregularity treshold (galaxies 2,8 and 13) and they all have intrinsic values that are higher than the treshold. However, only $43 \pm 19 \%$ of the velocity fields that have intrinsic values of $k_{3,5} / k_{1}$ above the irregularity threshold would be identified as such at the lowest resolution. This means that using $k_{3,5} / k_{1}$ as an indicator, a lower limit is found to the number of irregular galaxies.

In Fig. A.4, we plot $\Delta \phi$ and $\sigma_{\mathrm{PA}}$ as a function of distance for the same groups of galaxies as in Fig. A.3 separately. We also give the position angle as a function of radius for both the original velocity field and the velocity field at the largest distance in Fig. A.5. While most galaxies show a continuous decrease of these two parameters with increasing distance, some of the galaxies that have a large $k_{3,5} / k_{1}$ parameter show a strongly varying behavior. The reason for this is the same fact causing the deviation in $k_{3,5} / k_{1}$ parameter itself as explained above. The increase in the $\Delta \phi$ and $\sigma_{\mathrm{PA}}$ terms at lower resolution does not only depend on the $k_{3,5} / k_{1}$ parameter, but also on the gradient structure of the velocity field and how the pixels are positioned on it. At low resolution, $\Delta \phi$ values of galaxy 12 and 13 are measured to be larger than the irregularity treshold although their intrinsic values are around zero. $\sigma_{\mathrm{PA}}$ of galaxies 9 and 10 also go above the irregularity treshold at low resolution although their intrinsic values are below 10. Among these galaxies, all but galaxy 10 have large $k_{3,5} / k_{1}$ parameters (for galaxy $10, k_{3,5} / k_{1}=0.08$ ). This means that in the case of low spatial resolution, the combined effect of a large $k_{3,5} / k_{1}$ and the pixel positions will cause $\Delta \phi$ and $\sigma_{\mathrm{PA}}$ to be larger than their intrinsic values. For galaxies that have a large intrinsic value of $\Delta \phi$ and $\sigma_{\mathrm{PA}}$, the fraction of information that is lost with decreasing resolution is generally higher. In case of galaxies that do not have large $k_{3,5} / k_{1}$ values, $\Delta \phi$ does not change much with resolution. $75 \% \pm 22 \%$ of the velocity fields that are irregular according to the $\Delta \phi$ criterion could be identified at the lowest resolution case. There are 7 velocity fields that are irregular according to the $\sigma_{\mathrm{PA}}$ criterion and $43 \% \pm$ $19 \%$ of them could be identified at the lowest resolution case.

The results of this test show that the fraction of irregular galaxies in a sample determined from low spatial resolution data will be underestimated. A combination of a high $k_{3,5} / k_{1}$ and pixel positioning can cause the measured values of $\Delta \phi$ and $\sigma_{\mathrm{PA}}$ to be boosted, so that the different types of irregularity become degenerate in the rebinned data. Hence, identifying irregular galaxies without distinguishing between the three types of irregularity gives more reliable results in case of low spatial resolution. 
E. Kutdemir et al.: Internal kinematics of spiral galaxies in distant clusters, Online Material p 2
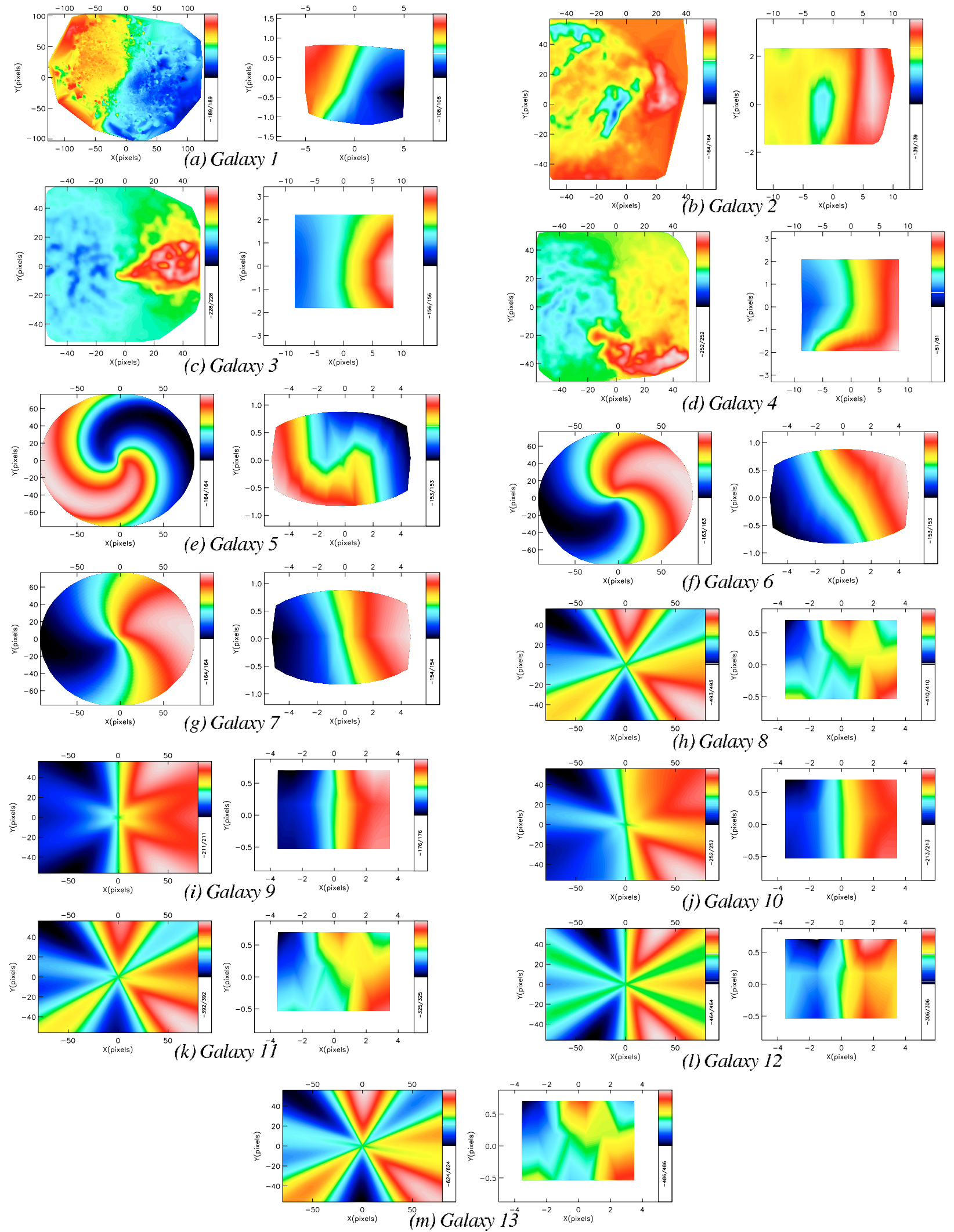

Fig. A.1. a) $\mathrm{H} \alpha$ velocity field of NGC 4254 (galaxy 1) at distance $d$ with pixel size of $m \times m$ and at distance $25.6 d$ with pixel size of $16 m \times 64 m$ respectively. b)-d) Simulated irregular velocity fields: galaxy 2, galaxy 3 and galaxy 4, each at distance $d$ with pixel size of $m \times m$ and at distance $8 d$ with pixel size of $4 m \times 16 m$ respectively. e)-g) Warped disk models with simple rotation along each orbit: galaxy 5 , galaxy 6 and galaxy 7 at distance $d$ with pixel size of $m \times m$ and at distance $25.6 d$ with pixel size of $16 m \times 64 m$ respectively. h) $-\mathbf{m}$ ) 3rd and/or 5th order Fourier terms with different amplitudes added to a simple rotating disk model: galaxy 8, galaxy 9 and galaxy 10, galaxy 11, galaxy 12 and galaxy 13 at distance $d$ with pixel size of $m \times m$ and at distance $25.6 d$ with pixel size of $16 m \times 64 m$ respectively. 
E. Kutdemir et al.: Internal kinematics of spiral galaxies in distant clusters, Online Material p 3

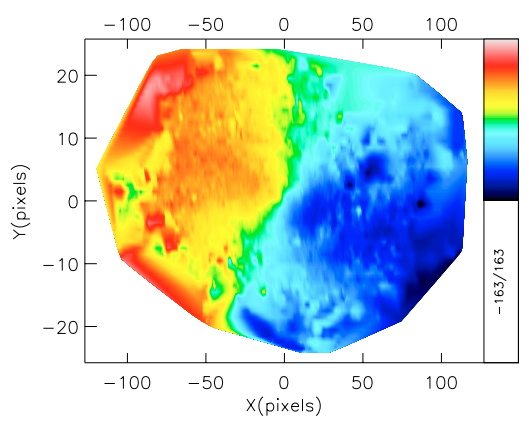

(a)

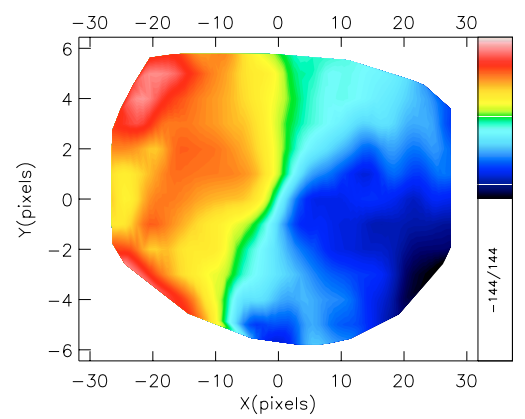

(d)

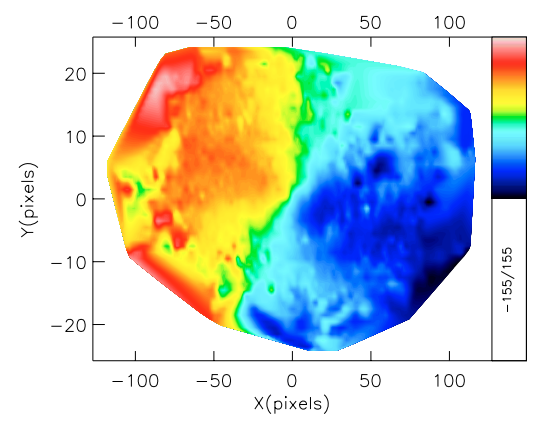

(b)

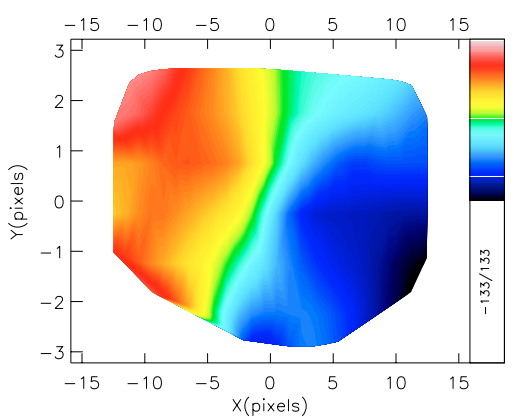

(e)

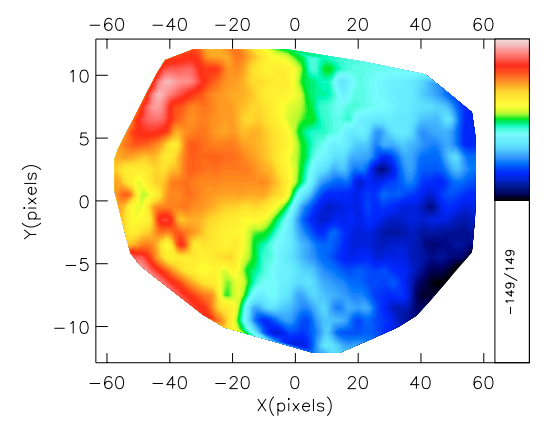

(c)

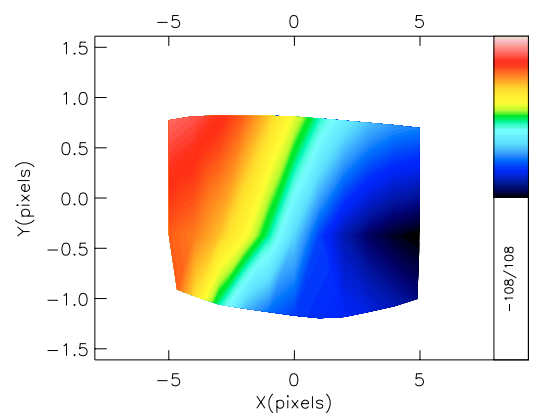

$(f)$

Fig. A.2. Galaxy 1 a) at distance $d$ with $m \times 4 m$ pixel size. b)-f) At distance $1.6 d, 3.2 d, 6.4 d, 12.8 d$ and $25.6 d$ with pixel sizes of $m \times 4 m, 2 m \times$ $8 m, 4 m \times 16 m, 8 m \times 32 m, 16 m \times 64 m$ respectively.

At the lowest resolution, $60 \% \pm 15 \%$ of all irregular galaxies is classified as such according to at least one of our irregularity criteria, while all three regular cases were correctly identified. $90 \% \pm 10 \%$ of the cases that are identified to be irregular at the lowest resolution were intrinsically irregular.
We also checked how the global kinematic position angle and flattening change as a function of the distance. This is given in Fig. A.6. The global position angle is very robust while this is much less the case for the ellipticity. 
E. Kutdemir et al.: Internal kinematics of spiral galaxies in distant clusters, Online Material p 4
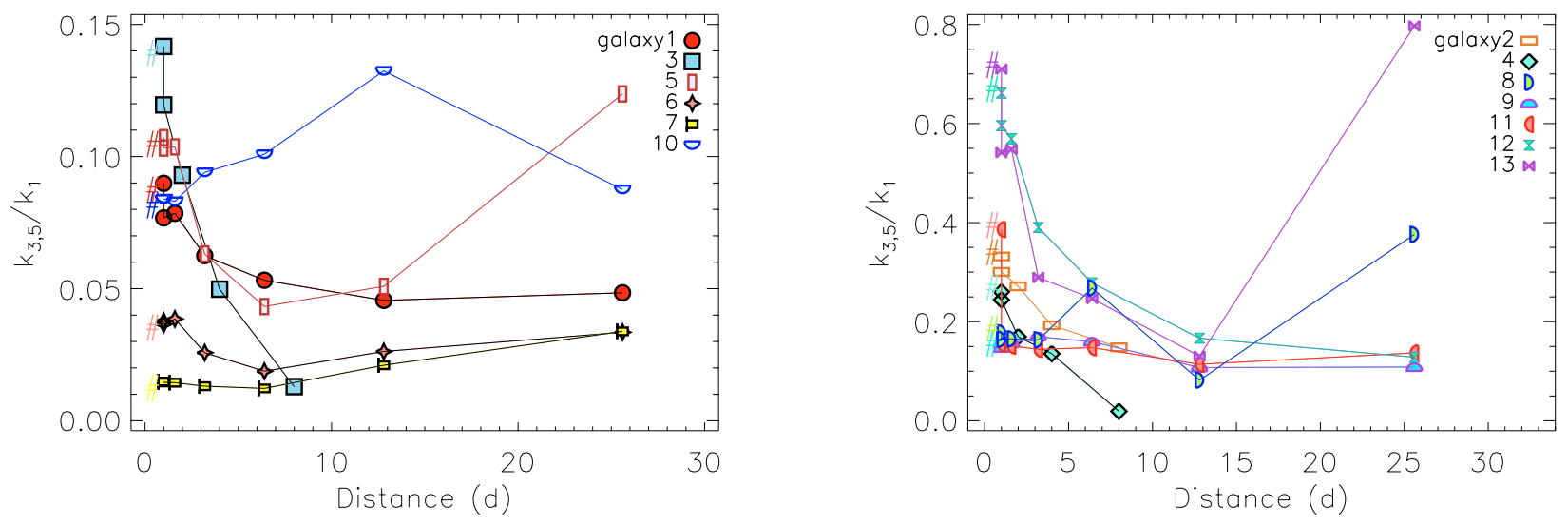

Fig. A.3. $k_{3,5} / k_{1}$ as a function of the distance. The measurements for the original velocity fields are also given in these plots. They are indicated with \# symbol to be able to distinguish them from the velocity field at the same distance with the same pixel size along the $x$ axis and 4 times as large pixel size along the $y$ axis. Left: galaxies that have $k_{3,5} / k_{1}$ less than 0.15 . Right: galaxies that have $k_{3,5} / k_{1}$ greater than 0.15 .

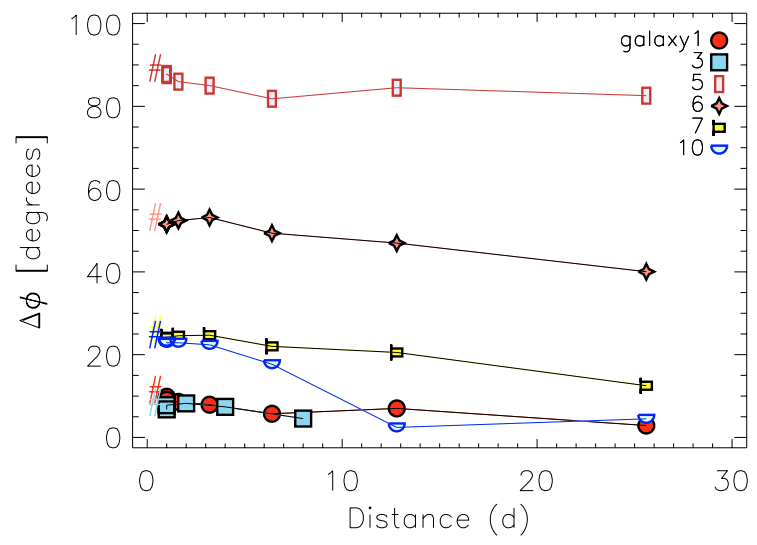

(a)

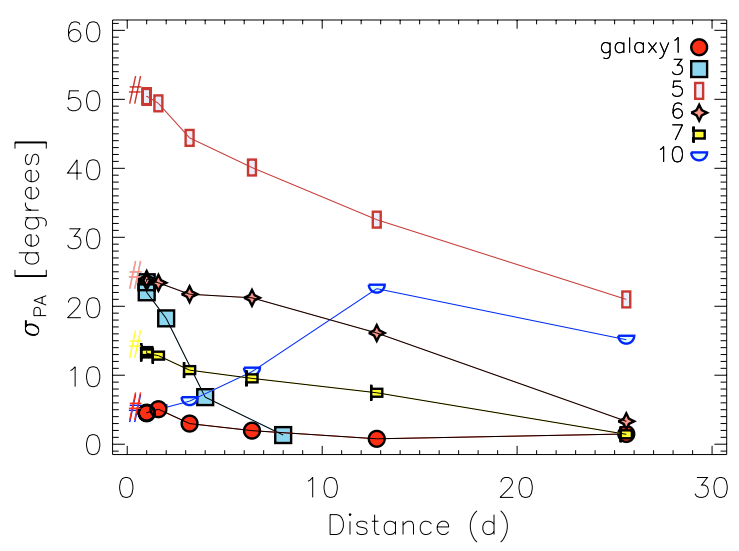

(c)

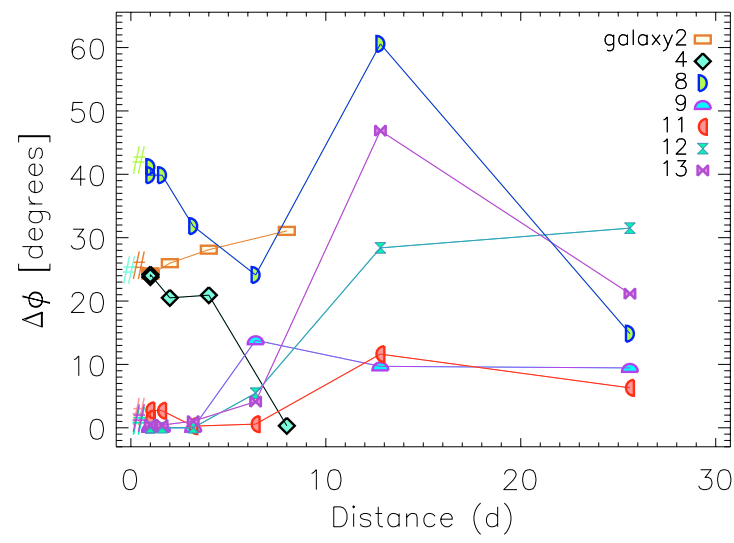

(b)

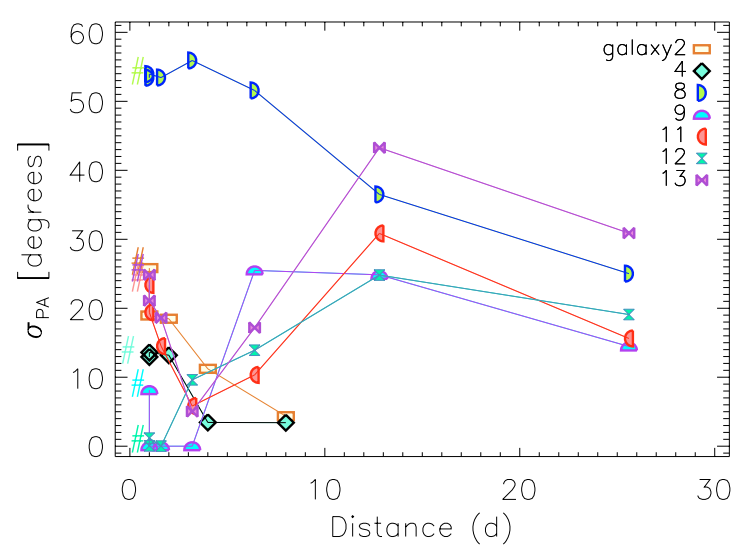

(d)

Fig. A.4. $\Delta \phi$ and $\sigma_{\mathrm{PA}}$ as a function of the distance. The measurements for the original velocity fields are also given in these plots. They are indicated with \# symbol to be able to distinguish them from the velocity field at the same distance with the same pixel size along the $x$ axis and 4 times as large pixel size along the $y$ axis. a) $\Delta \phi$ as a function of the distance for galaxies that have $k_{3,5} / k_{1}$ less than 0.15 . b) $\Delta \phi$ as a function of the distance for galaxies that have $k_{3,5} / k_{1}$ greater than 0.15 . c) $\sigma_{\mathrm{PA}}$ as a function of the distance for galaxies that have $k_{3,5} / k_{1}$ less than 0.15 . d) $\sigma_{\mathrm{PA}}$ as a function of the distance for galaxies that have $k_{3,5} / k_{1}$ greater than 0.15 . 
E. Kutdemir et al.: Internal kinematics of spiral galaxies in distant clusters, Online Material p 5

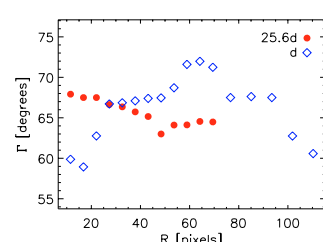

(a) Galaxy 1

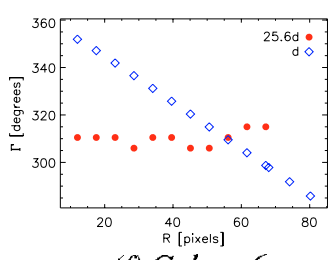

(f) Galaxy 6

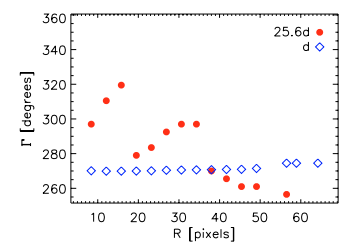

(k) Galaxy 11

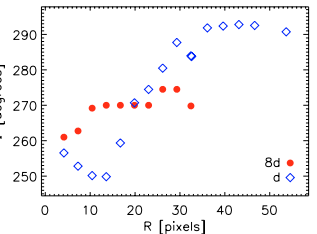

(b) Galaxy 2

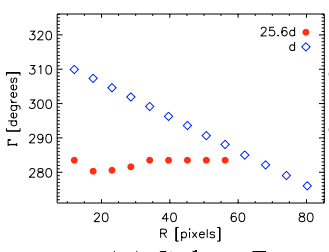

(g) Galaxy 7

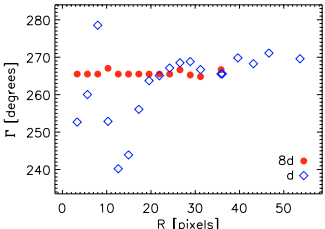

(c) Galaxy 3

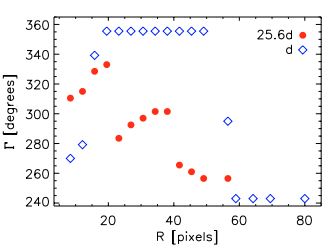

(h) Galaxy 8

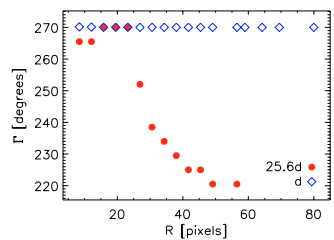

(l) Galaxy 12

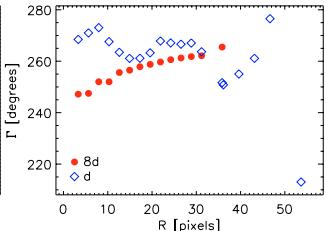

(d) Galaxy 4

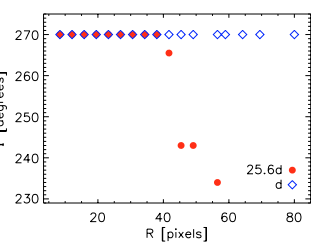

(i) Galaxy 9

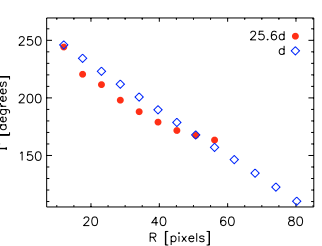

(e) Galaxy 5

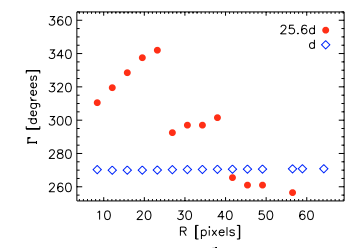

(m) Galaxy 13

Fig. A.5. Kinematic position angle as a function of radius for the original velocity field (blue) together with the velocity field at the largest distance (red). The radius unit is the pixels of the original velocity field.
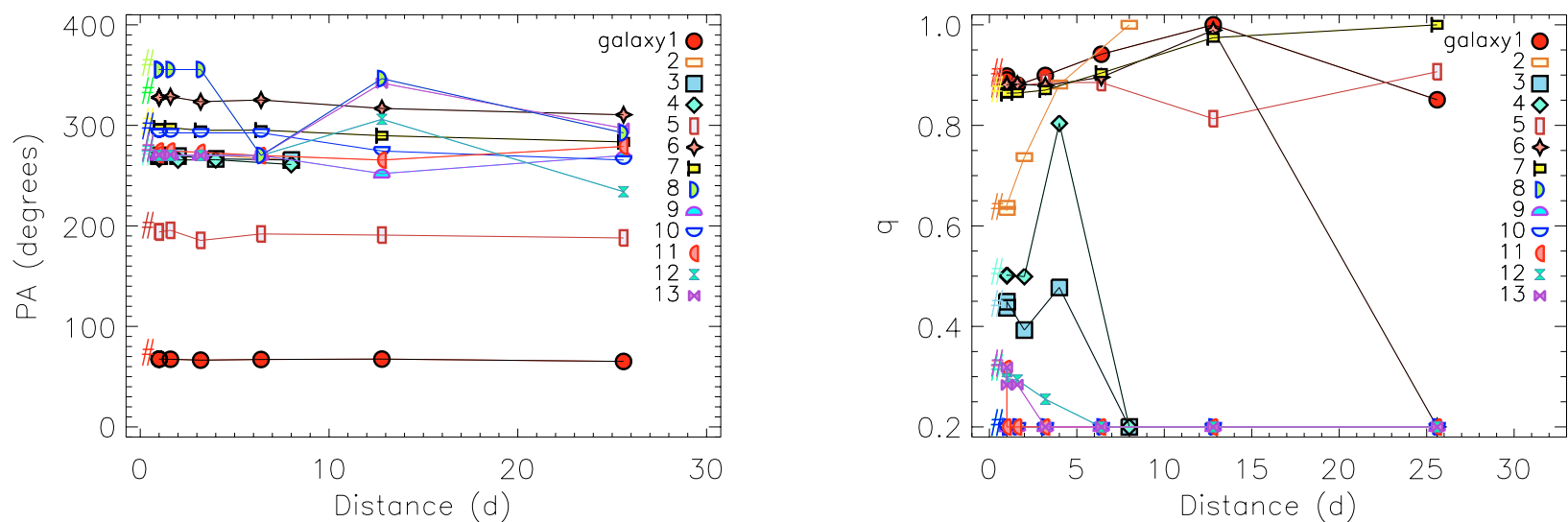

Fig. A.6. Left: the global kinematic position angle as a function of the distance. Right: the global kinematic flattening as a function of the distance. 


\section{Appendix B: Individual galaxies}

Here we give some information on each object in our sample. In the figures, the HST image and the velocity field of each galaxy have the same orientation (the slit position is parallel to the $x$-axis). The velocities and positions are given with respect to the continuum center of the galaxies.

\section{B.1. Cluster galaxies}

Galaxy C1: has very weak OII emission that is not strong enough to provide a velocity field. Stellar and gas rotation curves obtained along the central slit are given in Fig. B.1b.

Galaxy C2: this is a spiral galaxy that has no emission (Fig. B.2).

Galaxy C3: has very weak OII emission that is not strong enough to provide a velocity field (Fig. B.2).

Galaxy C4: this is a galaxy that has no emission. It was observed with only one slit position (Fig. B.2).

Galaxies C5 and C6: they were observed with the same slit (Fig. B.3a). Velocity fields of both are very irregular, which means that they are probably interacting (Fig. B.3e). The stellar and gas rotation curves of galaxy C5 plotted together also show that the gas is disturbed (Fig. B.3b).

Galaxy C5 is an example of the fact that a galaxy with very complex kinematics can have a very regular surface brightness profile. Using its asymmetry and concentration indexes, it is classified as an E/S0 galaxy (Sect. 3.2, Fig. 9).

Galaxy C7: the mean misalignment between its kinematic and photometric axes is large $\left(\Delta \phi=68^{\circ} \pm 26^{\circ}\right)$ and the standard deviation of its kinematic position angle is big $\left(23^{\circ}\right)$. So this galaxy has irregular gas kinematics (Fig. B.4). It is classified as an irregular galaxy according to its photometric asymmetry and concentration measurements (Sect. 3.2, Fig. 9). The stellar and gas velocity fields of this galaxy have some discrepancies.

Galaxy C8: even though it is very close to the cluster center $(0.7 \mathrm{Mpc})$ in projection, this galaxy has regular gas kinematics according to all criteria we defined $\left(\sigma_{\mathrm{PA}}, \Delta \phi\right.$ and $\left.k_{3,5} / k_{1}\right)$ (Fig. B.5).

Galaxy C9: since the signal in one of the slits is not enough to extract a rotation curve, data from just two slits were used to construct its OII velocity field (Fig. B.6e). The average misalignment between its kinematic and photometric axes is very large $\left(\Delta \phi=66^{\circ} \pm 20^{\circ}\right)$. There is also a big discrepancy between its stellar and gas rotation curves. (a)

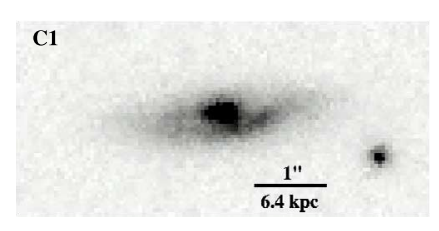

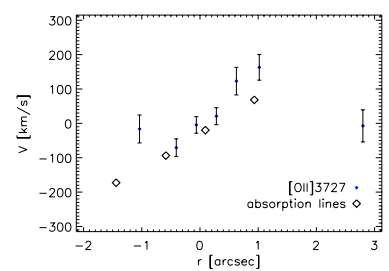

(b)
Fig. B.1. a) HST-ACS image of the galaxy in the $I$ band. b) Rotation curves of the [OII]3727 emission line and several absorption lines extracted along the central slit.
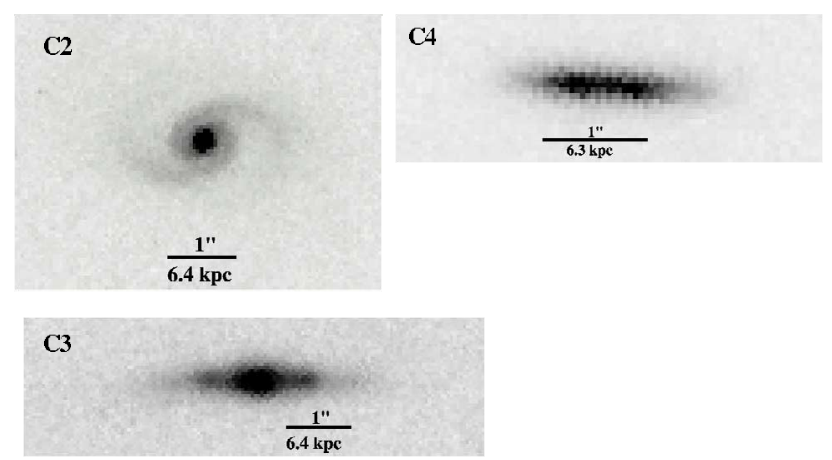

Fig. B.2. I band HST-ACS images of galaxies that do not have emission. The corresponding galaxy name $(\mathrm{C} 2-\mathrm{C} 4)$ is indicated on each image.

Galaxy C10: this galaxy is an example of a case where the rotation curves extracted along the central slit look more regular than the rotation curve extracted along the kinematic major axis (Fig. B.7). It has irregular gas kinematics according to the $k_{3,5} / k_{1}$ criterion.

Galaxy C11: this is a galaxy that was classified as an elliptical in the literature (see Table 1). It was observed together with galaxy F8 within the same slit (Fig. B.15). Their spectra are so close together that they could not be distinguished. 
E. Kutdemir et al.: Internal kinematics of spiral galaxies in distant clusters, Online Material $p 7$

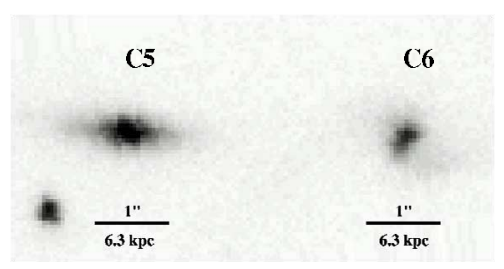

(a)

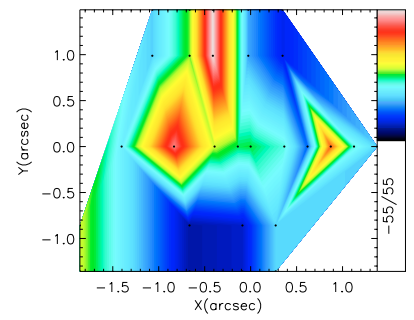

(e.1) [OII] 3727 velocity field of galaxy C5

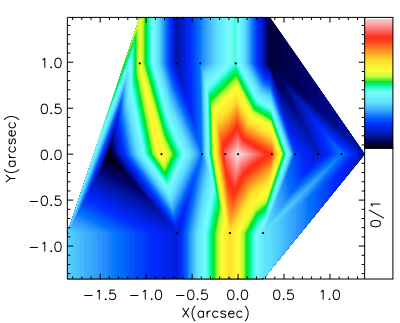

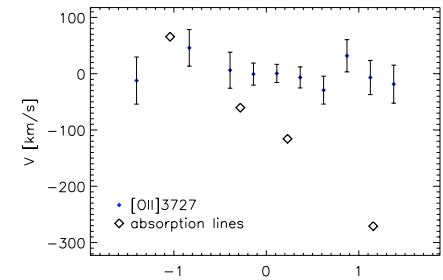

(b.1)

(galaxy C5)

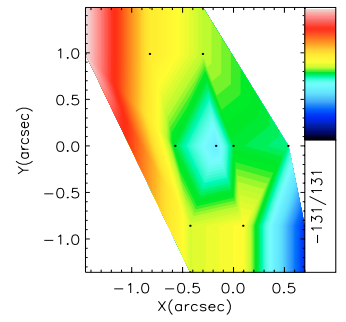

(e.2) [OIII] 3727 velocity field of galaxy $\mathrm{C} 6$

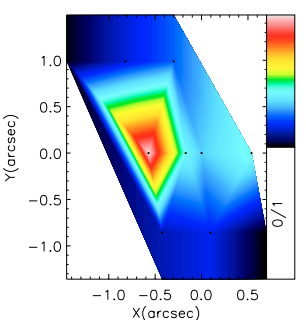

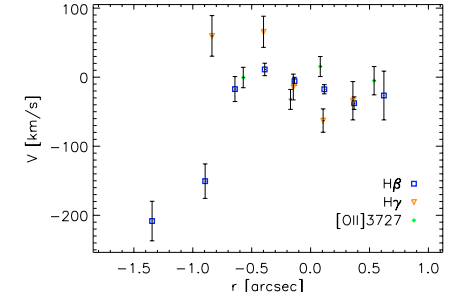

(b.2)

(galaxy C6)

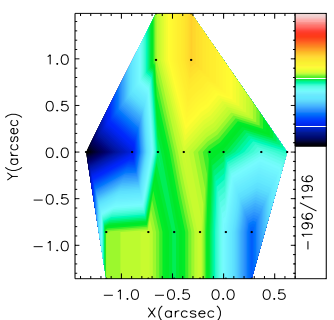

(e.3) $\mathrm{H}_{\beta}$ velocity field of galaxy $\mathrm{C6}$

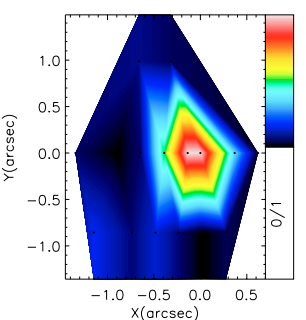

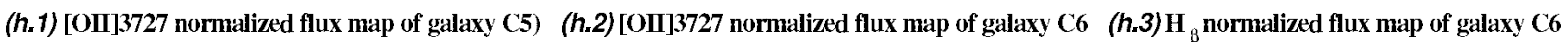

Fig. B.3. a) HST-ACS image of the galaxies in the $I$ band. b.1) Rotation curves of the [OII] emission line and several absorption lines extracted along the central slit for galaxy C5. b.2) Rotation curves of different emission lines extracted along the central slit for galaxy C6. e.1) [OII]3727 velocity field of galaxy C5. e.2) [OII] 3727 velocity field of galaxy C6. e.3) $\mathrm{H} \beta$ velocity field of galaxy C6. h.1) Normalized [OII] 3727 flux map of galaxy C5. h.2) Normalized [OII] 3727 flux map of galaxy C6. h.3) Normalized H $\beta$ flux map of galaxy C6. 
E. Kutdemir et al.: Internal kinematics of spiral galaxies in distant clusters, Online Material $p 8$

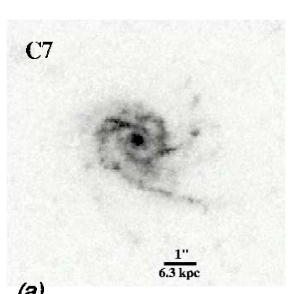

(a)

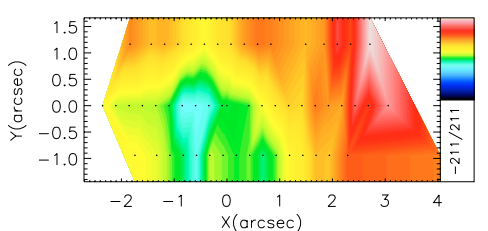

(e)

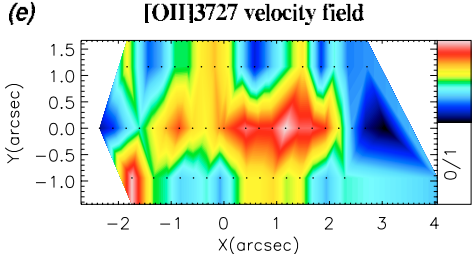

(h.1)

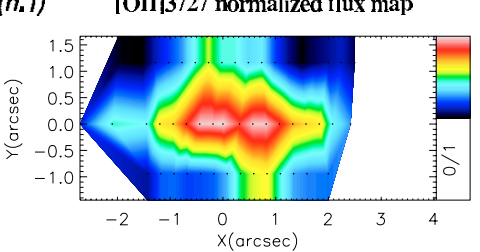

(h.2)

(b)
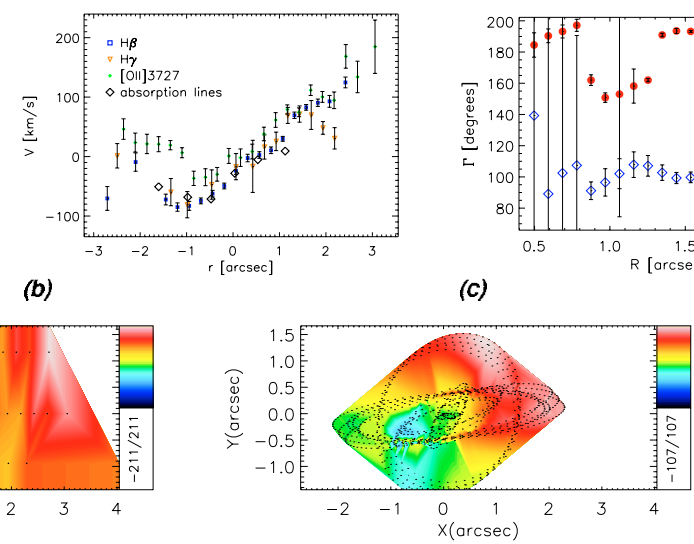

(f)

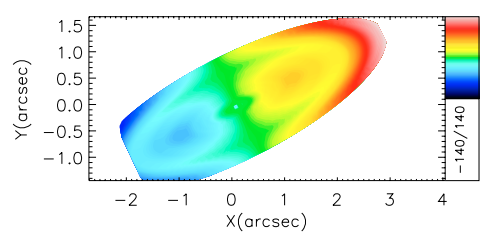

(i)
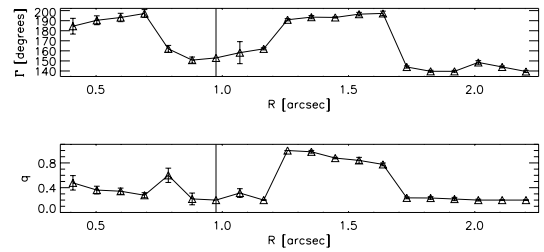

(k)

(c)
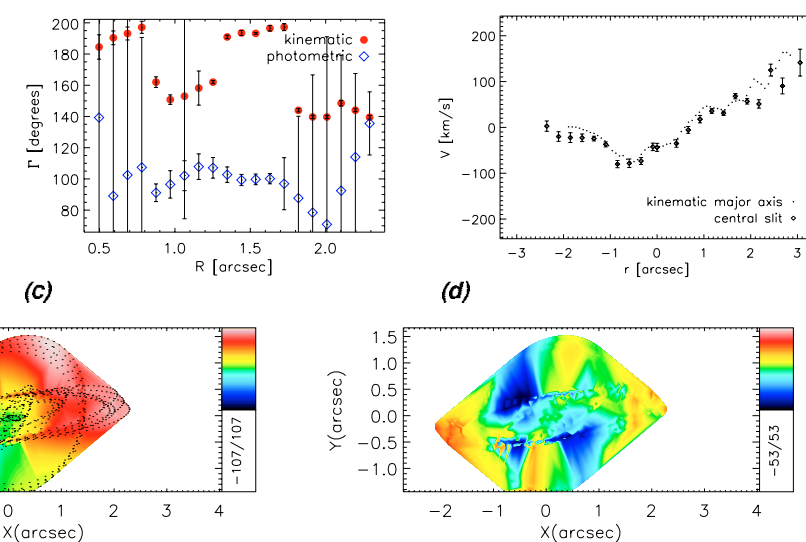

(g)

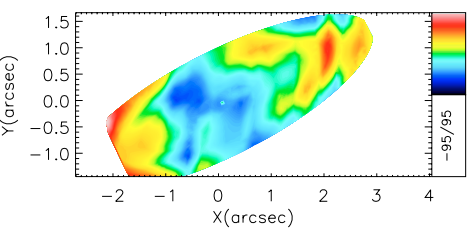

(j)

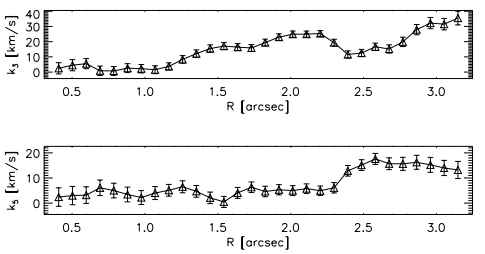

(I)

Fig. B.4. a) HST-ACS image of the galaxy in the $I$ band. b) Rotation curves of different emission lines and several absorption lines extracted along the central slit. c) Position angles of kinematic and photometric axes as a function of radius. d) Rotation curves extracted along the central slit and the kinematic major axis. e) [OII] 3727 velocity field. f) Velocity map reconstructed using 6 harmonic terms. g) Residual of the velocity map and the reconstructed map. h.1) Normalized [OII]3727 flux map. h.2) Normalized H $\beta$ flux map. i) Simple rotation map constructed for position angle and ellipticity fixed to their global values. j) Residual of the velocity map and the simple rotation map. k) Position angle and flattening as a function of radius. I) $k_{3} / k_{1}$ and $k_{5} / k_{1}$ (from the analysis where position angle and ellipticity are fixed to their global values) as a function of radius. 
E. Kutdemir et al.: Internal kinematics of spiral galaxies in distant clusters, Online Material p 9
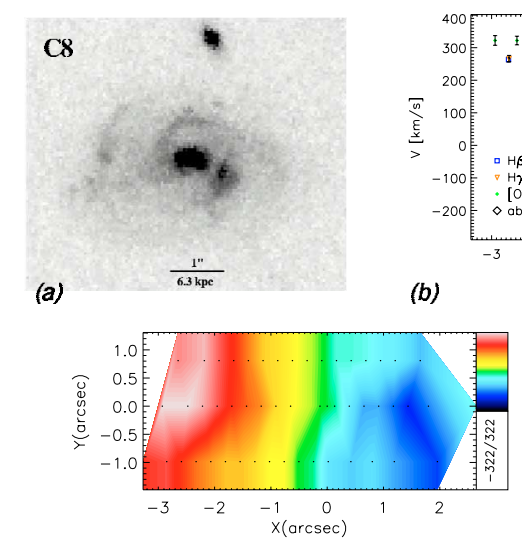

(e)

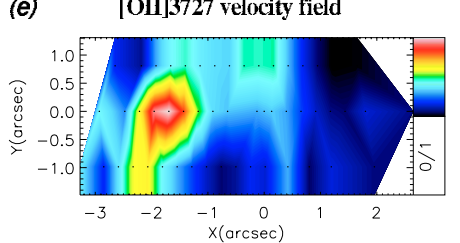

(h.1) [OII] 3727 normalized flux map

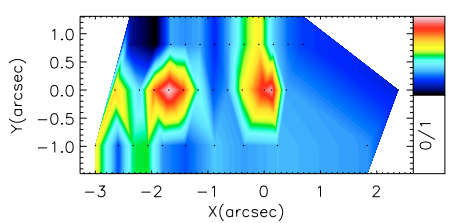

(h.2) $\mathbf{H} \beta$ nornalized flux map

(b)
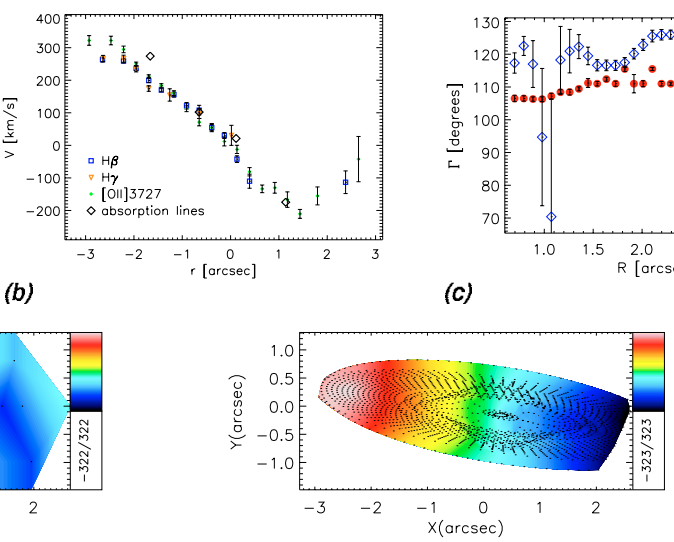

(f) reconstructed velocity map

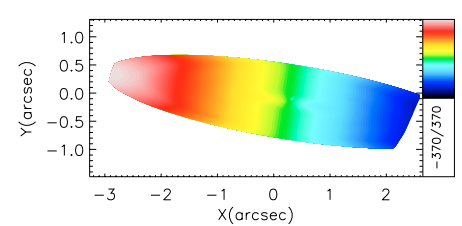

(i) VF for fixed I' and $q$
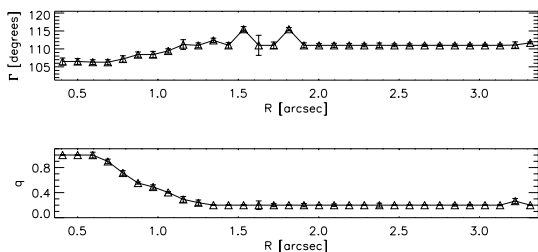

$(k)$

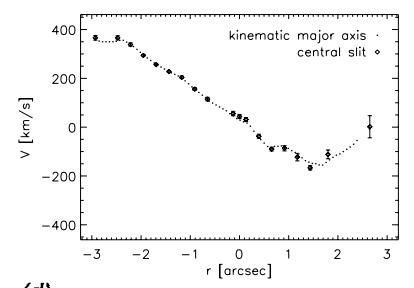

(d)

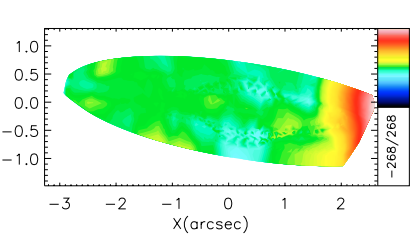

(g)

residual of ' $e$ ' and ' $f$ '

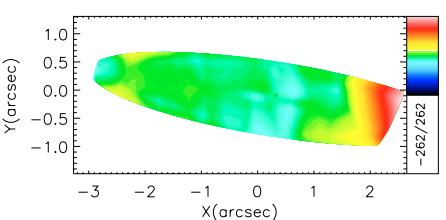

(i) residual of ' $\mathbf{e}$ ' and ' $\mathbf{i}$ '
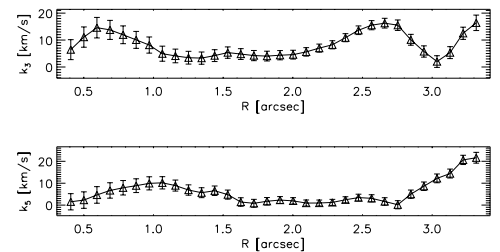

(l)

Fig. B.5. a) HST-ACS image of the galaxy in the $I$ band. b) Rotation curves of different emission lines and several absorption lines extracted along the central slit. c) Position angles of kinematic and photometric axes as a function of radius. d) Rotation curves extracted along the central slit and the kinematic major axis. e) [OII] 3727 velocity field. f) Velocity map reconstructed using 6 harmonic terms. g) Residual of the velocity map and the reconstructed map. h.1) Normalized [OII]3727 flux map. h.2) Normalized H $\beta$ flux map. i) Simple rotation map constructed for position angle and ellipticity fixed to their global values. $\mathbf{j}$ ) Residual of the velocity map and the simple rotation map. k) Position angle and flattening as a function of radius. l) $k_{3} / k_{1}$ and $k_{5} / k_{1}$ (from the analysis where position angle and ellipticity are fixed to their global values) as a function of radius. 
E. Kutdemir et al.: Internal kinematics of spiral galaxies in distant clusters, Online Material p 10

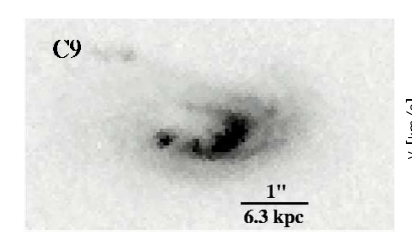

(a)

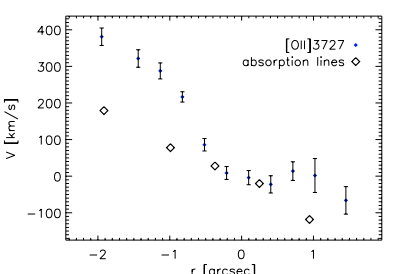

(b)

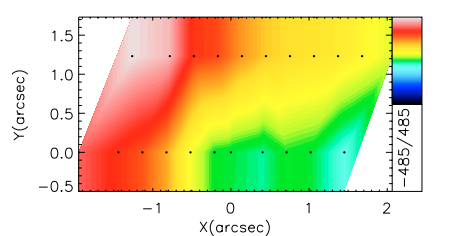

(e)

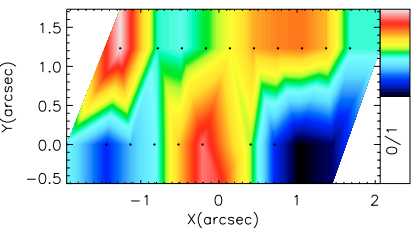

(h)

[OII] 3727 normalized flux map
(1)
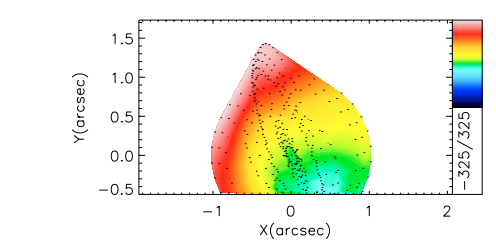

(f) reconstructed velocity map

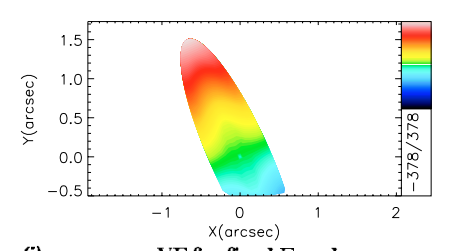

(i) VF for fixed $\Gamma$ and $q$
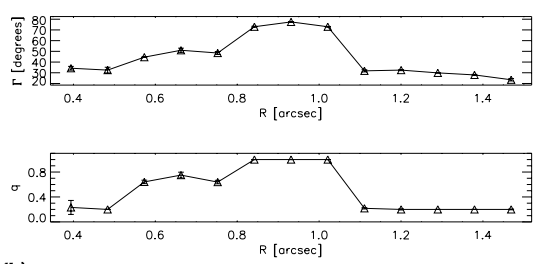

(k)

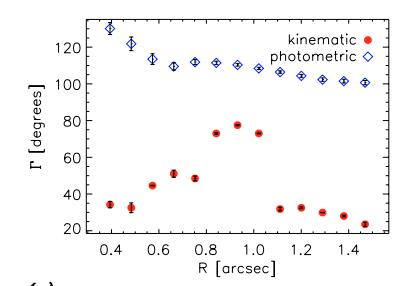

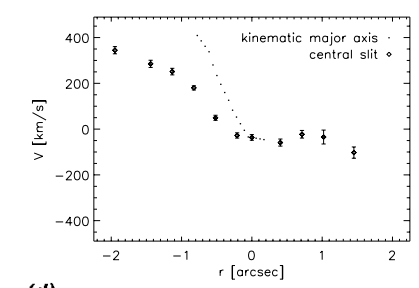

(d)

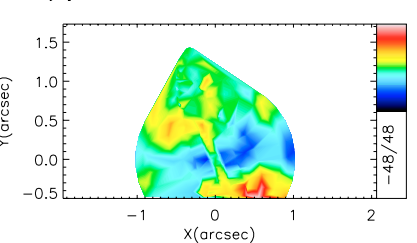

(g)
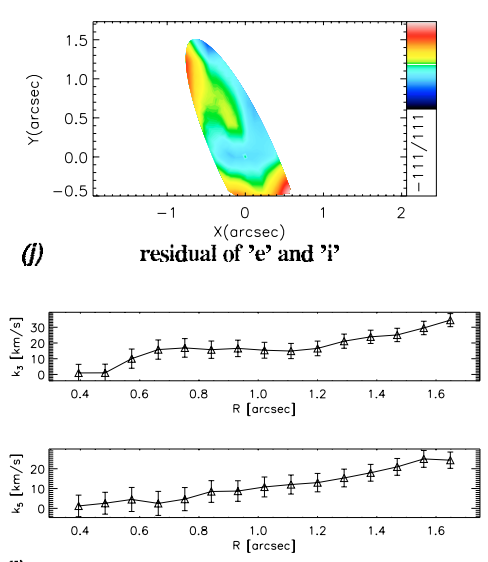

(l)

Fig. B.6. a) HST-ACS image of the galaxy in the $I$ band. b) Rotation curves of OII emission line and several absorption lines extracted along the central slit. c) Position angles of kinematic and photometric axes as a function of radius. d) Rotation curves extracted along the central slit and the kinematic major axis. e) [OII] 3727 velocity field. f) Velocity map reconstructed using 6 harmonic terms. g) Residual of the velocity map and the reconstructed map. h) Normalized [OII] 3727 flux map. i) Simple rotation map constructed for position angle and ellipticity fixed to their global values. j) Residual of the velocity map and the simple rotation map. k) Position angle and flattening as a function of radius. I) $k_{3} / k_{1}$ and $k_{5} / k_{1}$ (from the analysis where position angle and ellipticity are fixed to their global values) as a function of radius. 
E. Kutdemir et al.: Internal kinematics of spiral galaxies in distant clusters, Online Material p 11

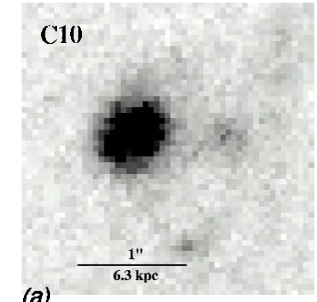

(a)

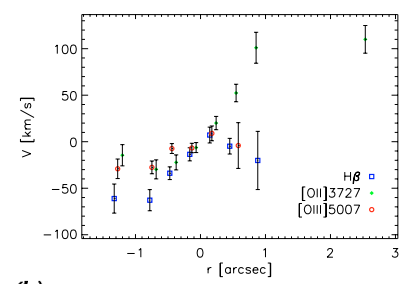

(b)

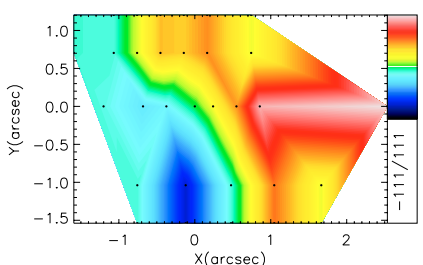

(e) $[0 \Pi 3727$ velocity field

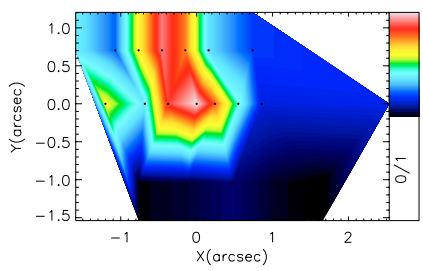

(h) [OII] 3727 normalized flux map

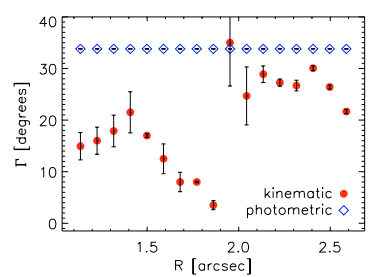

(c)

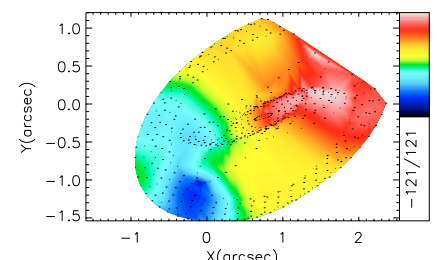

(f)

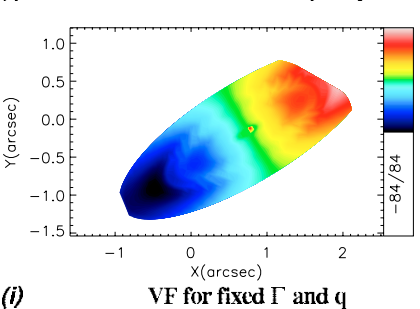

(i)

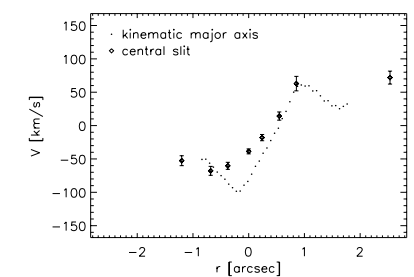

(d)

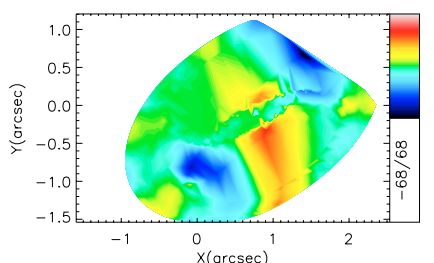

(g)

residual of 'es' and 'p

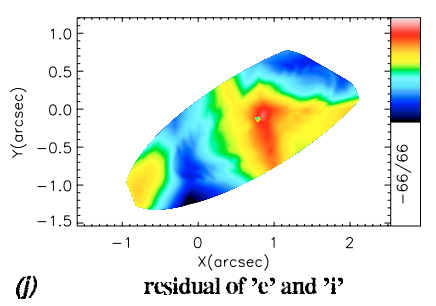

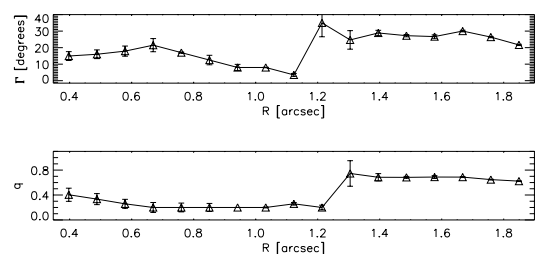

(k)

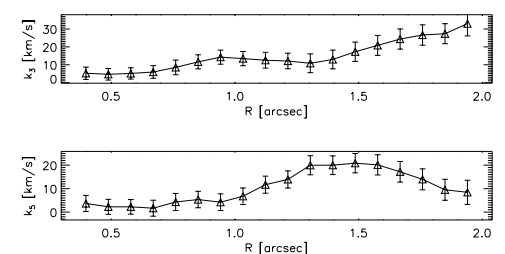

(I)

Fig. B.7. a) HST-ACS image of the galaxy in the $I$ band. b) Rotation curves of different emission lines extracted along the central slit. c) Position angles of kinematic and photometric axes as a function of radius. d) Rotation curves extracted along the central slit and the kinematic major axis. e) [OII] 3727 velocity field. f) Velocity map reconstructed using 6 harmonic terms. g) Residual of the velocity map and the reconstructed map. h) Normalized [OII]3727 flux map. i) Simple rotation map constructed for position angle and ellipticity fixed to their global values. j) Residual of the velocity map and the simple rotation map. k) Position angle and flattening as a function of radius. I) $k_{3} / k_{1}$ and $k_{5} / k_{1}$ (from the analysis where position angle and ellipticity are fixed to their global values) as a function of radius. 


\section{B.2. Field galaxies}

Galaxy F1: it is a galaxy at $z=0.9009$ that was observed using only one slit position (Fig. B.8).

Galaxy F2: background galaxy at $z=0.5795$ (Fig. B.9). It has regular gas kinematics according to the $\sigma_{\mathrm{PA}}$ and $k_{3,5} / k_{1}$ criteria. Since it is nearly face-on, its $\Delta \phi$ was excluded from the analysis.

Galaxy F3: background galaxy at $z=0.5667$ (Fig. B.10). It has irregular gas kinematics according to the $\Delta \phi$ criterion $\left(\Delta \phi=39^{\circ} \pm 9^{\circ}\right)$. Classified as an irregular galaxy according to its photometric asymmetry and concentration measurements (Sect. 3.2, Fig. 9).

Galaxy F4: foreground galaxy at $z=0.1867$ (Fig. B.11). It has irregular gas kinematics according to the $\sigma_{\mathrm{PA}}$ and $k_{3,5} / k_{1}$ criteria. After $R \sim 1^{\prime \prime} .3$, there is a substantial change in its kinematic position angle and ellipticity which may be the indicator of a secondary component.

Galaxy F5: foreground galaxy at $z=0.1573$. The spectral range of our observations cover several emission lines at this redshift. Rotation curves extracted along the central slit using these lines are plotted together in Fig. A.12b. It has irregular kinematics according to the $\Delta \phi$ criterion. The $k_{3,5} / k_{1}$ is not meaningful since the measurements cover only the inner part of the velocity field in this particular case (see Fig. A.12i). The reason is that $k_{3,5} / k_{1}$ is calculated while fixing the position angle to its global value and the misalignment between this angle and the slit position angle is very large. Therefore $k_{3,5} / k_{1}$ of this galaxy is excluded from our analysis.

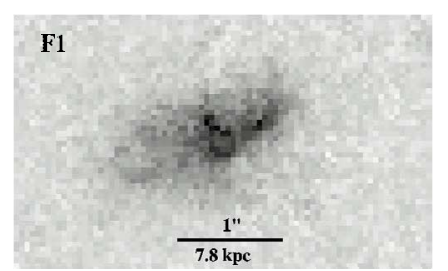

(a)

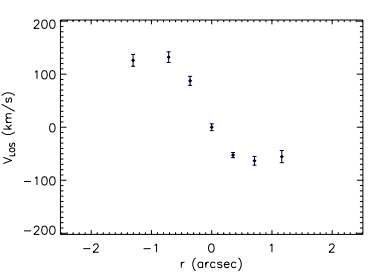

(b)
Fig. B.8. a) HST-ACS image of the galaxy in the $I$ band. b) [OII] 3727 rotation curve (this galaxy was observed with only one slit position).

Galaxy F6: foreground galaxy at $z=0.0982$. As the sudden change in its kinematic position angle indicates (Fig. B.13k), and can be seen in its $\mathrm{H} \alpha$ velocity field (Fig. B.13e), it has a counter rotating core. Related to this is the fact that it has irregular gas kinematics according to all the criteria we defined: $\Delta \phi=57^{\circ} \pm$ $44^{\circ}, \sigma_{\mathrm{PA}}=29^{\circ}, k_{3,5} / k_{1}=0.27 \pm 0.18$.

Galaxy F7: only one emission line is visible in its spectrum and no other feature can be identified. Therefore we could not measure its redshift and used the value given in the literature for our analysis $(z=0.9125$, Moran 2008). It has regular kinematics according to all the criteria we defined $\left(\sigma_{\mathrm{PA}}, k_{3,5} / k_{1}\right.$ and $\left.\Delta \phi\right)$ (Fig. B.14). Using its photometry (asymmetry and concentration indexes), it is classified as an irregular/peculiar galaxy (Fig. 9). 
E. Kutdemir et al.: Internal kinematics of spiral galaxies in distant clusters, Online Material p 13
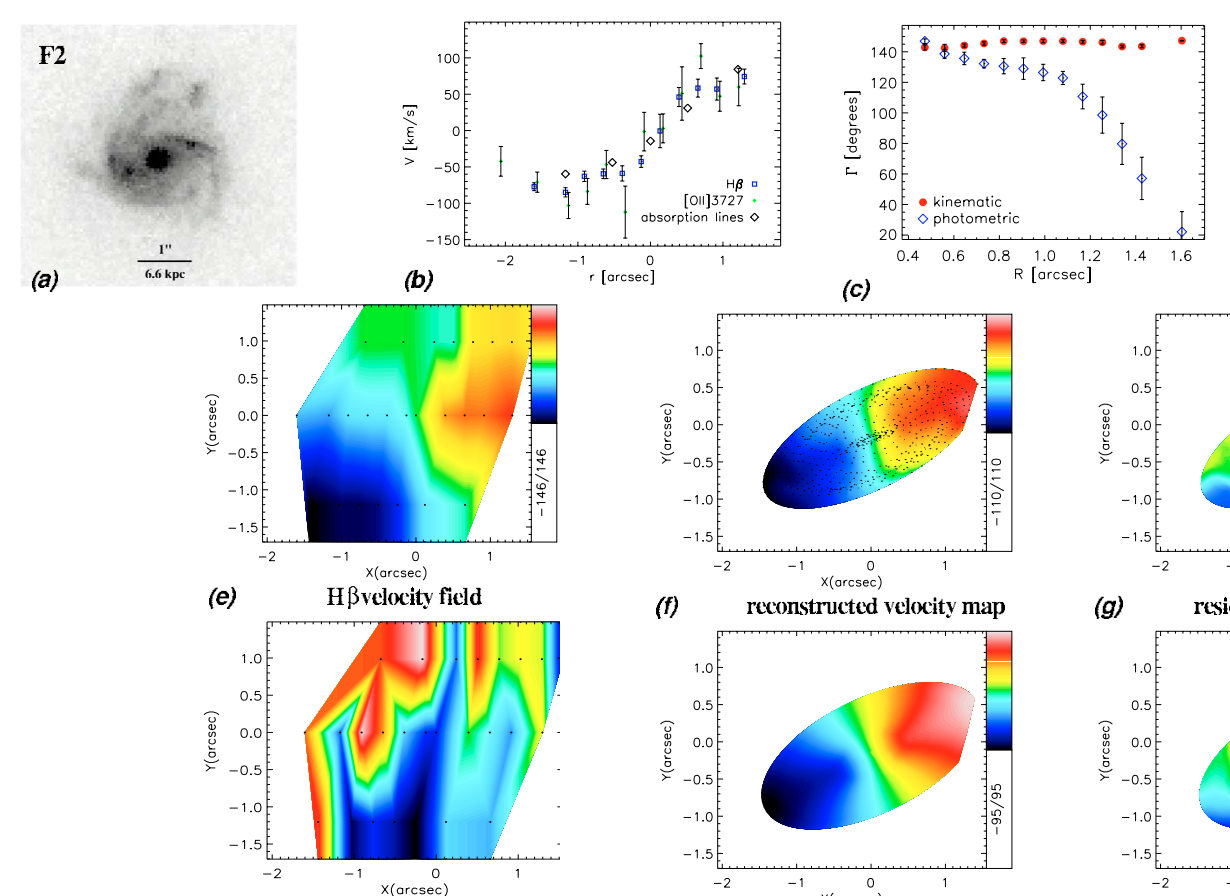

(f)
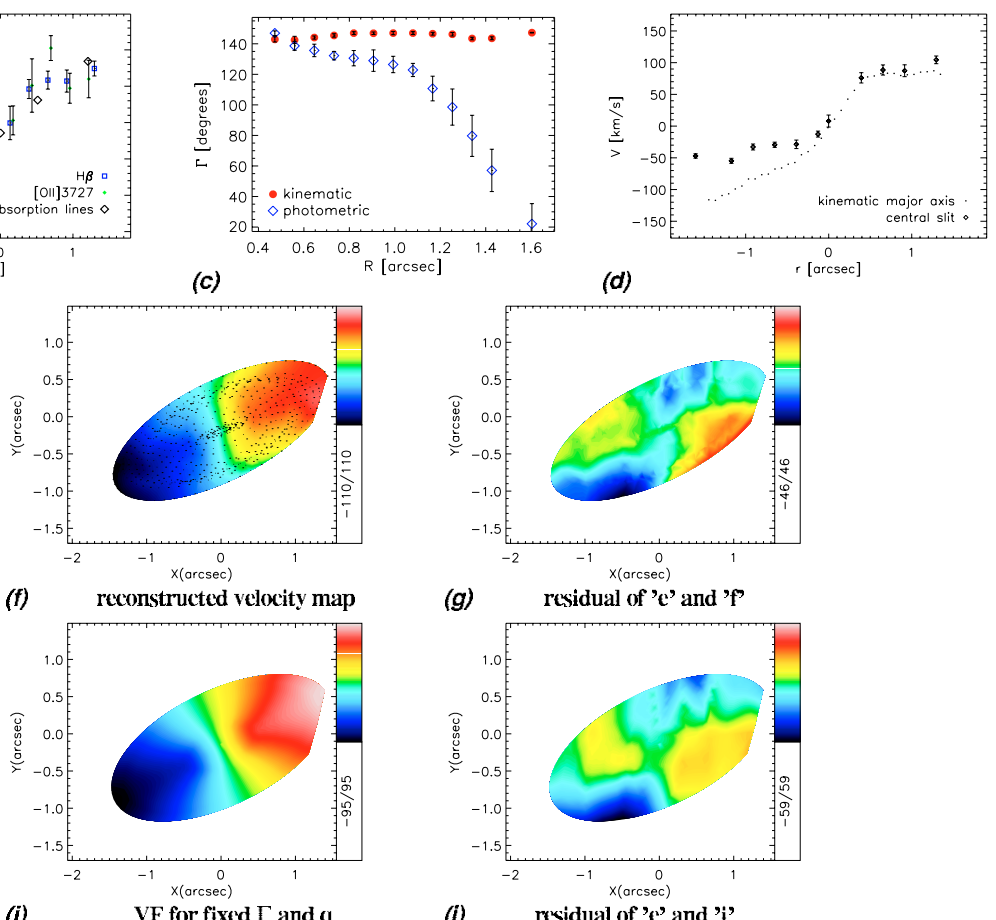

(h)

$\mathrm{H} \beta$ normalized flux map

(i)

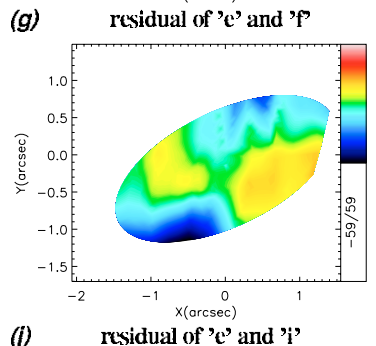

residual of ' $\mathrm{e}$ ' and ' $\mathrm{f}$

residual of 'c' and ' $i$ '

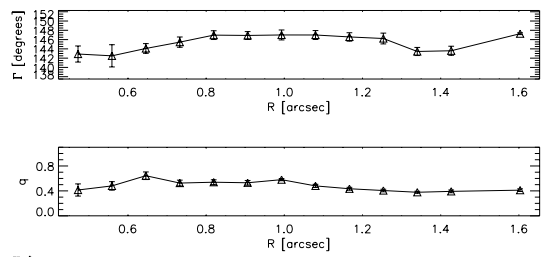

(k)

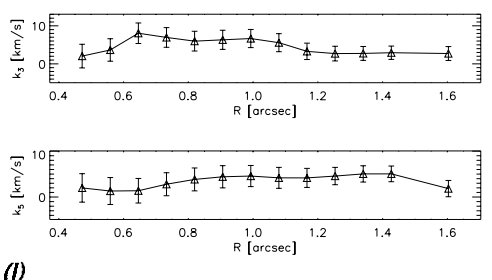

Fig. B.9. a) HST-ACS image of the galaxy in the $I$ band. b) Rotation curves of different emission lines and several absorption lines extracted along the central slit. c) Position angles of kinematic and photometric axes as a function of radius. d) Rotation curves extracted along the central slit and the kinematic major axis. e) $\mathrm{H} \beta$ velocity field. f) Velocity map reconstructed using 6 harmonic terms. g) Residual of the velocity map and the reconstructed map. h) Normalized $\mathrm{H} \beta$ flux map. i) Simple rotation map constructed for position angle and ellipticity fixed to their global values. j) Residual of the velocity map and the simple rotation map. k) Position angle and flattening as a function of radius. I) $k_{3} / k_{1}$ and $k_{5} / k_{1}$ (from the analysis where position angle and ellipticity are fixed to their global values) as a function of radius. 
E. Kutdemir et al.: Internal kinematics of spiral galaxies in distant clusters, Online Material p 14
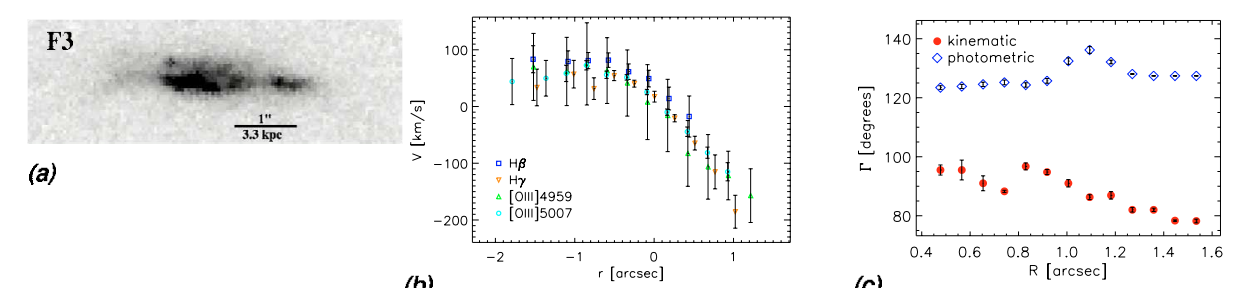

(c)

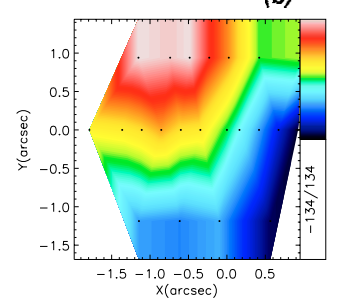

(e) [OIII 5007 velocity ficd

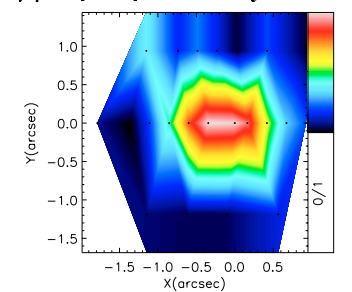

(h) [OHII] 5007 normalized flux map

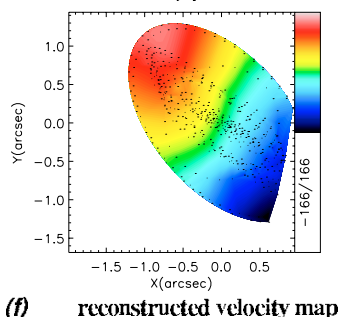

(f)

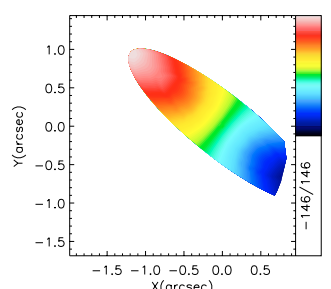

(i) $\quad V F$ for fixed $\Gamma$ and $q$

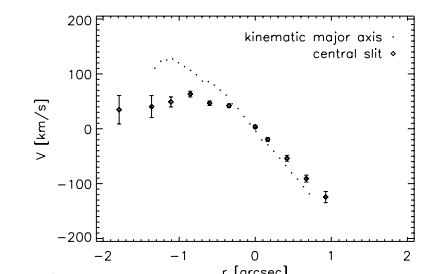

(d)

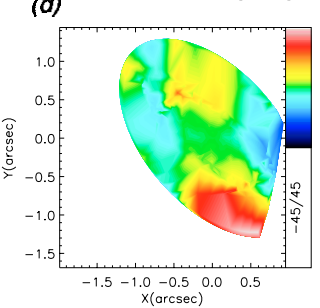

(g)

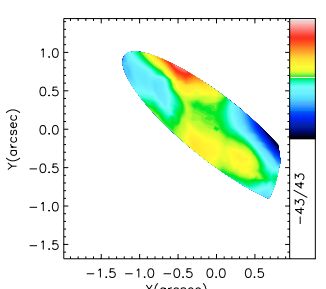

(j) residual of ' $'$ ' and ' $\mathrm{i}$ '
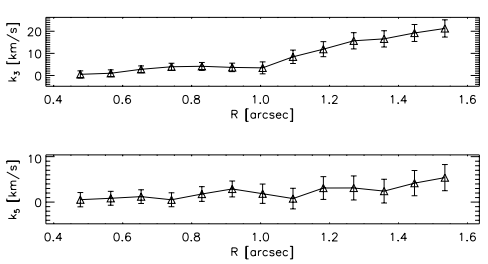

(I)

Fig. B.10. a) HST-ACS image of the galaxy in the $I$ band. b) Rotation curves of different emission lines extracted along the central slit. c) Position angles of kinematic and photometric axes as a function of radius. d) Rotation curves extracted along the central slit and the kinematic major axis. e) [OIII]5007 velocity field. f) Velocity map reconstructed using 6 harmonic terms. g) Residual of the velocity map and the reconstructed map. h) Normalized [OII]3727 flux map. i) Simple rotation map constructed for position angle and ellipticity fixed to their global values. j) Residual of the velocity map and the simple rotation map. k) Position angle and flattening as a function of radius. I) $k_{3} / k_{1}$ and $k_{5} / k_{1}$ (from the analysis where position angle and ellipticity are fixed to their global values) as a function of radius. 
E. Kutdemir et al.: Internal kinematics of spiral galaxies in distant clusters, Online Material p 15

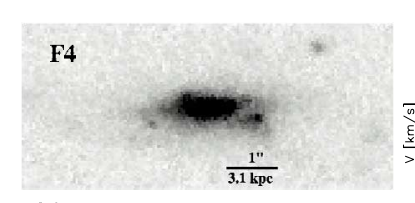

(a)

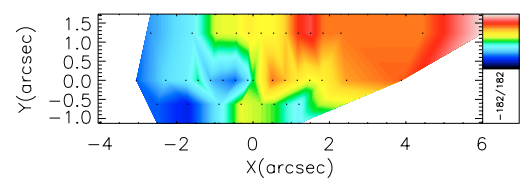

(e)

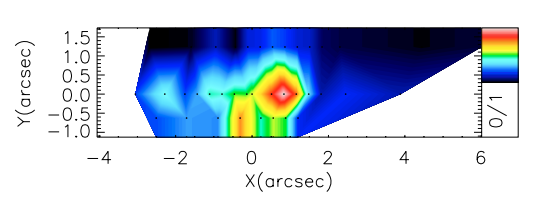

(h)

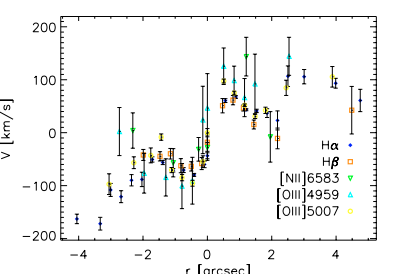

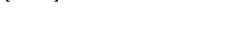

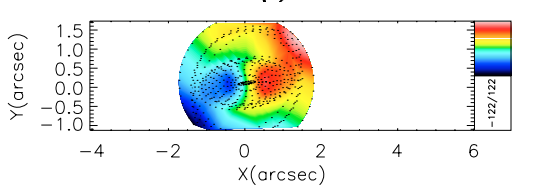

$(f)$

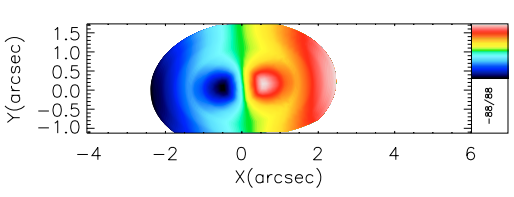

(i)

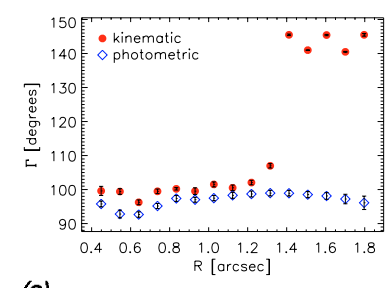

$(d)$

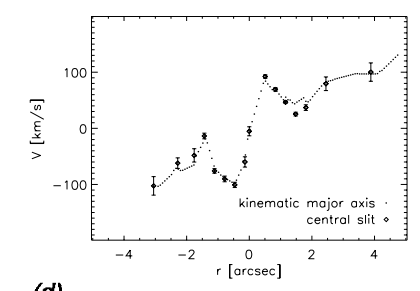

(d)

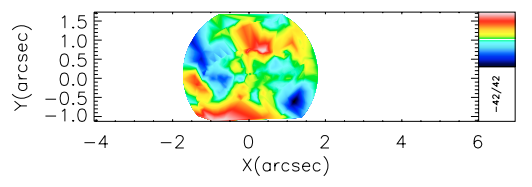

(g)

residual of ' $e$ ' and ' $f$ '

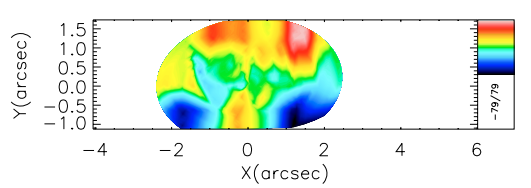

(j)

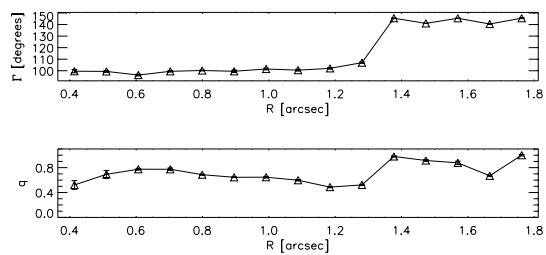

(k)

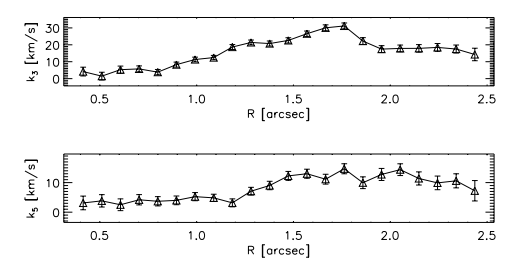

(I)

Fig. B.11. a) HST-ACS image of the galaxy in the $I$ band. b) Rotation curves of different emission lines extracted along the central slit. c) Position angles of kinematic and photometric axes as a function of radius. d) Rotation curves extracted along the central slit and the kinematic major axis. e) [OIII]5007 velocity field. f) Velocity map reconstructed using 6 harmonic terms. g) Residual of the velocity map and the reconstructed map. h) Normalized [OIII]5007 flux map. i) Simple rotation map constructed for position angle and ellipticity fixed to their global values. j) Residual of the velocity map and the simple rotation map. k) Position angle and flattening as a function of radius. I) $k_{3} / k_{1}$ and $k_{5} / k_{1}$ (from the analysis where position angle and ellipticity are fixed to their global values) as a function of radius. 
E. Kutdemir et al.: Internal kinematics of spiral galaxies in distant clusters, Online Material p 16

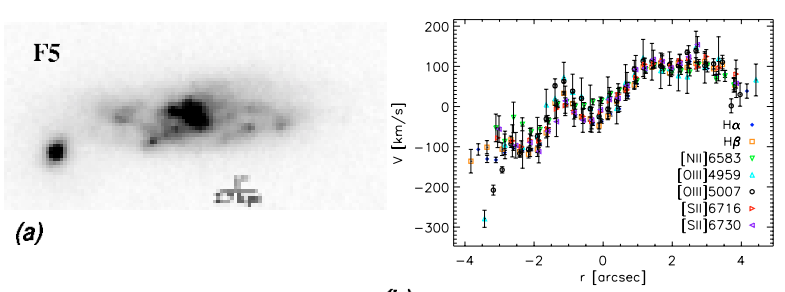

(b)

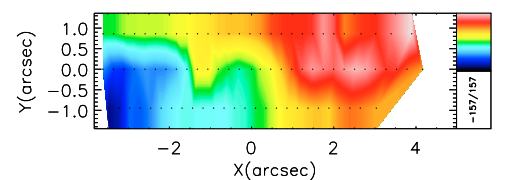

(e)

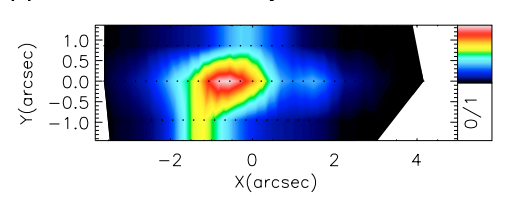

(h)

H $\propto$ normalized flux map

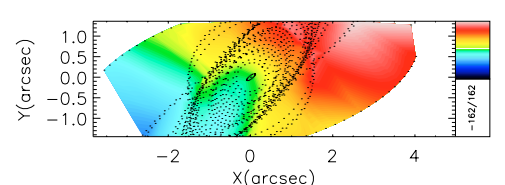

(f)

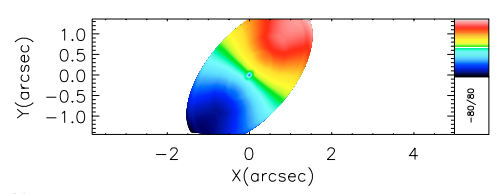

(i) VF for fixed I' and $q$
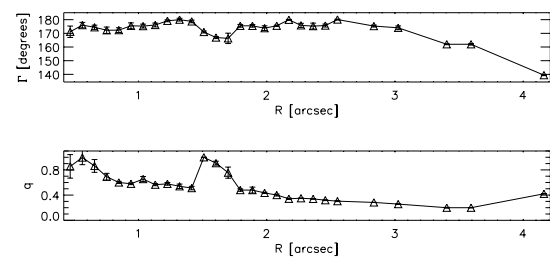

(k)

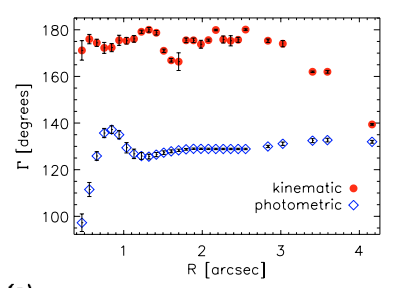

(d)

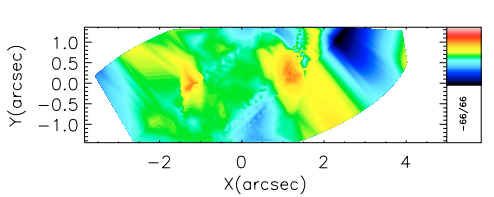

(g)

residual of ' $t$ ' and ' $f$ '

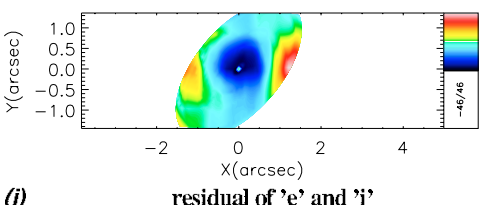

(i)

residual of ' $\mathrm{e}$ ' and ' $\mathrm{i}$ '
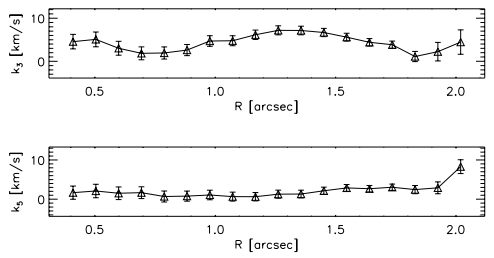

(I)

Fig. B.12. a) HST-ACS image of the galaxy in the $I$ band. b) Rotation curves of different emission lines extracted along the central slit. c) Position angles of kinematic and photometric axes as a function of radius. d) Rotation curves extracted along the central slit and the kinematic major axis. e) $\mathrm{H} \alpha$ velocity field. f) Velocity map reconstructed using 6 harmonic terms. g) Residual of the velocity map and the reconstructed map. h) Normalized $\mathrm{H} \alpha$ flux map. i) Simple rotation map constructed for position angle and ellipticity fixed to their global values. j) Residual of the velocity map and the simple rotation map. k) Position angle and flattening as a function of radius. l) $k_{3} / k_{1}$ and $k_{5} / k_{1}$ (from the analysis where position angle and ellipticity are fixed to their global values) as a function of radius. 
E. Kutdemir et al.: Internal kinematics of spiral galaxies in distant clusters, Online Material p 17

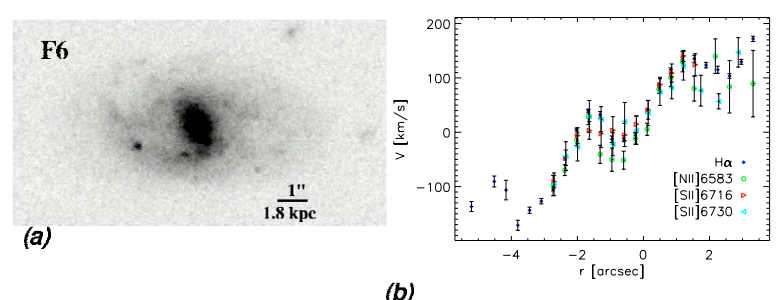

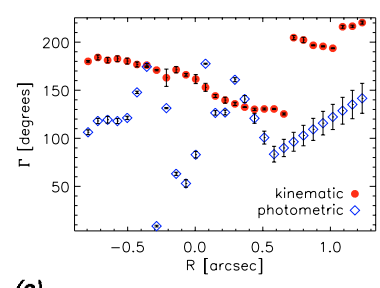

(c)

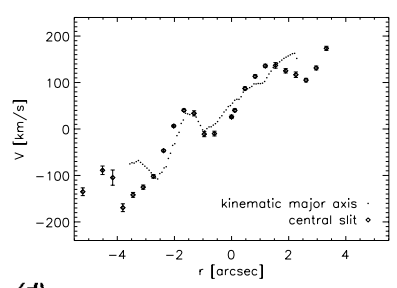

(d)

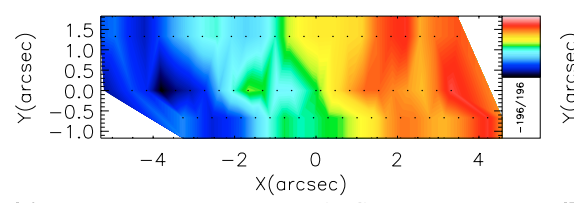

(e)

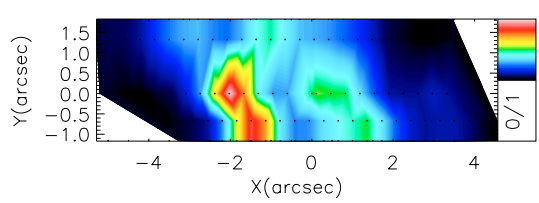

(h.t) (f)

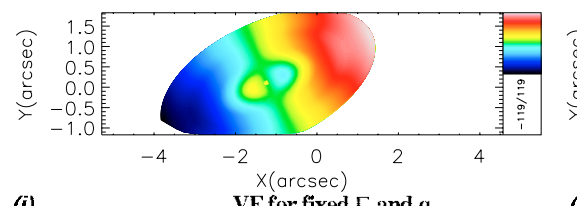

(i)

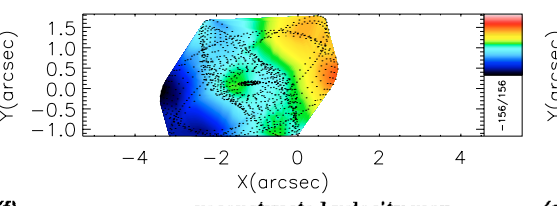

reconstructed velocity map

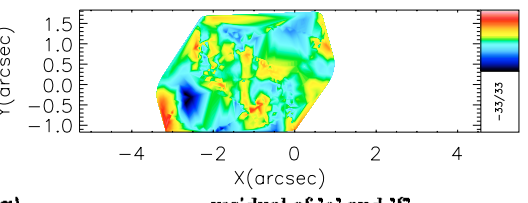

(g) residual of ' $e$ ' and 'f

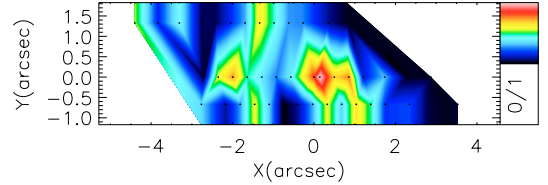

(h.2)

[SII]6730 normalized flux map

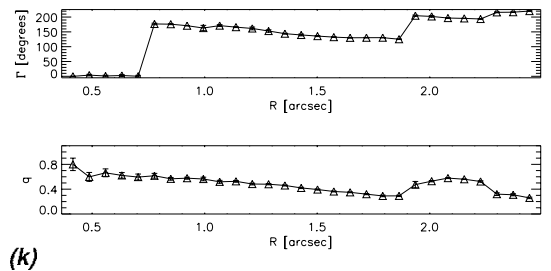

(j)

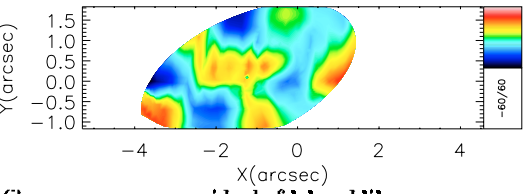

residual of ' $\mathrm{e}$ ' and ' $\mathrm{i}$ '

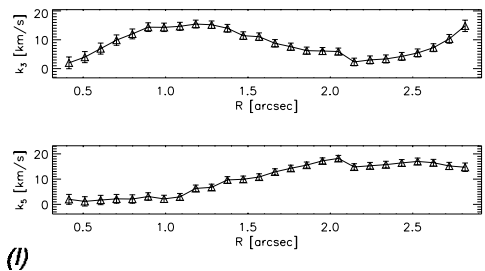

(I)

Fig. B.13. a) HST-ACS image of the galaxy in the $I$ band. b) Rotation curves of different emission lines extracted along the central slit. c) Position angles of kinematic and photometric axes as a function of radius. d) Rotation curves extracted along the central slit and the kinematic major axis. e) $\mathrm{H} \alpha$ velocity field. f) Velocity map reconstructed using 6 harmonic terms. g) Residual of the velocity map and the reconstructed map. h.1) Normalized $\mathrm{H} \alpha$ flux map. h.2) Normalized S[II]6730 flux map. i) Simple rotation map constructed for position angle and ellipticity fixed to their global values. j) Residual of the velocity map and the simple rotation map. k) Position angle and flattening as a function of radius. I) $k_{3} / k_{1}$ and $k_{5} / k_{1}$ (from the analysis where position angle and ellipticity are fixed to their global values) as a function of radius. 
E. Kutdemir et al.: Internal kinematics of spiral galaxies in distant clusters, Online Material p 18
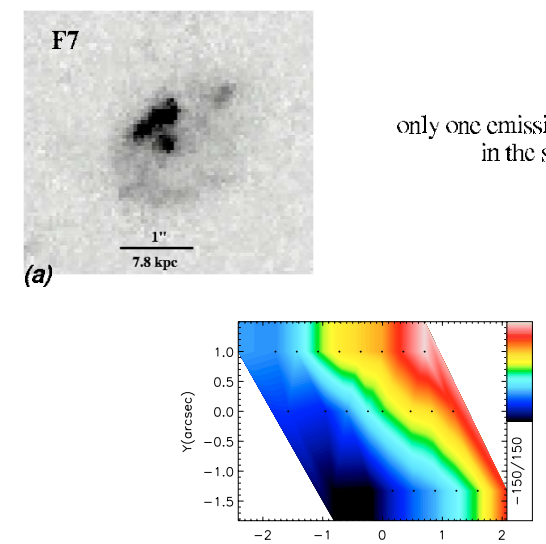

(e)

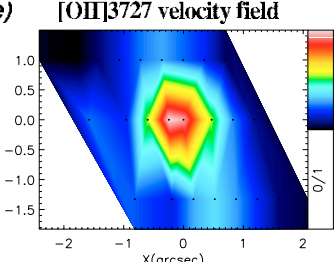

(h) [OIП 3727 normalized flux map

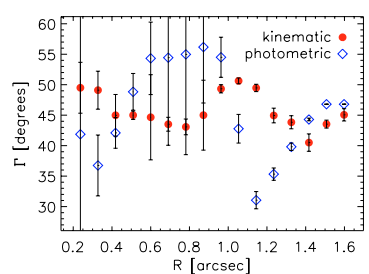

(c)

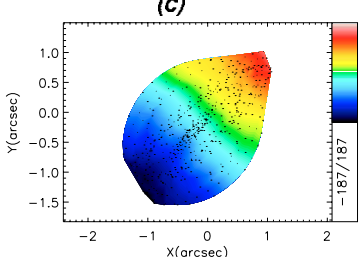

(f) reconstructed velocity map

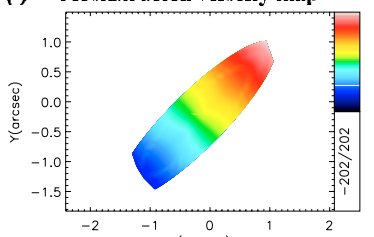

(i) VF for fixed 1 and $q$

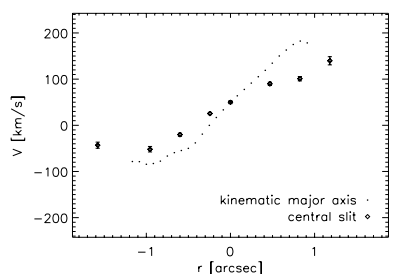

(d)

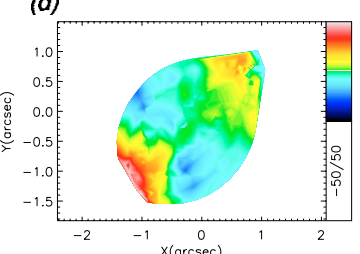

(g)

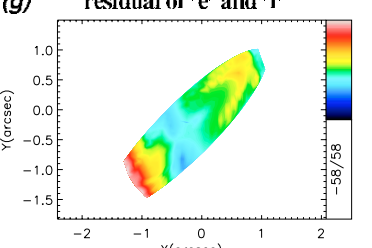

(i) residual of 'e' and ' $\mathrm{i}$ '
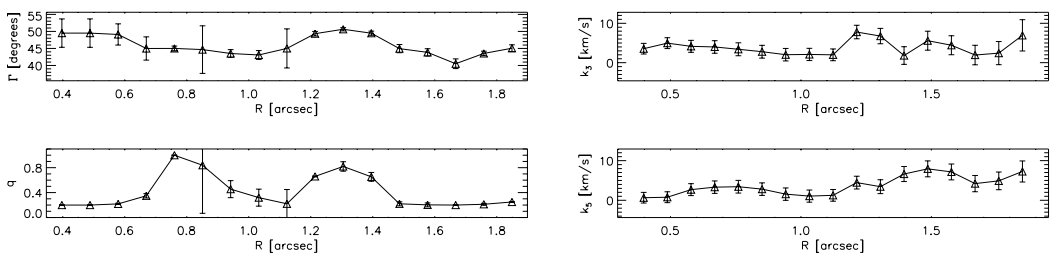

(k)

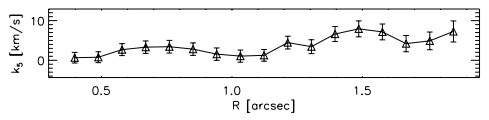

(I)

Fig. B.14. a) HST-ACS image of the galaxy in the $I$ band. c) Position angles of kinematic and photometric axes as a function of radius. d) Rotation curves extracted along the central slit and the kinematic major axis. e) [OII] 3727 velocity field. f) Velocity map reconstructed using 6 harmonic terms. g) Residual of the velocity map and the reconstructed map. h) Normalized [OII]3727 flux map. i) Simple rotation map constructed for position angle and ellipticity fixed to their global values. j) Residual of the velocity map and the simple rotation map. k) Position angle and flattening as a function of radius. l) $k_{3} / k_{1}$ and $k_{5} / k_{1}$ (from the analysis where position angle and ellipticity are fixed to their global values) as a function of radius. 


\section{B.3. Galaxies difficult to treat}

Here we give information on the galaxies that were excluded from the analysis because of the problems explained below for individual cases. This section also includes the galaxies for which the redshift is uncertain, since only one line is visible in the spectrum. In these cases, different possibilities for identification of the line rule out that these galaxies are cluster members. Galaxy F8: this object is so close together to galaxy C11 that on the FORS-spectra, they can not be distinguished (Fig. B.15). The redshift calculated using the emission lines in the composite spectrum is $z=0.4443$. Using the information from the literature (Ellingson et al. 1998) we confirmed that the redshift we measure belongs to galaxy F8. Since the spectra can not be separated, the velocity fields are very noisy and not reliable. Therefore we excluded this object from our analysis.

Galaxy F9: two objects were observed within the same slit with only one slit position (Fig. B.16). A part of the spectrum of galaxy F9 is outside the frame. It is at $z=0.3259$ and has no emission. This galaxy is classified as an E/S0 using its asymmetry-concentration parameters (Sect. 3.2, Fig. 9). It has an exponential disk component according to our surface photometry results (Table C.2), so it could be classified as an S0 galaxy. The other spectrum is so weak, that no line can be identified in the noise.
Galaxy F10: foreground galaxy at $z=0.4947$. It has a tidal tail which indicates a disturbance (Fig. B.17). The [OII]3727 emission line in its spectrum could not be used since it is very noisy with the contribution from a strong sky line. Its velocity field constructed using the second prominent line ([OIII]5007) is quite noisy as well, so this galaxy is excluded from our analysis.

Galaxy F11: only one emission line is visible in its spectrum, and no other feature could be identified. Therefore its redshift is not certain. Its photometric redshift is available in the literature (see Table 1), but we did not use it in our analysis. Since the signal in one of the slits is not enough to extract a rotation curve, data from the two other slits were used to construct its velocity field (Fig. B.18). It has regular kinematics according to all the criteria we defined $\left(\sigma_{\mathrm{PA}}, k_{3,5} / k_{1}\right.$ and $\left.\Delta \phi\right)$. 
E. Kutdemir et al.: Internal kinematics of spiral galaxies in distant clusters, Online Material p 20

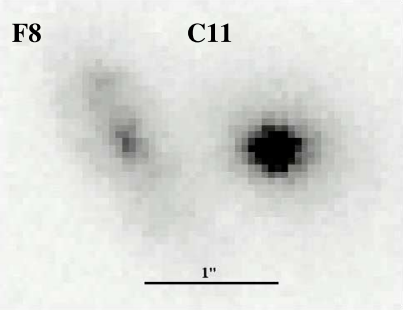

(a)

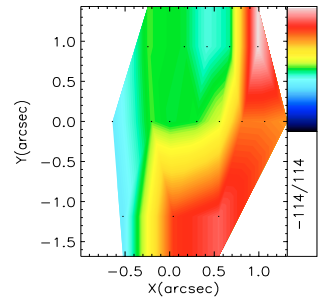

(e.1) $[\mathrm{OII}] 3727$ velocity field

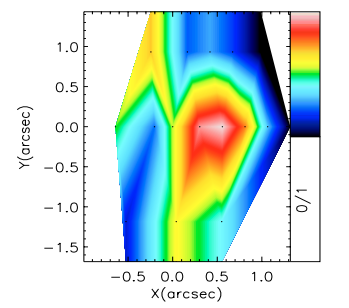

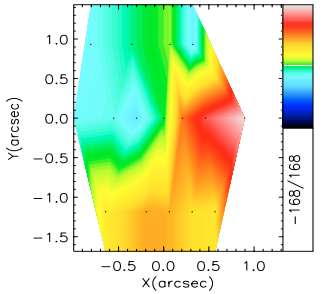

(e.2) $\mathbf{H} \beta$ velocity field

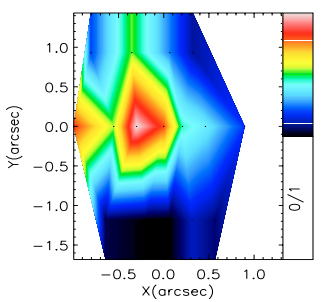

(h.2) $\mathrm{H} \beta$ normalized flux map

Fig. B.15. a) HST-ACS image of the galaxies in the $I$ band. e.1) $[\mathrm{OII}] 3727$ velocity field constructed using composite spectra of the two objects. e.2) $\mathrm{H} \beta$ velocity field constructed using composite spectra of the two objects. h.1) Normalized [OII]3727 flux map. h.2) Normalized $\mathrm{H} \beta$ flux map.

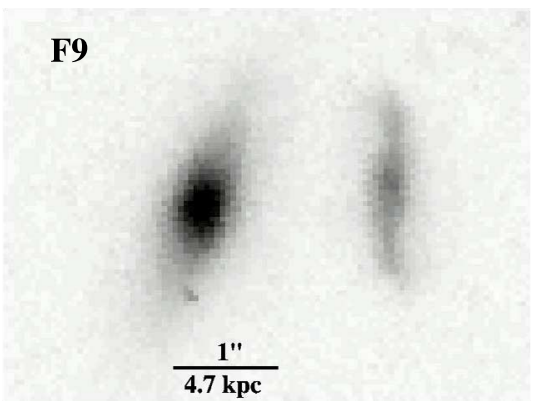

Fig. B.16. HST-ACS image of the galaxies in the $I$ band. 
E. Kutdemir et al.: Internal kinematics of spiral galaxies in distant clusters, Online Material p 21
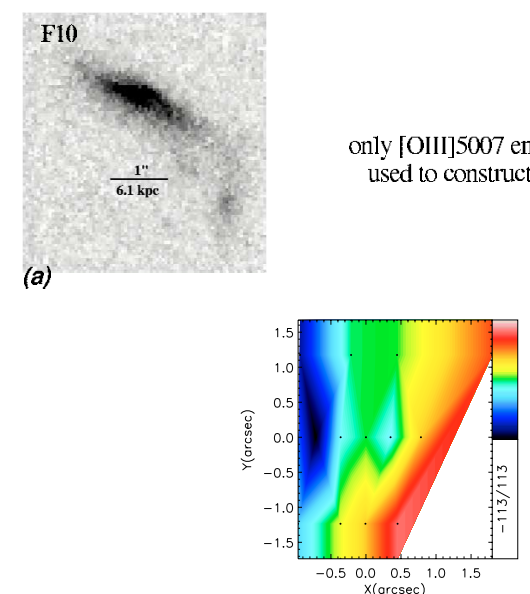

(e) $[0$ III $] 5007$ velocity field

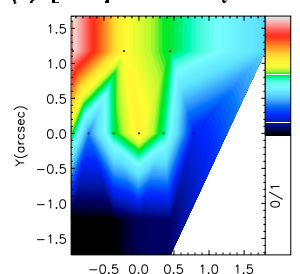

(h) [OIII] 50007 normalized flux map only [OIII]5007 emission line could be used to construct a velocity field

(a)

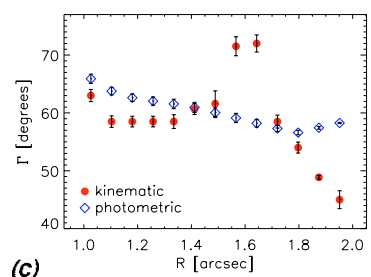

(c)

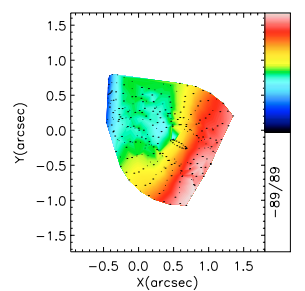

(f) reconstructed velocity map

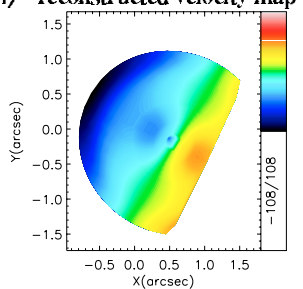

(i) VF for fixed $\Gamma$ and $q$
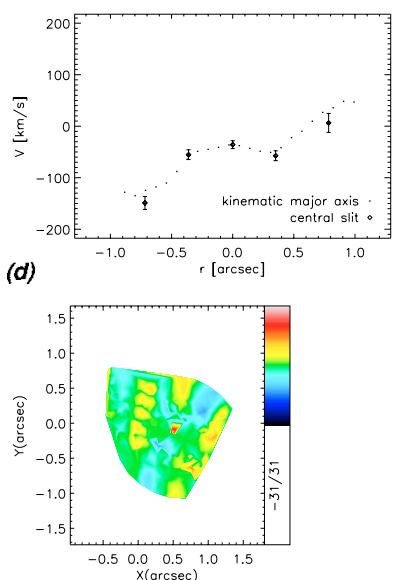

(g) residual of 't' and 'f'

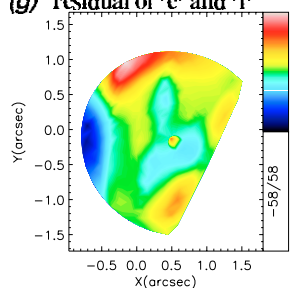

(i) residual of ' $\mathrm{e}$ ' and ' $\mathrm{i}$ '
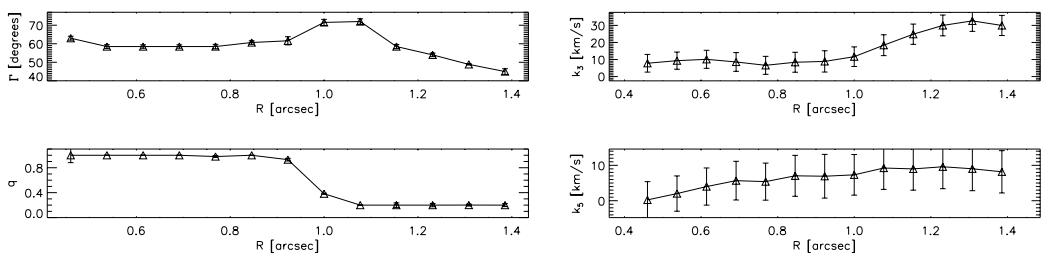

(k)

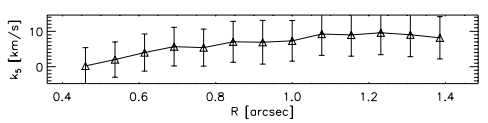

(I)

Fig. B.17. a) HST-ACS image of the galaxy in the $I$ band. c) Position angles of kinematic and photometric axes as a function of radius. d) Rotation curves extracted along the central slit and the kinematic major axis. e) [OIII]5007 velocity field. f) Velocity map reconstructed using 6 harmonic terms. g) Residual of the velocity map and the reconstructed map. h) Normalized [OIII]5007 flux map. i) Simple rotation map constructed for position angle and ellipticity fixed to their global values. j) Residual of the velocity map and the simple rotation map. k) Position angle and flattening as a function of radius. I) $k_{3} / k_{1}$ and $k_{5} / k_{1}$ (from the analysis where position angle and ellipticity are fixed to their global values) as a function of radius. 
E. Kutdemir et al.: Internal kinematics of spiral galaxies in distant clusters, Online Material p 22

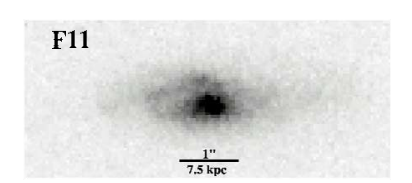

(a) only one cmission line is visible in the spectrum

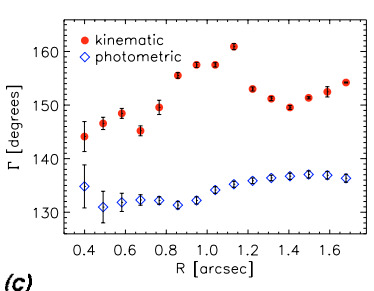

(c)

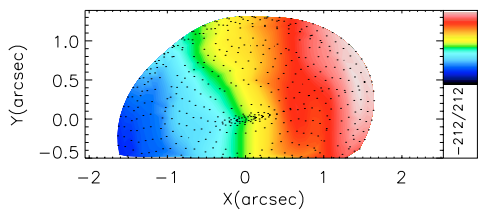

reconstructed velocity map

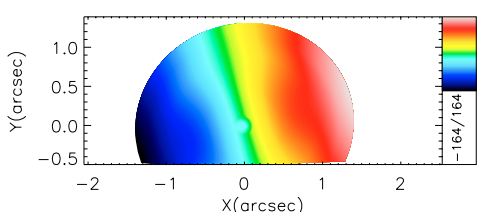

VF for fixed I' and $q$

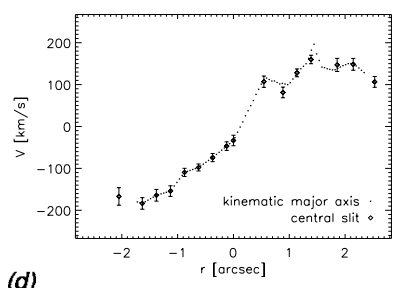

(d)

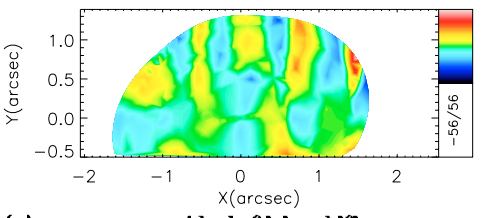

(g) residual of 'e' and ' $\mathrm{f}$

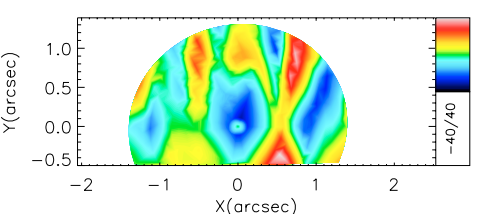

(j)
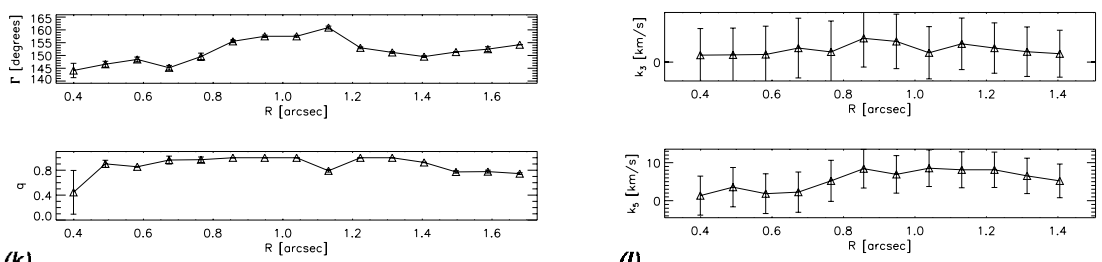

(l)

Fig. B.18. a) HST-ACS image of the galaxy in the $I$ band. c) Position angles of kinematic and photometric axes as a function of radius. d) Rotation curves extracted along the central slit and the kinematic major axis. e) Velocity field constructed using the emission line which could not be identified. f) Velocity map reconstructed using 6 harmonic terms. g) Residual of the velocity map and the reconstructed map. h) Normalized flux map of the emission line which could not be identified. i) Simple rotation map constructed for position angle and ellipticity fixed to their global values. j) Residual of the velocity map and the simple rotation map. k) Position angle and flattening as a function of radius. I) $k_{3} / k_{1}$ and $k_{5} / k_{1}$ (from the analysis where position angle and ellipticity are fixed to their global values) as a function of radius. 
E. Kutdemir et al.: Internal kinematics of spiral galaxies in distant clusters, Online Material p 23

\section{Appendix C: Photometric tables for the selected objects}

Table C.1. Photometric parameters for the MS 0451 sample.

\begin{tabular}{|c|c|c|c|c|c|c|c|c|c|}
\hline $\begin{array}{l}\text { ID } \\
\text { (1) }\end{array}$ & $\begin{array}{l}\text { RA } \\
\text { (2) }\end{array}$ & $\begin{array}{l}\text { Dec } \\
\text { (3) }\end{array}$ & $\begin{array}{c}z \\
(4)\end{array}$ & $\begin{array}{c}V \\
(5)\end{array}$ & $\begin{array}{c}R \\
(6)\end{array}$ & $\begin{array}{c}I \\
(7)\end{array}$ & $\begin{array}{l}M_{B} \\
\text { (8) }\end{array}$ & $\begin{array}{c}\text { A } \\
\text { (9) }\end{array}$ & $\begin{array}{c}\mathrm{C} \\
(10)\end{array}$ \\
\hline $\mathrm{C} 1$ & $04: 54: 2.2$ & $-02: 57: 10$ & 0.5421 & 21.94 & 20.85 & 19.95 & -21.06 & 0.25219 & 0.32179 \\
\hline $\mathrm{C} 2$ & $04: 54: 17.2$ & $-03: 01: 56$ & 0.5486 & 22.02 & 21.00 & 20.07 & -20.91 & 0.15682 & 0.34844 \\
\hline C3 & $04: 54: 18.6$ & $-03: 01: 03$ & 0.5465 & 22.16 & 21.20 & 20.49 & -20.68 & 0.07922 & 0.27806 \\
\hline $\mathrm{C} 4$ & $04: 54: 17.6$ & $-02: 59: 23$ & 0.5324 & 22.34 & 21.41 & 20.72 & -20.37 & 0.36244 & 0.33291 \\
\hline C5 & $04: 54: 1.5$ & $-02: 59: 24$ & 0.5312 & 22.42 & 21.01 & 19.81 & -20.82 & 0.15670 & 0.52285 \\
\hline C6 & $04: 54: 1.3$ & $-02: 59: 22$ & 0.5305 & 21.83 & 21.15 & 20.59 & -20.59 & 0.32947 & 0.40650 \\
\hline C7 & 04:54:5.0 & $-02: 59: 40$ & 0.5277 & 19.75 & 19.23 & 18.86 & -22.45 & 0.32994 & 0.20636 \\
\hline C8 & $04: 54: 4.4$ & $-03: 00: 14$ & 0.5325 & 20.80 & 20.10 & 19.46 & -21.68 & 0.27380 & 0.27789 \\
\hline C9 & $04: 54: 15.0$ & $-03: 00: 22$ & 0.5246 & 20.56 & 19.89 & 19.46 & -21.76 & 0.33459 & 0.26970 \\
\hline $\mathrm{C} 10$ & $04: 54: 17.7$ & $-03: 02: 29$ & 0.5312 & 21.92 & 21.61 & 21.21 & -20.05 & 0.19672 & 0.21096 \\
\hline $\mathrm{C} 11$ & $04: 54: 10.3$ & $-03: 00: 46$ & - & - & - & - & - & - & - \\
\hline F1 & $04: 54: 2.7$ & $-02: 58: 41$ & 0.9009 & 23.27 & 22.75 & 21.46 & -20.97 & 0.18516 & 0.18578 \\
\hline $\mathrm{F} 2$ & $04: 54: 3.8$ & $-02: 59: 19$ & 0.5795 & 20.88 & 20.11 & 19.42 & -21.96 & 0.27311 & 0.22909 \\
\hline F3 & $04: 54: 14.9$ & $-02: 58: 17$ & 0.5667 & 21.88 & 21.24 & 20.64 & -20.66 & 0.33834 & 0.20427 \\
\hline F4 & $04: 54: 7.8$ & $-03: 00: 33$ & 0.1867 & 20.54 & 20.37 & 20.00 & -18.78 & 0.17365 & 0.32308 \\
\hline F5 & $04: 54: 10.0$ & $-03: 00: 28$ & 0.1573 & 19.05 & 18.72 & 18.26 & -19.95 & 0.19844 & 0.30783 \\
\hline F6 & $04: 54: 18.9$ & $-03: 00: 05$ & 0.0982 & 19.23 & 18.93 & 18.55 & -18.56 & 0.14823 & 0.27114 \\
\hline F7 & $04: 54: 2.3$ & $-02: 58: 10$ & 0.9125 & 22.22 & 21.91 & 20.73 & -21.64 & 0.42715 & 0.16334 \\
\hline F8 & $04: 54: 10.4$ & $-03: 00: 47$ & 0.4443 & - & - & - & - & - & - \\
\hline F9 & $04: 54: 10.2$ & $-03: 01: 57$ & 0.3259 & 20.83 & 20.03 & 19.08 & -20.15 & 0.29565 & 0.53731 \\
\hline F10 & $04: 54: 0.5$ & $-02: 58: 15$ & 0.4947 & 22.47 & 21.86 & 21.38 & -19.67 & 0.20119 & 0.23420 \\
\hline F11 & $04: 54: 20.6$ & $-03: 00: 16$ & - & 21.25 & 20.67 & 20.04 & - & 0.18512 & 0.27344 \\
\hline
\end{tabular}

Column (1): object ID; Cols. (2, 3): RA and Dec (J2000); Col. (4): object redshift; Cols. (5-7): $V, R, I$ extinction corrected total magnitudes from the FORS2/VLT images; Col. (8): rest frame Johnson- $B$ magnitudes $k$-corrected with kcorrect (Blanton \& Roweis 2007); Col. (9): asymmetry index; Col. (10): concentration index.

For F11, the redshift is uncertain as explained in Sect. 2.3. Redshift of galaxy F7 and C11 are taken from Moran (2008). Galaxy C11 and F8 are so close together on the FORS images that their magnitudes could not be measured separately. 
E. Kutdemir et al.: Internal kinematics of spiral galaxies in distant clusters, Online Material p 24

Table C.2. Morphological parameters for the MS 0451 sample.

\begin{tabular}{|c|c|c|c|c|c|c|c|c|}
\hline $\begin{array}{l}\text { ID } \\
\text { (1) }\end{array}$ & $\begin{array}{l}\text { comp } \\
\text { (2) }\end{array}$ & $\begin{array}{c}F 814 W \\
\text { (3) }\end{array}$ & $\begin{array}{l}R_{\mathrm{e}} / R_{\mathrm{d}}(\mathrm{kpc}) \\
\quad(4)\end{array}$ & $\begin{array}{c}n \\
(5)\end{array}$ & $\begin{array}{c}q \\
(6)\end{array}$ & $\begin{array}{l}\text { PA } \\
\text { (7) }\end{array}$ & $\begin{array}{l}\chi^{2} \\
(8)\end{array}$ & $\begin{array}{l}\mathrm{B} / \mathrm{D} \\
(9)\end{array}$ \\
\hline $\mathrm{C} 1$ & exp disk & $20.04 \pm 0.01$ & $3.182 \pm 0.016$ & - & $0.31 \pm 0.00$ & $6.68 \pm 0.16$ & 1.95 & - \\
\hline \multirow[t]{2}{*}{$\mathrm{C} 2$} & exp disk & $20.35 \pm 0.01$ & $4.057 \pm 0.029$ & - & $0.68 \pm 0.00$ & $130.45 \pm 0.56$ & 1.62 & 0.20 \\
\hline & Sérsic bulge & $22.08 \pm 0.07$ & $0.840 \pm 0.071$ & $2.37 \pm 0.20$ & $0.99 \pm 0.02$ & - & - & \\
\hline C3 & exp disk & $20.44 \pm 0.00$ & $3.643 \pm 0.016$ & - & $0.23 \pm 0.00$ & $107.48 \pm 0.10$ & 1.89 & - \\
\hline $\mathrm{C} 4$ & exp disk & $20.67 \pm 0.00$ & $2.334 \pm 0.013$ & - & $0.16 \pm 0.00$ & $351.81 \pm 0.08$ & 2 & - \\
\hline \multirow[t]{2}{*}{$\mathrm{C} 5$} & exp disk & $21.33 \pm 0.03$ & $2.646 \pm 0.044$ & - & $0.19 \pm 0.00$ & $133.62 \pm 0.16$ & 1.93 & 2.05 \\
\hline & Sérsic bulge & $20.55 \pm 0.02$ & $1.619 \pm 0.035$ & $3.73 \pm 0.09$ & $0.47 \pm 0.01$ & - & - & \\
\hline C6 & exp disk & $21.08 \pm 0.00$ & $1.070 \pm 0.006$ & - & $0.90 \pm 0.01$ & $91.42 \pm 2.85$ & 2.56 & - \\
\hline $\mathrm{C} 7$ & exp disk & $18.87 \pm 0.00$ & $5.086 \pm 0.019$ & - & $0.78 \pm 0.00$ & $104.2 \pm 0.51$ & 2.72 & - \\
\hline \multirow[t]{2}{*}{$\mathrm{C} 8$} & exp disk & $19.62 \pm 0.00$ & $4.983 \pm 0.025$ & - & $0.64 \pm 0.00$ & $116.19 \pm 0.40$ & 2.24 & 0.05 \\
\hline & Sérsic bulge & $22.86 \pm 0.02$ & $0.419 \pm 0.016$ & $1.43 \pm 0.19$ & $0.74 \pm 0.03$ & - & - & \\
\hline C9 & exp disk & $19.48 \pm 0.00$ & $3.693 \pm 0.016$ & - & $0.50 \pm 0.00$ & $110.27 \pm 0.23$ & 3.56 & - \\
\hline $\mathrm{C} 10$ & exp disk & $21.62 \pm 0.01$ & $1.071 \pm 0.009$ & - & $0.80 \pm 0.01$ & $30.22 \pm 2.13$ & 2.65 & - \\
\hline $\mathrm{F} 1$ & exp disk & $21.43 \pm 0.01$ & $3.351 \pm 0.039$ & - & $0.51 \pm 0.00$ & $19.77 \pm 0.58$ & 1.54 & - \\
\hline F2 & exp disk & $19.41 \pm 0.00$ & $3.880 \pm 0.016$ & - & $0.90 \pm 0.00$ & $137.13 \pm 1.32$ & 2.06 & - \\
\hline F3 & exp disk & $20.66 \pm 0.01$ & $4.489 \pm 0.026$ & - & $0.22 \pm 0.00$ & $123.95 \pm 0.12$ & 1.61 & - \\
\hline F4 & exp disk & $20.08 \pm 0.00$ & $2.062 \pm 0.011$ & - & $0.33 \pm 0.00$ & $96.37 \pm 0.15$ & 2.13 & - \\
\hline \multirow[t]{2}{*}{ F5 } & exp disk & $18.28 \pm 0.00$ & $3.453 \pm 0.007$ & - & $0.36 \pm 0.00$ & $129.11 \pm 0.07$ & 2.41 & 0.05 \\
\hline & Sérsic bulge & $21.64 \pm 0.03$ & $0.400 \pm 0.018$ & $1.84 \pm 0.11$ & $0.89 \pm 0.02$ & - & - & \\
\hline \multirow[t]{2}{*}{ F6 } & exp disk & $18.47 \pm 0.00$ & $2.432 \pm 0.012$ & - & $0.65 \pm 0.00$ & $179.96 \pm 0.24$ & 2.19 & 0.04 \\
\hline & Sérsic bulge & $21.88 \pm 0.07$ & $0.500 \pm 0.039$ & $1.62 \pm 0.12$ & $1.00 \pm 0.03$ & - & - & \\
\hline F7 & exp disk & $20.96 \pm 0.01$ & $3.175 \pm 0.031$ & - & $0.71 \pm 0.01$ & $47.28 \pm 0.93$ & 1.73 & - \\
\hline \multirow[t]{2}{*}{ F9 } & exp disk & $20.36 \pm 0.02$ & $2.451 \pm 0.021$ & - & $0.29 \pm 0.00$ & $9.36 \pm 0.18$ & 2.05 & 1.51 \\
\hline & Sérsic bulge & $19.91 \pm 0.02$ & $1.602 \pm 0.033$ & $3.09 \pm 0.05$ & $0.60 \pm 0.00$ & - & - & \\
\hline F10 & exp disk & $21.33 \pm 0.01$ & $3.581 \pm 0.039$ & - & $0.28 \pm 0.00$ & $61.08 \pm 0.26$ & 1.81 & - \\
\hline \multirow[t]{2}{*}{ F11 } & exp disk & $20.14 \pm 0.01$ & $0.7 \pm 0.007$ & - & $0.37 \pm 0.00$ & $134.39 \pm 0.18$ & 1.92 & 0.12 \\
\hline & Sérsic bulge & $22.44 \pm 0.12$ & $0.245 \pm 0.025$ & $1.33 \pm 0.10$ & $0.85 \pm 0.03$ & - & - & \\
\hline
\end{tabular}

Column (1): object ID; Col. (2): component; Col. (3): total magnitude; Col. (4): effective radius of the bulge/scale length of the disk; Col. (5): sérsic index of the bulge profile; Col. (6): flattening; Col. (7): position angle of the disk measured from North through East; Col. (8): $\chi^{2}$ of the fit; Col. (9): bulge to disk ratio.

The photometric zero point of the magnitude measurements is $Z_{\mathrm{p}}^{F 814 W}=25.492$ (Bedin et al. 2005). The redshift of galaxy F11 is uncertain as explained in Sect. 2.3. As a result, for this galaxy, Col. (4) is given in arcseconds. For galaxy F7, we used the redshift from Moran (2008). The surface brightness profile of Galaxy C10 is much more extended on one side of the galaxy than on the other side. Therefore the scale length and total magnitude of the disk given in the table are not reliable. This galaxy was excluded while calculating the correlations between the disk scale length and the irregularity in the gas kinematics. 